\title{
ISOPARAMETRIC MIXED FINITE ELEMENT APPROXIMATION OF EIGENVALUES AND EIGENVECTORS OF 4TH ORDER EIGENVALUE PROBLEMS WITH VARIABLE COEFFICIENTS
}

\author{
Pulin Kumar Bhattacharyya ${ }^{1}$ And NeEla Nataraj ${ }^{2}$
}

\begin{abstract}
Estimates for the combined effect of boundary approximation and numerical integration on the approximation of (simple) eigenvalues and eigenvectors of 4 th order eigenvalue problems with variable/constant coefficients in convex domains with curved boundary by an isoparametric mixed finite element method, which, in the particular case of bending problems of aniso-/ortho-/isotropic plates with variable/constant thickness, gives a simultaneous approximation to bending moment tensor field $\Psi=\left(\psi_{i j}\right)_{1 \leq i, j \leq 2}$ and displacement field ' $\mathrm{u}$ ', have been developed.
\end{abstract}

Mathematics Subject Classification. 35J40, 65N30, 35P99, 74H45.

Received: May 5, 2001.

\section{INTRODUCTION}

In all papers $[14,22,23,29]$ on mixed finite element analysis of 4 th order eigenvalue problems, it has been assumed that neither any numerical integration is essential nor any approximation of the boundary is necessary (since the boundary of the convex domain is a polygonal one in all the cases, the convexity of the domain being a requirement for the regularity of the solution $[18,21,24]$ ). But in many situations, we are to consider convex domains with curved boundary $\Gamma$. Then an approximation of the curved boundary and possibly numerical evaluation of integrals will be essential, but convergence analysis becomes much more complex and complicated. Even for classical, standard finite element analysis of second order self-adjoint eigenvalue problems in domains with curved boundary we find the situation as stated in ( [40], p. 254): “.. In contrast to finite element analysis of boundary value problems, in the finite element analysis of eigenvalue problems, there does not exist any abstract error estimate consisting of the sum of three terms (error of interpolation, error of approximation of the boundary and error of numerical integration) ...". Hence, in such a situation error analysis for each specific problem can be attempted at and the proofs involved in finding the estimates will be quite complex and too technical in nature due to these additional complicacies introduced by the boundary approximation and obligatory use (for example, in the isoparametric case) of numerical integration. In fact, we find only two papers $[25,40]$, in which this combined effect of boundary approximation and numerical integration on

Keywords and phrases. Mixed FEM, eigenvalue problem, isoparametric boundary approximation, 4th-order equations, anisotropic plates, convergence analysis, numerical results.

1 Visiting Professor, School of Computer and Systems Sciences, Jawaharlal Nehru University, New Delhi: 110067, India.

e-mail: pulinkum@hotmail.com

2 Lecturer, Department of Mathematics, Indian Institute of Technology, New Delhi, 110016, India.

e-mail: neela@maths.iitd.ernet.in 
second order self-adjoint eigenvalue approximations using classical isoparametric finite element methods has been estimated, [5] and [6] being the papers which deal with the effect of only numerical integration on eigenvalue approximations. But the situation is still worse in the case of isoparametric mixed finite element analysis of eigenvalue problems, for which error estimates are to be developed again for a specific mixed method formulation (since abstract results for the isoparametric case do not exist even for source problems) and the proofs for the estimates will be much more complex and much more technical in nature. Indeed to our knowledge, [8] is probably the first publication on the estimates for the combined effect of boundary approximation and numerical integration on the mixed finite element approximation of (simple) eigenvalues and eigenvectors of 4th order self-adjoint eigenvalue problems with variable/constant coefficients, many proofs in which, as stated earlier, have remained quite technical in spite of the best efforts of the authors to avoid these technical aspects in some proofs. The present paper, the results of which were announced in [8] (see also [31]), relies heavily on [10] for the corresponding source problem ([9] contains error estimates due to polygonal approximation of the curved boundary along with numerical integration for the same source problem) and also on the results of [4] on the mixed method scheme (see also $[33,36]$ ) for polygonal domains. For other interesting references on eigenvalue approximations, we refer to $[2,15]$. Finally, the present paper also contains interesting results of numerical experiments on some problems of practical importance and research interest.

\section{The CONTINuOUs miXed VARIATIONAL EIGENVALUe PROBlem}

Consider the eigenvalue problem: Find $\lambda \in \mathbb{R}$ for which $\exists$ non-null $\mathrm{u}$ such that

$$
\left(\mathbf{P}^{\mathbf{E}}\right): \quad \Lambda u=\lambda u \text { in } \Omega,\left.u\right|_{\Gamma}=\left.\left(\frac{\partial u}{\partial n}\right)\right|_{\Gamma}=0,
$$

where $(\Lambda u)(x) \equiv \sum_{i=1}^{2} \sum_{j=1}^{2} \sum_{k=1}^{2} \sum_{l=1}^{2} \frac{\partial^{2}}{\partial x_{k} \partial x_{l}}\left(a_{i j k l} \frac{\partial^{2} u}{\partial x_{i} \partial x_{j}}\right)(x) \equiv\left(a_{i j k l} u_{, i j}\right)_{, k l}(x) \quad \forall x=\left(x_{1}, x_{2}\right) \in \Omega$.

(In (2.2) and also in the sequel, Einstein's summation convention with respect to twice repeated indices $i, j, k, l=1,2$ has been followed unless stated otherwise).

(A1): $\Omega$ is a bounded, open, convex domain with Lipschitz continuous boundary $\Gamma$ which is piecewise of $C^{k}$ - class, $k \geq 3[1,21,35,41]$;

coefficients $a_{i j k l}=a_{i j k l}(x) \forall x=\left(x_{1}, x_{2}\right) \in \overline{\widetilde{\Omega}} \subset \mathbb{R}^{2}$,

(A2): $\widetilde{\Omega}$ being a bounded, open set with boundary $\widetilde{\Gamma}$, which is piecewise of $C^{k}$-class, $k \geq 3$, such that $\bar{\Omega}=\Omega \cup \Gamma \subset \widetilde{\Omega}$

satisfy the following assumptions: $\forall i, j, k, l=1,2$,

$(\mathbf{A 3}): a_{i j k l} \in W^{2, \infty}(\widetilde{\Omega}) \hookrightarrow C^{1}(\overline{\widetilde{\Omega}}) ; \quad a_{i j k l} \geq 0 ; a_{i j k l}(x)=a_{k l i j}(x)=a_{l k i j}(x)=a_{l k j i}(x) \forall x \in \overline{\widetilde{\Omega}} ;$

(A4): $\exists \alpha>0$ such that $\forall \underline{\xi}=\left(\xi_{11}, \xi_{12}, \xi_{21}, \xi_{22}\right) \in \mathbb{R}^{4}$ with $\xi_{21}=\xi_{12}, a_{i j k l}(x) \xi_{i j} \xi_{k l} \geq \alpha\|\underline{\xi}\|_{\mathbb{R}^{4}}^{2} \forall x \in \overline{\widetilde{\Omega}}$.

Then, the corresponding Galerkin Variational Eigenvalue Problem $\left(\mathbf{P}_{\mathbf{G}}^{\mathbf{E}}\right)$ is defined by: Find $\lambda \in \mathbb{R}$ for which $\exists$ non-null $u \in H_{0}^{2}(\Omega)[1,28]$ such that

$$
\left(\mathbf{P}_{\mathbf{G}}^{\mathbf{E}}\right): a(u, v)=\lambda\langle u, v\rangle_{0, \Omega} \quad \forall v \in H_{0}^{2}(\Omega)
$$


where $a(u, v)=\langle\Lambda u, v\rangle_{0, \Omega}=\int_{\Omega} a_{i j k l} u_{, i j} v_{, k l} \mathrm{~d} \Omega=a(v, u) \forall u, v \in H_{0}^{2}(\Omega)$;

$\exists \alpha_{0}>0$ such that $a(v, v) \geq \alpha_{0}\|v\|_{2, \Omega}^{2} \forall v \in H_{0}^{2}(\Omega) \quad[3,20] \quad\langle u, v\rangle_{0, \Omega}=\int_{\Omega} u v \mathrm{~d} x$.

Since $a(\cdot, \cdot)$ is continuous and $H_{0}^{2}(\Omega)$-elliptic, the associated Galerkin Variational (Source) Problem $\left(\mathbf{P}_{\mathbf{G}}\right)$ defined by: For given $f \in L^{2}(\Omega)$, find $u \in H_{0}^{2}(\Omega)$ such that

$$
\left(\mathbf{P}_{\mathbf{G}}\right): a(u, v)=\langle f, v\rangle_{0, \Omega} \quad \forall v \in H_{0}^{2}(\Omega),
$$

has a unique solution by Lax-Milgram lemma, and we have:

Theorem 2.1 ( [8]). ( $\left.\mathbf{P}_{\mathbf{G}}^{\mathbf{E}}\right)$ has a countable non-decreasing system of strictly positive eigenvalues with possibly finite multiplicities and accumulation point at $\infty$ : i.e. $0<\widehat{\lambda}_{1} \leq \widehat{\lambda}_{2} \leq \cdots \leq \widehat{\lambda}_{m} \leq \cdots \uparrow \infty$, and $\exists$ a system of eigenpairs $\left(\widehat{\lambda}_{m}, \widehat{v}_{m}\right)_{m=1}^{\infty}$ such that the eigensystem $\left(\widehat{v}_{m}\right)_{m=1}^{\infty}$ is a Hilbert basis in $\left(H_{0}^{2}(\Omega) ;\langle\langle\cdot, \cdot\rangle\rangle_{a(\cdot, \cdot)}\right)$ with $\left\langle\left\langle\widehat{v}_{m}, \widehat{v}_{n}\right\rangle\right\rangle_{a(\cdot, \cdot)}=a\left(\widehat{v}_{m}, \widehat{v}_{n}\right)=\delta_{m n}$. Moreover, $\left(\sqrt{\widehat{\lambda}_{m}} \widehat{v}_{m}\right)_{m=1}^{\infty}$ is a Hilbert basis in $L^{2}(\Omega)$.

Now, defining Hilbert space $\mathbf{H}$ of symmetric tensor-valued functions in $\Omega$ by:

$$
\mathbf{H}=\left\{\Phi: \Phi=\left(\phi_{i j}\right)_{1 \leq i, j \leq 2} \text { with } \phi_{i j}=\phi_{j i} \in L^{2}(\Omega)\right\} \text { with }\|\Phi\|_{\mathbf{H}}^{2}=\|\Phi\|_{0, \Omega}^{2}=\sum_{i, j=1}^{2} \int_{\Omega}\left|\phi_{i j}(x)\right|^{2} \mathrm{~d} x
$$

and new coefficients $A_{i j k l}=A_{i j k l}(x) \quad \forall x \in \overline{\widetilde{\Omega}}$ in terms of coefficients $a_{i j k l}$, the algorithm for which is given in [4], satisfying the following properties: $\forall i, j, k, l=1,2$

$$
\bullet \forall x \in \overline{\widetilde{\Omega}}, \quad A_{i j k l}(x)=A_{k l i j}(x)=A_{l k i j}(x)=A_{l k j i}(x)
$$

$\bullet \exists \alpha>0$ such that $A_{i j k l}(x) \xi_{i j} \xi_{k l} \geq \alpha\|\underline{\xi}\|_{\mathbb{R}^{4}}^{2} \forall x \in \widetilde{\widetilde{\Omega}}, \forall \underline{\xi}=\left(\xi_{i j}\right)_{i, j=1,2} \in \mathbb{R}^{4}$ with $\xi_{12}=\xi_{21} ;$

- $\forall x \in \overline{\widetilde{\Omega}}, \quad \forall \underline{\xi}=\left(\xi_{i j}\right)_{i, j=1,2} \in \mathbb{R}^{4}$ with $\xi_{21}=\xi_{12}, \forall \underline{\zeta}=\left(\zeta_{i j}\right)_{i, j=1,2} \in \mathbb{R}^{4}$ with $\zeta_{21}=\zeta_{12}$,

$$
A_{i j k l}(x) a_{i j m n}(x) \xi_{m n} \zeta_{k l}=\xi_{i j} \zeta_{i j} ; A_{i j k l}(x) a_{i j m n}(x) \xi_{m n}=\xi_{k l} \text {, and }
$$

(A5): $A_{i j k l} \in W^{2, \infty}(\widetilde{\Omega}) \hookrightarrow C^{1}(\overline{\widetilde{\Omega}})$,

we construct an Auxiliary Continuous Mixed Variational Eigenvalue Problem $\left(\mathbf{Q}_{\mathbf{A}}^{\mathbf{E}} \mathbf{x}\right)$ as follows: Find $\lambda \in \mathbb{R}$ for which $\exists$ non-null $(\Psi, u) \in \mathbf{H} \times H_{0}^{2}(\Omega)$ (i.e. $\left.\Psi \neq \mathbf{0}, u \neq 0\right)$ such that

$$
\left(\mathbf{Q}_{\mathbf{A U X}}^{\mathbf{E}}\right): A_{0}(\Psi, \Phi)+b_{0}(\Phi, u)=0 \quad \forall \Phi \in \mathbf{H} ; \quad-b_{0}(\Psi, v)=\lambda\langle u, v\rangle_{0, \Omega} \quad \forall v \in H_{0}^{2}(\Omega) .
$$

The associated Source Problem (Q $\mathbf{Q}_{\text {AUX }}$ ) in Continuous Mixed Variational Formulation is defined by: For given $f \in L^{2}(\Omega)$, find $(\Psi, u) \in \mathbf{H} \times H_{0}^{2}(\Omega)$ such that:

$$
\left(\mathbf{Q}_{\mathbf{A U X}}\right): A_{0}(\Psi, \Phi)+b_{0}(\Phi, u)=0 \quad \forall \Phi \in \mathbf{H} ; \quad-b_{0}(\Psi, v)=\langle f, v\rangle_{0, \Omega} \quad \forall v \in H_{0}^{2}(\Omega),
$$

where $A_{0}(\cdot, \cdot)$ and $b_{0}(\cdot, \cdot)$ are continuous bilinear forms defined by:

$$
A_{0}(\Psi, \Phi)=\int_{\Omega} A_{i j k l} \psi_{i j} \phi_{k l} \mathrm{~d} x=A_{0}(\Phi, \Psi) \text { with }\left|A_{0}(\Psi, \Phi)\right| \leq \bar{M}_{0}\|\Psi\|_{\mathbf{H}}\|\Phi\|_{\mathbf{H}} \text { for some } \bar{M}_{0}>0
$$




$$
A_{0}(\Phi, \Phi) \geq \alpha\|\Phi\|_{\mathbf{H}}^{2} \forall \Phi \in \mathbf{H} \text { and for some } \alpha>0 ;
$$

$$
\begin{gathered}
b_{0}(\Phi, v)=-\int_{\Omega} \phi_{i j} v_{, i j} \mathrm{~d} \Omega \forall \Phi \in \mathbf{H} \forall v \in H_{0}^{2}(\Omega) \text { with }\left|b_{0}(\Phi, v)\right| \leq \bar{m}_{0}\|\Phi\|_{\mathbf{H}}\|v\|_{2, \Omega} \text { for some } \bar{m}_{0}>0, \\
\sup _{\Phi \in \mathbf{H}-\{0\}} \frac{\left|b_{0}(\Phi, v)\right|}{\|\Phi\|_{\mathbf{H}}} \geq \beta_{0}\|v\|_{2, \Omega} \forall v \in H_{0}^{2}(\Omega) \text { for some } \beta_{0}>0 .
\end{gathered}
$$

As a consequence of (2.10) and (2.11), ( $\left.\mathbf{Q}_{\mathbf{A U X}}\right)$ has a unique solution $(\Psi, u) \in \mathbf{H} \times H_{0}^{2}(\Omega)[2,11,12]$, and we define $\overrightarrow{\mathbf{T}}_{\mathbf{0}}: f \in L^{2}(\Omega) \mapsto \overrightarrow{\mathbf{T}}_{\mathbf{0}} f=\left(S_{0} f, T_{0} f\right)=(\Psi, u) \in \mathbf{H} \times H_{0}^{2}(\Omega)$ such that

$$
A_{0}\left(S_{0} f, \Phi\right)+b_{0}\left(\Phi, T_{0} f\right)=0 \quad \forall \Phi \in \mathbf{H} ; \quad-b_{0}\left(S_{0} f, v\right)=\langle f, v\rangle_{0, \Omega} \quad \forall v \in H_{0}^{2}(\Omega),
$$

where $S_{0} \in \mathcal{L}\left(L^{2}(\Omega) ; \mathbf{H}\right), T_{0} \in \mathcal{L}\left(L^{2}(\Omega) ; H_{0}^{2}(\Omega)\right)$ with

$$
\left\|S_{0} f\right\|_{0, \Omega}+\left\|T_{0} f\right\|_{2, \Omega} \leq C\|f\|_{0, \Omega} \quad \forall f \in L^{2}(\Omega), S_{0} f=\Psi, T_{0} f=u ; S_{0}(\cdot)=\left(\left(a_{i j k l} T_{0}(\cdot)_{, k l}\right)_{1 \leq i, j \leq 2} .\right.
$$

Then, $\hookrightarrow \cdot T_{0}=T_{0} \in \mathcal{L}\left(L^{2}(\Omega) ; L^{2}(\Omega)\right) \equiv \mathcal{L}\left(L^{2}(\Omega)\right)$ with $\hookrightarrow: H_{0}^{2}(\Omega) \longrightarrow L^{2}(\Omega)$ is a compact, positive, symmetric, linear operator and the eigenvalue problem of $T_{0} \in \mathcal{L}\left(L^{2}(\Omega)\right): T_{0} u=\mu u$ is "equivalent" to the eigenvalue problem $\left(\mathbf{Q}_{\mathbf{A} \mathbf{U} \mathbf{X}}^{\mathbf{E}}\right)$ with $\mu=1 / \lambda>0$. Hence, we have:

Theorem 2.2. ( $\mathbf{Q}_{\mathrm{AUX}}^{\mathrm{E}}$ ) has a countable system of strictly positive, non-decreasing system of eigenvalues with possibly finite multiplicities and accumulation point at $\infty$ :

$$
0<\lambda_{1} \leq \lambda_{2} \leq \cdots \leq \lambda_{m} \leq \cdots \uparrow \infty \text { with } \mu_{m}=1 / \lambda_{m} \forall m \in \mathbb{N},
$$

and $\exists$ eigenpairs $\left(\lambda_{m} ;\left(\Psi_{m}, u_{m}\right)\right)_{m=1}^{\infty}$ of $\left(\mathbf{Q}_{\mathbf{A U X}}^{\mathbf{E}}\right): \forall m \in \mathbb{N}$,

$$
A_{0}\left(\Psi_{m}, \Phi\right)+b_{0}\left(\Phi, u_{m}\right)=0 \quad \forall \Phi \in \mathbf{H} ; \quad-b_{0}\left(\Psi_{m}, v\right)=\lambda_{m}\left\langle u_{m}, v\right\rangle_{0, \Omega} \forall v \in H_{0}^{2}(\Omega),
$$

$\left(u_{m}\right)_{m=1}^{\infty}$ being a Hilbert basis in $L^{2}(\Omega)$ with $\Psi_{m}=S_{0}\left(\lambda_{m} u_{m}\right)=\left(a_{i j k l} u_{m, k l}\right)_{i, j=1,2} \forall m \in \mathbb{N}$ (see (2.13)). Moreover, $\left(\frac{\Psi_{m}}{\sqrt{\lambda_{m}}}\right)_{m=1}^{\infty}$ is an orthonormal system in $\left(\mathbf{H},[\cdot, \cdot]_{A_{0}(\cdot, \cdot)}\right)$ with $\left[\frac{\Psi_{m}}{\sqrt{\lambda_{m}}}, \frac{\Psi_{n}}{\sqrt{\lambda_{n}}}\right]_{A_{0}(\cdot, \cdot)}=A_{0}\left(\frac{\Psi_{m}}{\sqrt{\lambda_{m}}}, \frac{\Psi_{n}}{\sqrt{\lambda_{n}}}\right)=\delta_{m n} \quad \forall m, n \in \mathbb{N}$.

As a consequence of $(2.10)$ and (2.11), $\forall$ fixed $v \in H_{0}^{2}(\Omega)$, there exists a unique $\underline{\sigma} \in \mathbf{H}$ such that $A_{0}(\underline{\sigma}, \Phi)+$ $b_{0}(\Phi, v)=0 \quad \forall \Phi \in \mathbf{H}$ by virtue of Lax-Milgram lemma and this correspondence defines $\mathcal{I}: v \in H_{0}^{2}(\Omega) \mapsto \mathcal{I} v=$ $\underline{\sigma} \in \mathbf{H}$ and we set

$$
\mathcal{E}=\mathcal{I}\left(H_{0}^{2}(\Omega)\right)=\left\{\underline{\sigma}: \underline{\sigma} \in \mathbf{H} \text { for which } \exists v \in H_{0}^{2}(\Omega) \text { such that } \mathcal{I} v=\underline{\sigma}\right\} \subset \mathbf{H} .
$$

Proposition 2.1. (i) $\left(\mathcal{E} ;[\cdot, \cdot]_{A_{0}(\cdot, \cdot)}\right)$ equipped with inner product $[\underline{\sigma}, \underline{\omega}]_{A_{0}(\cdot, \cdot)}=A_{0}(\underline{\sigma}, \underline{\omega}) \forall \underline{\sigma}, \underline{\omega} \in \mathcal{E}$ is a Hilbert space and $\forall$ eigenpair $\left(\lambda_{m} ;\left(\Psi_{m}, u_{m}\right)\right)$ of $\left(\mathbf{Q}_{\mathbf{A U X}}^{\mathbf{E}}\right), \Psi_{m}=\mathcal{I} u_{m}=S_{0}\left(\lambda_{m} u_{m}\right), m \in \mathbb{N}$.

(ii) $\mathcal{I}:\left(H_{0}^{2}(\Omega),\langle\langle\cdot, \cdot\rangle\rangle_{a(\cdot, \cdot)}\right) \longrightarrow\left(\mathcal{E} ;[\cdot, \cdot]_{A_{0}(\cdot, \cdot)}\right)$ is a linear, continuous bijection with

$\langle\langle v, w\rangle\rangle_{a(\cdot, \cdot)}=[\mathcal{I} v, \mathcal{I} w]_{A_{0}(\cdot, \cdot)}=[\underline{\sigma}, \underline{\omega}]_{A_{0}(\cdot, \cdot)}=A_{0}(\underline{\sigma}, \underline{\omega}),(\underline{\sigma}, v),(\underline{\omega}, w)$ being the linked pairs in $\mathcal{E} \times H_{0}^{2}(\Omega)$.

Set $\mathcal{M}=\mathcal{E} \times H_{0}^{2}(\Omega)=$ product space of linked pairs $(\underline{\sigma}, v)$ with $\underline{\sigma}=\mathcal{I} v \quad \forall v \in H_{0}^{2}(\Omega)$ 


\section{Rayleigh quotient characterization of eigenpairs.}

To $\left(\mathbf{P}_{\mathbf{G}}^{\mathbf{E}}\right)$ we can associate Rayleigh coefficient $R(v)=\frac{a(v, v)}{\langle v, v\rangle_{0, \Omega}} \forall v \in H^{2}(\Omega)-\{0\}$.

But $a(v, v)=\langle\langle v, v\rangle\rangle_{a(\cdot, \cdot)}=A_{0}(\underline{\sigma}, \underline{\sigma})$ with $\underline{\sigma}=\mathcal{I} v \in \mathcal{E}$. So $\forall v \in H_{0}^{2}(\Omega)$ with $\underline{\sigma}=\mathcal{I} v \in \mathcal{E}, R(v)=\frac{A_{0}(\underline{\sigma}, \underline{\sigma})}{\langle v, v\rangle_{0, \Omega}}$. i.e. $R(v)$ is expressed through a linked pair $(\underline{\sigma}, v)=(\mathcal{I} v, v) \in \mathcal{E} \times H_{0}^{2}(\Omega)$. Hence, it suggests to define a new Rayleigh quotient $\Re(\cdot, \cdot)$ on $\mathcal{M} \equiv \mathcal{E} \times H_{0}^{2}(\Omega)$ by (see also [14]): $\Re(\underline{\sigma}, v)=\frac{A_{0}(\underline{\sigma}, \underline{\sigma})}{\langle v, v\rangle_{0, \Omega}} \quad \forall$ linked pair $(\underline{\sigma}, v)=(\mathcal{I} v, v) \in \mathcal{M}$ such that $\Re(\underline{\sigma}, v) \equiv R(v)=\frac{a(v, v)}{\langle v, v\rangle_{0, \Omega}} \forall v \in H_{0}^{2}(\Omega)-\{0\}$, for which we can apply various extrema $[2,14,35,37]$.

Define a $p$-dimensional subspace $\mathcal{M}_{p}$ (resp. $\left.U_{p}\right)$ of $\mathcal{M}\left(\right.$ resp. $\left.H_{0}^{2}(\Omega)\right)$ by: $\mathcal{M}_{p}=\operatorname{Span}\left\{\left(\Psi_{m}, u_{m}\right)_{m=1}^{p}\right\}$; $U_{p}=\operatorname{Span}\left\{\left(u_{m}\right)_{m=1}^{p}\right\}, \quad\left(\lambda_{m} ;\left(\Psi_{m}, u_{m}\right)\right) \in \mathbb{R}^{+} \times\left(\mathcal{E} \times H_{0}^{2}(\Omega)\right), \quad 1 \leq m \leq p$, being the first ' $p$ ' eigenpairs of $\left(\mathbf{Q}_{\text {AUX }}^{\mathrm{E}}\right)$ with $0<\lambda_{1} \leq \lambda_{2} \leq \cdots \leq \lambda_{p}$.

Theorem 2.3 (Min-Max Principle, $[2,37]$ ).

(i) Eigensolutions of $\left(\mathbf{Q}_{\mathbf{A U X}}^{\mathbf{E}}\right)$ are the stationary points of $\Re(\cdot, \cdot)$ on $\mathcal{M}$, the corresponding eigenvalues being the values of $\Re(\cdot, \cdot)$ at these stationary points;

(ii) $\forall p \in \mathbb{N}, \quad \lambda_{p}=\min _{\substack{\mathcal{S}_{p} \subset \mathcal{M} \\ \operatorname{dim}\left(\mathcal{S}_{p}\right)=p}} \max _{(\underline{\sigma}, v) \in \mathcal{S}_{p}} \Re(\underline{\sigma}, v)=\max _{(\underline{\sigma}, v) \in \mathcal{M}_{p}} \Re(\underline{\sigma}, v)=\Re\left(\Psi_{p}, u_{p}\right)$.

We will need another Rayleigh quotient $Q(v)=\frac{\left\langle T_{0} v, v\right\rangle_{0, \Omega}}{\langle v, v\rangle_{0, \Omega}} \quad \forall v \in H_{0}^{2}(\Omega)$, where $T_{0} \in \mathcal{L}\left(L^{2}(\Omega)\right)$ is compact, positive and symmetric. Hence,

Theorem 2.4 (Max-Min Principle, $[2,37]) . \forall p \in \mathbb{N}$,

$$
\mu_{p}=\max _{\substack{S_{p}^{*} \subset L^{2}(\Omega) \\ \operatorname{dim} S_{p}^{*}=p}} \min _{v \in S_{p}^{*}} Q(v)=\min _{\substack{v \in U_{p} \\ U_{p}=\operatorname{Span}\left\{\left(u_{m}\right)_{m=1}^{p}\right\}}} Q(v)=Q\left(u_{p}\right),
$$

where $\left(\mu_{m}, u_{m}\right)_{m=1}^{p}$ are the first ' $p$ ' eigenpairs of $T_{0}$ corresponding to the first ' $p$ ' eigenvalues $\mu_{1} \geq \mu_{2} \geq \cdots \geq \mu_{p}$ of $T_{0}, u_{m}$ being the $m$-th eigenvector of $T_{0}\left(\mu_{m}=1 / \lambda_{m}, 1 \leq m \leq p\right)$.

But $\left(\mathbf{Q}_{\mathbf{A U X}}^{\mathbf{E}}\right)$ is not suitable for finite element approximation, since $C^{1}$-elements are to be used for construction of finite element subspaces of $H_{0}^{2}(\Omega)$. Hence, we construct a new Continuous Mixed Variational Eigenvalue Problem $\left(\mathbf{Q}^{\mathbf{E}}\right)$, which will be eminently suitable for finite element approximation using $C^{0}$-elements as follows:

Find $\lambda \in \mathbb{R}$ for which $\exists$ non-null $(\Psi, u) \in \mathbf{V} \times W$ such that

$$
\left(\mathbf{Q}^{\mathbf{E}}\right): A(\Psi, \Phi)+b(\Phi, u)=0 \quad \forall \Phi \in \mathbf{V}, \quad-b(\Psi, v)=\lambda\langle u, v\rangle_{0, \Omega} \quad \forall v \in W,
$$

where $\mathbf{V}=\left\{\Phi: \Phi=\left(\phi_{i j}\right)_{i, j=1,2} \in \mathbf{H}, \phi_{i j} \in H^{1}(\Omega) \forall i, j=1,2\right\}$ with $\|\Phi\|_{\mathbf{V}}^{2}=\|\Phi\|_{1, \Omega}^{2}=\sum_{i=1}^{2} \sum_{j=1}^{2}\left\|\phi_{i j}\right\|_{1, \Omega}^{2}$ $W \equiv H_{0}^{1}(\Omega)=\left\{v: v \in H^{1}(\Omega),\left.v\right|_{\Gamma}=0\right\}$ with $\|v\|_{W}=\|v\|_{1, \Omega}$;

$A(\cdot, \cdot)$ and $b(\cdot, \cdot)$ are continuous bilinear forms defined by [4]:

$$
A(\Psi, \Phi)=A_{0}(\Psi, \Phi) \forall \Psi, \Phi \in \mathbf{V} \subset \mathbf{H} \text { such that }
$$

$$
|A(\Psi, \Phi)| \leq M\|\Psi\|_{\mathbf{V}}\|\Phi\|_{\mathbf{V}} \text { for some } M>0 ; \quad A(\Phi, \Phi) \geq \alpha\|\Phi\|_{\mathbf{H}}^{2} \forall \Phi \in \mathbf{V}, \text { for some } \alpha>0 \quad \text { [4] }
$$




$$
b(\Phi, v)=\int_{\Omega} \phi_{i j, j} v_{, i} \mathrm{~d} \Omega \forall \Phi \in \mathbf{V} \forall v \in W \text { with } b(\Phi, v)=b_{0}(\Phi, v) \forall \Phi \in \mathbf{V} \forall v \in H_{0}^{2}(\Omega)
$$

such that $|b(\Phi, v)| \leq m\|\Phi\|_{\mathbf{V}}\left\|_{v}\right\|_{W}, \forall \Phi \in \mathbf{V}, \forall v \in W$ and some $m>0 ;$ and $\exists \beta>0$ such that $\sup _{\Phi \in \mathbf{V}-\{\mathbf{0}\}} \frac{|b(\Phi, v)|}{\|\Phi\| \mathbf{v}} \geq \beta\|v\|_{W} \forall v \in W([4])$.

If $(\lambda ;(\Psi, u)) \in \mathbb{R} \times(\mathbf{V} \times W)$ be an eigenpair of $\left(\mathbf{Q}^{\mathbf{E}}\right)$, then $\lambda \in \mathbb{R}^{+}$.

Then, the corresponding continuous Mixed Variational Source Problem (Q) [4] is defined by: For given $f \in L^{2}(\Omega)$, find $(\Psi, u) \in \mathbf{V} \times W$ such that

$$
(\mathbf{Q}): A(\Psi, \Phi)+b(\Phi, u)=0 \quad \forall \Phi \in \mathbf{V}, \quad-b(\Psi, v)=\langle f, v\rangle_{0, \Omega} \forall v \in W .
$$

Since $A(\cdot, \cdot)$ is not $\mathbf{V}$-elliptic, $(\mathbf{Q})$ is not well-posed a priori. But we have:

Theorem 2.5 ( [4]). Let (A1-A5) hold. If $u \in H^{3}(\Omega) \cap H_{0}^{2}(\Omega)$ be the solution of the Galerkin Variational Source Problem $\left(\mathbf{P}_{\mathbf{G}}\right)$ with $\psi_{i j}=a_{i j k l} u_{, k l} \in H^{1}(\Omega) \forall i, j=1,2$ and $\Psi=\left(\psi_{i j}\right)_{1 \leq i, j \leq 2}$, then $(\Psi, u) \in \mathbf{V} \times W$ is the unique solution of $(\mathbf{Q})$. Conversely, let $(\Psi, u) \in \mathbf{V} \times W$ be the solution of $(\mathbf{Q})$. Then, $u \in H_{0}^{2}(\Omega)$ and is the unique solution of $\left(\mathbf{P}_{\mathbf{G}}\right)$ and $\psi_{i j}=a_{i j k l} u_{, k l} \quad \forall i, j=1,2 ; u_{, k l}=A_{i j k l} \psi_{i j} \quad \forall k, l=1,2 ; \quad \Psi=\left(\psi_{i j}\right)_{1 \leq i, j \leq 2}$.

Hence, under the assumption that the solution $u \in H_{0}^{2}(\Omega)$ of Galerkin Variational Source Problem $\left(\mathbf{P}_{\mathbf{G}}\right)$ in (2.4) has the additional regularity $[18,21,24]$ :

(A6) : $u \in H^{3}(\Omega) \cap H_{0}^{2}(\Omega)$ with $\|u\|_{3, \Omega} \leq C\|f\|_{0, \Omega}$ for some $C>0$,

the correspondence $f \in L^{2}(\Omega) \mapsto(\Psi, u) \in \mathbf{V} \times W$ with $u \in H^{3}(\Omega) \cap H_{0}^{2}(\Omega)$ defines an operator $\overrightarrow{\mathbf{T}} f=(S f, T f)=(\Psi, u) \in \mathbf{V} \times W$ with

$$
A(S f, \Phi)+b(\Phi, T f)=0 \quad \forall \Phi \in \mathbf{V}, \quad-b(S f, v)=\langle f, v\rangle_{0, \Omega} \forall v \in W
$$

$T: f \in L^{2}(\Omega) \mapsto T f=u \in H^{3}(\Omega) \cap H_{0}^{2}(\Omega), S: f \in L^{2}(\Omega) \mapsto S f=\Psi=\left(a_{i j k l}(T f)_{, k l}\right)_{i, j=1,2} \in \mathbf{V}$, being the solution component operators with $S(\cdot)=\left(a_{i j k l}(T(\cdot))_{, k l}\right)_{1 \leq i, j \leq 2}$ and $\|T f\|_{1, \Omega} \leq C\|f\|_{0, \Omega} ;\|S f\|_{1, \Omega} \leq C\|f\|_{0, \Omega}$; $\|S f\|_{0, \Omega}+\|T f\|_{1, \Omega} \leq C\|f\|_{0, \Omega}$.

Theorem 2.6 ( $[8,31])$. Under $(\mathbf{A 6})$, the source problems $(\mathbf{Q})$ and $\left(\mathbf{Q}_{\mathbf{A U X}}\right)$ are "equivalent" in the sense that these have the same solution $(\Psi, u) \in \mathbf{V} \times W$ with $u \in H^{3}(\Omega) \cap H_{0}^{2}(\Omega)$, $\psi_{i j}=a_{i j k l} u_{, k l} \in H^{1}(\Omega) \forall i, j=1,2, \Psi=\left(\psi_{i j}\right)_{1 \leq i, j \leq 2} \in \mathbf{V} \subset \mathbf{H}$.

Hence under (A6), $\forall f \in L^{2}(\Omega), S f=S_{0} f=\Psi \in \mathbf{V} \subset \mathbf{H}, T f=T_{0} f=u \in H^{3}(\Omega) \cap H_{0}^{2}(\Omega) \subset W \subset L^{2}(\Omega)$ and all the results associated with $T_{0} \in \mathcal{L}\left(L^{2}(\Omega)\right)$ and $S_{0} \in \mathcal{L}\left(L^{2}(\Omega)\right)$ will hold for $T \in \mathcal{L}\left(L^{2}(\Omega)\right)$ and $S \in$ $\mathcal{L}\left(L^{2}(\Omega) ; \mathbf{V}\right)$. Hence, we have the important result:

Theorem 2.7 ( [8]). Under (A6), mixed variational eigenvalue problems $\left(\mathbf{Q}^{\mathbf{E}}\right)$ and $\left(\mathbf{Q}_{\mathbf{A}}^{\mathbf{E}} \mathbf{X}\right)$ are equivalent in the sense that both of these eigenvalue problems have the same strictly positive eigenvalues $\left(\lambda_{m}\right)_{m=1}^{\infty}$ and the same eigenpairs $\left(\lambda_{m} ;\left(\Psi_{m}, u_{m}\right)\right) \in \mathbb{R}^{+} \times(\mathbf{V} \times W)$ with $u_{m} \in H^{3}(\Omega) \cap H_{0}^{2}(\Omega), \quad\left(u_{m}\right)_{m=1}^{\infty}$ being a Hilbert basis in $L^{2}(\Omega)$ and $\left(\Psi_{m} / \sqrt{\lambda_{m}}\right)_{m=1}^{\infty}$ being an orthonormal system in $(\mathbf{H}, A(\cdot, \cdot))$.

Define a linked pair $\left(\underline{\sigma}_{p}, \chi_{p}\right)=\sum_{m=1}^{p} c_{m}\left(\Psi_{m}, u_{m}\right) \in \mathcal{M}_{p}$ with $\underline{\sigma}_{p}=\sum_{m=1}^{p} c_{m} \Psi_{m} \in \mathbf{V}$, $\chi_{p}=\sum_{m=1}^{p} c_{m} u_{m} \in H^{3}(\Omega) \cap H_{0}^{2}(\Omega) c_{m} \in \mathbb{R} \forall m=1,2, \cdots, p$, where $\left(\Psi_{m}, u_{m}\right) \in \mathbf{V} \times W$ with $u_{m} \in H^{3}(\Omega) \cap H_{0}^{2}(\Omega)$ is an eigenelement of $\left(\mathbf{Q}^{\mathbf{E}}\right)$ corresponding to the eigenvalue $\lambda_{m}, 0<\lambda_{1} \leq \lambda_{2} \leq \cdots \lambda_{p}$. In 
particular, for $c_{m}=0 \forall m \neq p, c_{p}=1, \underline{\sigma}_{p}=\Psi_{p}, \chi_{p}=u_{p}$. Then, $\forall\left(\underline{\sigma}_{p}, \chi_{p}\right) \in \mathcal{M}_{p}$ with $\chi_{p} \in H^{3}(\Omega) \cap H_{0}^{2}(\Omega)$ and $\underline{\sigma}_{p} \in \mathbf{V}, \exists$ a unique linked pair $\left(\underline{\sigma}_{p}^{*}, \chi_{p}^{*}\right) \in \mathcal{M}_{p}$ with

$$
\chi_{p}^{*}=\sum_{m=1}^{p} \lambda_{m} c_{m} u_{m} \text { and } \underline{\sigma}_{p}^{*}=\mathcal{I} \chi_{p}^{*}=\sum_{m=1}^{p} \lambda_{m} c_{m} \Psi_{m}
$$

such that $\overrightarrow{\mathbf{T}} \chi_{p}^{*}=\left(\underline{\sigma}_{p}, \chi_{p}\right), \overrightarrow{\mathbf{T}}$ being the linear operator defined in $(2.25)$.

Examples [8]: I. Biharmonic Eigenvalue Problem is obtained from $(2.2) a_{i j k l}$ defined by: $a_{i i i i}=$ 1; $a_{1212}=a_{2121}=a_{2112}=a_{1221}=1 / 2 ; a_{i j k l}=0$ otherwise, which satisfy (A3-A4) [3], in $\widetilde{\Omega}$. Then, we have $\Lambda \equiv \Delta \Delta$, for which (A6) holds [4]. $\left(\mathbf{Q}^{\mathbf{E}}\right)$ corresponds to $\mathbf{H}-\mathbf{H}-\mathbf{M}$ (Hellan-Hermann-Miyoshi) mixed method scheme for biharmonic eigenvalue problem [4,13,30]. $\forall m \in \mathbb{N}\left(\lambda_{m} ;\left(\Psi_{m}, u_{m}\right)\right) \in \mathbb{R}^{+} \times(\mathbf{V} \times W)$ with $u_{m} \in H^{3}(\Omega) \cap H_{0}^{2}(\Omega)$ and $\Psi_{m}=\left(\psi_{m i j}\right)_{i, j=1,2}$ is an eigenpair of biharmonic eigenvalue problem in $\mathbf{H}-\mathbf{H}-\mathbf{M}$ mixed method formulation $[2,13,14,22]$ :

$$
\int_{\Omega} \psi_{m i j} \phi_{i j} \mathrm{~d} \Omega+\int_{\Omega} \phi_{i j, j} u_{m, i} \mathrm{~d} x=0 \forall \Phi \in \mathbf{V}, \quad-\int_{\Omega} \psi_{m i j, j} v_{, i} \mathrm{~d} \Omega=\lambda_{m}\left\langle u_{m}, v\right\rangle_{0, \Omega} \forall v \in W .
$$

Remark 2.1. The associated biharmonic source problem corresponds to Stokes problem [34] of fluid mechanics in stream function-vorticity formulation and also to the bending problem of isotropic elastic plates with flexural rigidity $D=1, \nu=0$ (see $(2.32)$ ).

II. Eigenvalue problems associated with the vibration of elastic plates with variable/ constant thickness. (i) In Anisotropic case [4,27],

$$
\begin{aligned}
& a_{i i i i}=D_{i i}, a_{1212}=a_{1221}=a_{2121}=a_{2112}=D_{66}, a_{1112}=a_{1211}=a_{2111}=a_{1121}=D_{16}, \\
& a_{1222}=a_{2122}=a_{2212}=a_{2221}=D_{26}, a_{2211}=a_{1122}=D_{12},
\end{aligned}
$$

$D_{i j}=D_{i j}\left(x_{1}, x_{2}\right) \quad \forall\left(x_{1}, x_{2}\right) \in \overline{\widetilde{\Omega}}$ being rigidities [8,27] for which (A2-A4) hold, and the anisotropic plate bending operator $\Lambda$ is given by:

$$
\begin{aligned}
\Lambda u \equiv & \left(D_{11} u_{, 11}+2 D_{16} u_{, 12}+D_{12} u_{, 22}\right)_{, 11}+2\left(D_{16} u_{, 11}+2 D_{66} u_{, 12}+D_{26} u_{, 22}\right)_{, 12} \\
& +\left(D_{12} u_{, 11}+2 D_{26} u_{, 12}+D_{22} u_{, 22}\right)_{, 22} .
\end{aligned}
$$

Then, coefficients $A_{i j k l}$ are defined in terms of $D_{i j}$ 's $[4,9,10]$ and the corresponding bilinear form $A(\cdot, \cdot)$ of $\left(\mathbf{Q}^{\mathbf{E}}\right)$ is given by: $\Psi, \Phi \in \mathbf{V}$,

$$
\begin{aligned}
A(\Psi, \Phi)= & \int_{\Omega} \frac{4}{|A(x)|}\left[\left\{\left(D_{22} D_{66}-D_{26}^{2}\right) \psi_{11}+\left(D_{16} D_{26}-D_{12} D_{66}\right) \psi_{22}+\left(D_{12} D_{26}-D_{16} D_{22}\right) \psi_{12}\right\} \phi_{11}\right. \\
& +\left\{\left(D_{16} D_{26}-D_{12} D_{66}\right) \psi_{11}+\left(D_{11} D_{66}-D_{16}^{2}\right) \psi_{22}+\left(D_{16} D_{12}-D_{11} D_{26}\right) \psi_{12}\right\} \phi_{22} \\
& \left.+\left\{\left(D_{12} D_{26}-D_{16} D_{22}\right) \psi_{11}+\left(D_{16} D_{12}-D_{11} D_{26}\right) \psi_{22}+\left(D_{11} D_{22}-D_{12}^{2}\right) \psi_{12}\right\} \phi_{12}\right] \mathrm{d} x
\end{aligned}
$$

where $|A(x)|=4\left(D_{11} D_{22} D_{66}-D_{11} D_{26}^{2}-D_{66} D_{12}^{2}-D_{22} D_{16}^{2}+D_{12} D_{16} D_{26}\right)(x)$.

(ii) The Orthotropic case $[3,27,38]$ can be retrieved from the anisotropic case (i) by putting in $(2.28)-(2.30)$,

$$
\begin{aligned}
& a_{i i i i}=D_{i} ; a_{1122}=a_{2211}=D_{12}=\nu_{1} D_{2}=\nu_{2} D_{1} \\
& a_{1212}=a_{2121}=a_{2112}=a_{1221}=D_{\tau}, a_{i j k l}=0 \text { otherwise, }
\end{aligned}
$$


with $D_{i}=D_{i}\left(x_{1}, x_{2}\right)$ and $D_{\tau}=D_{\tau}\left(x_{1}, x_{2}\right) \forall\left(x_{1}, x_{2}\right) \in \overline{\widetilde{\Omega}}, H=D_{1} \nu_{2}+2 D_{\tau}, \nu_{i}, i=1,2$ being Poisson's coefficients respectively, $D_{i}$ 's and $D_{\tau}$ being rigidities, assumptions (A3-A4) hold [3] and

(iii) the Isotropic case is obtained from the Orthotropic case by putting $\nu_{1}=\nu_{2}=\nu$ and $D_{1}=D_{2}=D$ in all formulae (2.31). Then, the orthotropic (resp. isotropic) plate (bending) operator $\Lambda$ and the corresponding bilinear form $A(\cdot, \cdot)$ of $\left(\mathbf{Q}^{\mathbf{E}}\right)$ are given by:

Orthotropic Case: $\Lambda u \equiv\left(D_{1} u_{, 11}+\nu_{2} D_{1} u_{, 22}\right)_{, 11}+4\left(D_{\tau} u_{, 12}\right)_{, 12}+\left(\nu_{1} D_{2} u_{, 11}+D_{2} u_{, 22}\right)_{, 22}$ $A(\Psi, \Phi)=\int_{\Omega}\left[\frac{1}{D_{1}\left(1-\nu_{1} \nu_{2}\right)}\left(\psi_{11}-\nu_{1} \psi_{22}\right) \phi_{11}+\frac{1}{D_{2}\left(1-\nu_{1} \nu_{2}\right)}\left(-\nu_{2} \psi_{11}+\psi_{22}\right) \phi_{22}+\frac{1}{D_{\tau}} \psi_{12} \phi_{12}\right] \mathrm{d} x \quad \forall \Psi, \Phi \in \mathbf{V}$. Isotropic Case: $\Lambda u \equiv\left(D\left(u_{, 11}+\nu u_{, 22}\right)\right)_{, 11}+2\left(D(1-\nu) u_{, 12}\right)_{, 12}+\left(D\left(\nu u_{, 11}+u_{, 22}\right)\right)_{, 22}$.

Then, for $D=$ constant $, \Lambda u \equiv D \Delta \Delta u,(\mathbf{A 6})$ will hold $[18,21,24]$.

$A(\Psi, \Phi)=\int_{\Omega}\left[\frac{1}{D\left(1-\nu^{2}\right)}\left(\psi_{11}-\nu \psi_{22}\right) \phi_{11}+\frac{1}{D\left(1-\nu^{2}\right)}\left(-\nu \psi_{11}+\psi_{22}\right) \phi_{22}+\frac{2}{D(1-\nu)} \psi_{12} \phi_{12}\right] \mathrm{d} x \forall \Psi, \Phi \in \mathbf{V}$. In aniso-/ortho-/isotropic cases (i-iii), $\forall$ eigenpair $\left(\lambda_{m} ;\left(\Psi_{m}, u_{m}\right)\right)$ of $\left(\mathbf{Q}^{\mathbf{E}}\right), u_{m}$ is the deflection mode of the vibrating plate, $\Psi_{m}=\left(\psi_{m i j}\right)_{1 \leq i, j \leq 2}$ is the corresponding bending moment tensor, $\psi_{m i i}$ being the bending moment in the $x_{i}$ direction and $\psi_{m 12}=\psi_{m 21}$, being the twisting moment, i.e.

Anisotropic Case: $\psi_{m i j}=a_{i j k l} u_{, k l}$ with $a_{i j k l}$ 's defined by $(2.28), i, j=1,2$;

Orthotropic Case: $\psi_{m 11}=D_{1}\left(u_{m, 11}+\nu_{2} u_{m, 22}\right) ; \psi_{m 22}=D_{2}\left(\nu_{1} u_{m, 11}+u_{m, 22}\right), \psi_{m 12}=2 D_{\tau} u_{m, 12}$;

Isotropic Case: $\psi_{m 11}=D\left(u_{m, 11}+\nu u_{m, 22}\right), \psi_{m 22}=D\left(\nu u_{m, 11}+u_{m, 22}\right), \psi_{m 12}=\psi_{m 21}=D(1-\nu) u_{m, 12}$.

Remark 2.2. In the orthotropic plates with constant thickness, $D_{1}=$ constant, $D_{2}=$ constant, $H=D_{1} \nu_{2}+$ $2 D_{\tau}=$ constant and $\Lambda u \equiv D_{1} u_{, 1111}+2 H u_{, 1122}+D_{2} u_{, 2222}$.

$$
\text { Then, for } H=\sqrt{D_{1} D_{2}}, \Lambda u \equiv D_{1} u, 1111+2 \sqrt{D_{1} D_{2}} u_{, 1122}+D_{2} u_{, 2222}
$$

can be reduced to the form (2.32) by introducing a new variable $\xi_{2}=x_{2}\left(D_{1} / D_{2}\right)^{1 / 4}, \xi_{1}=x_{1}$ ( [38], pp. 366367), i.e. $\Lambda u^{*} \equiv D_{1} \Delta \Delta u^{*}$ with $u^{*}=u^{*}\left(\xi_{1}, \xi_{2}\right), \Delta=\frac{\partial^{2}}{\partial \xi_{1}^{2}}+\frac{\partial^{2}}{\partial \xi_{2}^{2}}$. Hence, (A6) will also hold for (2.33) [18,21,24].

\section{Isoparametric mixed finite Element eigenvalue PRoblem $\left(\mathbf{Q}_{\mathbf{h}}^{\mathbf{E}}\right)$}

Isoparametric Triangulation $\tau_{h}^{\text {ISO }}$ : Let $\left\{P_{i}\right\}_{i=1}^{N_{c}}$ be $N_{c}$ corner points of $\Gamma$ at which $C^{m}$-smoothness $(m \geq 3)$ does not hold and $\left\{P_{j}\right\}_{N_{c}}^{N_{h}}$ be the set of possible additional points suitably chosen on $\Gamma$ such that $\nu_{h}=$ $\left\{P_{i}\right\}_{i=1}^{N_{c}} \cup\left\{P_{j}\right\}_{j=N_{c}+1}^{N_{h}} \subset \Gamma$ denote the set of boundary vertices of the isoparametric triangulation of $\bar{\Omega}$ under consideration.

I. $\tau_{h}^{\text {pol }}$ : Let $\tau_{h}^{\text {pol }}=\tilde{\tau}_{h}^{b} \cup \tau_{h}^{0}$ be the admissible, regular, quasi-uniform triangulation of the closed polygonal domain $\bar{\Omega}_{h}^{\text {pol }}=\Omega_{h}^{\text {pol }} \cup \Gamma_{h}^{\text {pol }}$ with vertices $\left\{P_{i}\right\}_{i=1}^{N_{h}}$ into closed triangles $\tilde{T}$ with vertices $\left(a_{i, \tilde{T}}\right)_{i=1}^{3}$ and the straight sides $\left(\partial \tilde{T}_{i}\right)_{i=1}^{3}, \quad \partial \tilde{T}_{i}=\left[a_{i, \tilde{T}}, a_{i+1, \tilde{T}}\right]$ (modulo 3) such that $\Gamma_{h}^{\text {pol }}=\cup_{\tilde{T} \in \tilde{T}_{h}^{b}} \partial \tilde{T}_{1}, \quad \partial \tilde{T}_{1}=\left[a_{1, \tilde{T}}, a_{2, \tilde{T}}\right]$ being the boundary side $\forall \tilde{T} \in \tilde{\tau}_{h}^{b}$,

$$
\tilde{\tau}_{h}^{b}=\left\{\tilde{T}: \tilde{T} \in \tau_{h}^{\text {pol }} \text { is a boundary triangle with single boundary side } \partial \tilde{T}_{1}\right\} ;
$$

$\tau_{h}^{0}=\left\{\tilde{T}: \tilde{T} \in \tau_{h}^{\mathrm{pol}}\right.$ is an interior triangle with at most one of its vertices lying on $\left.\Gamma\right\} ;$

II. $\tau_{h}^{\text {exact }}$ : Keeping all vertices of $\tau_{h}^{\text {pol }}$ and interior triangles $\tilde{T} \in \tau_{h}^{0}$ in (3.2) undisturbed and replacing each boundary triangle $\tilde{T} \in \tilde{\tau}_{h}^{b}$ by a curved boundary triangle $\bar{T}$ which is obtained by replacing the straight 
boundary side $\partial \tilde{T}_{1}$ of $\tilde{T} \in \tilde{\tau}_{h}^{b}$ by a part $\partial \bar{T}_{1}$ of the boundary $\Gamma$ between the boundary vertices of $\tilde{T} \in \tilde{\tau}_{h}^{b}$. Let $\bar{\tau}_{h}^{b}$ denote all such curved boundary triangles $\bar{T}$. Then, $\tau_{h}^{\text {exact }}=\bar{\tau}_{h}^{b} \cup \tau_{h}^{0}$ with $\bar{\Omega}=\cup_{\bar{T} \in \tau_{h}^{\text {exact }}} \bar{T}$ and $\Gamma=\cup_{\bar{T} \in \tilde{\tau}_{h}^{b}} \partial \bar{T}_{1}$. III. $\tau_{h}^{\text {ISO }}$ : Again, we keep all vertices of $\tau_{h}^{\text {pol }}$ and interior triangles $\tilde{T} \in \tau_{h}^{0}$ undisturbed. $\forall$ boundary triangle $\tilde{T} \in \tilde{\tau}_{h}^{b}$ in (3.1), define mid-side points $a_{4, \tilde{T}}=\left(a_{1, \tilde{T}}+a_{2, \tilde{T}}\right) / 2, a_{5, \tilde{T}}=\left(a_{2, \tilde{T}}+a_{3, \tilde{T}}\right) / 2, a_{6, \tilde{T}}=\left(a_{3, \tilde{T}}+a_{1, \tilde{T}}\right) / 2$. To $a_{4, \tilde{T}} \in \Gamma_{h}^{\text {pol }}$, we associate the point $a_{4, T}^{*} \in \Gamma$ as the point of intersection of the perpendicular bisector of $\partial \tilde{T}_{1}$ at $a_{4, \tilde{T}}$ with $\Gamma$. Let $\hat{T}$ be the reference triangle with vertices $\hat{a}_{1}=(1,0), \hat{a}_{2}=(0,1), \hat{a}_{3}=(0,0)$ and mid-side nodes $\hat{a}_{4}=(1 / 2,1 / 2), \hat{a}_{5}=(0,1 / 2), \hat{a}_{6}=(1 / 2,0)$, sides $\partial \hat{T}_{i}=\left[\hat{a}_{i}, \hat{a}_{i+1}\right]$ (modulo 3$) \quad 1 \leq i \leq 3$. Then, with the help of canonical basis functions $\left(\hat{\phi}_{i}\right)_{i=1}^{6}$ of $P_{2}(\hat{T})\left(i . e . \forall \hat{\phi}_{i} \in P_{2}(\hat{T}), \hat{\phi}_{i}\left(\hat{a}_{j}\right)=\delta_{i j}, \quad 1 \leq i, j \leq 2\right)$, define the invertible isoparametric mapping by: $\forall \hat{x} \in \hat{T}$,

$$
F_{T}(\hat{x})=\sum_{i=1}^{3} a_{i, \tilde{T}} \hat{\phi}_{i}(\hat{x})+\sum_{i=5}^{6} a_{i, \tilde{T}} \hat{\phi}_{i}(\hat{x})+a_{4, T}^{*} \hat{\phi}_{4}(\hat{x})=x \in T=F_{T}(\hat{T}),
$$

such that $F_{T}\left(\hat{a}_{i}\right)=a_{i, \tilde{T}} \in \tilde{T} \in \tilde{\tau}_{h}^{b}, 1 \leq i \neq 4 \leq 6, F_{T}\left(\hat{a}_{4}\right)=a_{4, T}^{*} \in \Gamma, \quad F_{T}\left(\partial \hat{T}_{i}\right)=\partial T_{i}, \quad 1 \leq i \leq 3$. Then, $\forall \tilde{T} \in \tilde{\tau}_{h}^{b}$ in (3.1), we get a curved boundary triangle $T=F_{T}(\hat{T})$ with the single curved boundary side $\partial T_{1}=F_{T}\left(\partial \hat{T}_{1}\right)$. Let $\tau_{h}^{b}$ be all such curved boundary triangles. Then,

$$
\tau_{h}^{\mathrm{ISO}}=\tau_{h}^{b} \cup \tau_{h}^{0} \text { with } \tau_{h}^{0} \text { defined by }(3.2), \quad \bar{\Omega}_{h}=\cup_{T \in \tau_{h}^{\mathrm{ISO}}} T, \Gamma_{h}=\cup_{T \in \tau_{h}^{b}} \partial T_{1}
$$

is the Isoparametric Triangulation of $\bar{\Omega}, \Gamma_{h}$ being the approximation of the boundary $\Gamma$. For other methods of approximation of boundary $\Gamma$, we refer to [7,41]. Such a $\tau_{h}^{\text {ISO }}$ is regular in the sense of [16]. $\Omega_{h}$ is not convex, $\Omega_{h} \not \subset \Omega, \Omega \not \subset \Omega_{h}$ in general. But by construction, the distance of $\Gamma$ from $\Gamma_{h}$ tends to 0 as $h \longrightarrow 0$ and from $(\mathbf{A 1}), \quad \exists \widetilde{\Omega}$ with boundary $\widetilde{\Gamma}$, which is piecewise of $C^{k}$-class, $k \geq 3$, such that $\bar{\Omega} \subset \widetilde{\Omega}$. Hence,

(A7): $\exists h_{0}>0$ such that $\left.\forall h \in\right] 0, h_{0}\left[, \bar{\Omega}_{h} \subset \widetilde{\Omega}\right.$.

Then, from $(\mathbf{A 1})$ and $(\mathbf{A 7}) \forall h \in] 0, h_{0}\left[, \bar{\Omega}_{h} \subset \widetilde{\Omega}, \bar{\Omega} \subset \widetilde{\Omega}\right.$ with $\left(\bar{\Omega}_{h} \cup \bar{\Omega}\right) \subset \widetilde{\Omega}$ and define

$$
\begin{aligned}
& \epsilon_{h}=\cup_{T \in \tau_{h}^{b}, \bar{T} \in \bar{\tau}_{h}^{b}}\left(T^{\mathrm{int}}-\left(\bar{T}^{\mathrm{int}} \cap T^{\mathrm{int}}\right)\right)=\Omega_{h}-\left(\Omega \cap \Omega_{h}\right) \text { with meas }\left(\epsilon_{h}\right)=O\left(h^{3}\right) \quad[10,17] ; \\
& \omega_{h}=\cup_{\bar{T} \in \bar{\tau}_{h}^{b}, T \in \tau_{h}^{b}}\left(\bar{T}^{\mathrm{int}}-\left(\bar{T}^{\mathrm{int}} \cap T^{\mathrm{int}}\right)\right)=\Omega-\left(\Omega \cap \Omega_{h}\right) \text { with meas }\left(\omega_{h}\right)=O\left(h^{3}\right) \quad[10,17],
\end{aligned}
$$

where $\left.T^{\text {int }}=\operatorname{int}(T), \bar{T}^{\text {int }}=\operatorname{int}(\bar{T}), \tau_{h}^{b} \subset \tau_{h}^{\text {ISO }}, \bar{\tau}_{h}^{b} \subset \tau_{h}^{\text {exact }} \forall h \in\right] 0, h_{0}\left[\right.$ with $h_{0}>0$.

$\forall h$ boundary $\Gamma_{h}$ of $\Omega_{h}$ is piecewise of $C^{\infty}$-class, $\nu_{h}$ being the set of boundary vertices of $\tau_{h}^{\text {ISO }}$ at which $C^{\infty}$ smoothness does not hold. For the properties of the invertible $F_{T}\left(\right.$ resp. $\left.F_{T}^{-1}\right)$ and its Jacobian $J\left(F_{T}\right) \in P_{1}(\hat{T})$ with important estimates, we refer to $[10,16,17]$.

We will need extensions to $\mathbb{R}^{2}$ of functions defined in $\Omega_{h}$ (resp. $\Omega$ ).

Theorem 3.1 ( $[32,39])$. Let $\mathcal{D}$ be a bounded, two-dimensional domain with Lipschitz continuous boundary $\partial \mathcal{D}$, which is piecewise of $C^{k}$-class, $k \geq 1$. Then,

(a) $\exists$ a continuous, linear extension operator $E: H^{k}(\mathcal{D}) \longrightarrow H^{k}\left(\mathbb{R}^{2}\right)$, i.e. $\exists C>0$ such that

$$
\|E u\|_{k, \mathbb{R}^{2}}=\|\tilde{u}\|_{k, \mathbb{R}^{2}} \leq C\|u\|_{k, \mathcal{D}} \forall \text { fixed } k \geq 1 \text { with } E u \downarrow_{\mathcal{D}}=\tilde{u} \downarrow_{\mathcal{D}}=u \in H^{k}(\mathcal{D}) \text {. }
$$

(b) The operator $E$ is also a linear and bounded extension operator from $H^{(k-i)}(\mathcal{D})$ into $H^{(k-i)}\left(\mathbb{R}^{2}\right), 1 \leq i \leq k$, i.e. $\exists C>0$ such that $\|E u\|_{k-i, \mathbb{R}^{2}}=\|\tilde{u}\|_{k-i, \mathbb{R}^{2}} \leq C\|u\|_{k-i, \mathcal{D}}, 1 \leq i \leq k$, with $E u \downarrow_{\mathcal{D}}=\tilde{u} \downarrow_{\mathcal{D}}=u \in H^{k-i}(\overline{\mathcal{D}}), 1 \leq$ $i \leq k$, and in particular,

$$
\|\tilde{u}\|_{0, \mathbb{R}^{2}} \leq C\|u\|_{0, \mathcal{D}} \quad \forall u \in L^{2}(\mathcal{D}) \text { with } \tilde{u} \in L^{2}\left(\mathbb{R}^{2}\right)
$$


Boundaries $\Gamma_{h}, \Gamma, \tilde{\Gamma}$ of $\Omega_{h}, \Omega$ and $\widetilde{\Omega}$ respectively satisfy the smoothness conditions in Theorem 3.1. We can choose $\mathcal{D}=\Omega_{h}$ (resp. $\Omega$ ) and find the corresponding extension $\tilde{u}_{h}=E u_{h}($ resp. $\tilde{u}=E u)$.

Corollary 3.1. For $\mathcal{D}=\Omega_{h}($ resp. $\Omega)$, let $E: H^{k}(\mathcal{D}) \longrightarrow H^{k}\left(\mathbb{R}^{2}\right)$, be the continuous linear extension operator defined in (3.7) and (3.8) and $\rho: H^{k}\left(\mathbb{R}^{2}\right) \longrightarrow H^{k}(\widetilde{\Omega})$ be the continuous, linear restriction operator i.e. $\forall \tilde{u} \in$ $H^{k}\left(\mathbb{R}^{2}\right), \quad\|\rho \tilde{u}\|_{k, \widetilde{\Omega}}=\|\tilde{u}\|_{k, \widetilde{\Omega}} \leq C\|\tilde{u}\|_{k, \mathbb{R}^{2}}$ with $\tilde{u} \downarrow_{\widetilde{\Omega}}=\tilde{u}$. (For restriction to $\widetilde{\Omega}$ of $\tilde{u}$, the same notation $\tilde{u}$ has been used and will be used in the sequel).

Then, $\rho \cdot E: H^{k}(\mathcal{D}) \longrightarrow H^{k}(\widetilde{\Omega})$ is a continuous, linear extension operator from $H^{k}(\mathcal{D})$ into $H^{k}(\widetilde{\Omega})$, i.e. $\forall u \in$ $H^{k}(\mathcal{D}),\|\rho \cdot E u\|_{k, \widetilde{\Omega}}=\|\tilde{u}\|_{k, \widetilde{\Omega}} \leq C\|u\|_{k, \mathcal{D}}$ for some $C>0$

$$
\text { and }\|\tilde{u}\|_{k-i, \widetilde{\Omega}} \leq C\|u\|_{k-i, \mathcal{D}} \quad \forall i=1,2, \cdots, k \text {. }
$$

(In (3.7) and (3.9), the same letter $C>0$, having different strictly positive values has been used and this convention of using the same letter $C>0$ with different strictly positive values at different steps will be followed in the sequel unless stated otherwise).

With the help of Theorem 3.1 and Corollary 3.1, we define $\widetilde{\mathbf{V}}$ and $\widetilde{W}$ as follows:

$$
\begin{aligned}
\widetilde{\mathbf{V}}=\left\{\widetilde{\Phi}: \widetilde{\Phi}=\left(\widetilde{\phi}_{i j}\right)_{i, j=1,2} ; \widetilde{\phi}_{i j}=\widetilde{\phi}_{j i} \in H^{1}(\widetilde{\Omega}) \text { such that } \widetilde{\phi}_{i j} \downarrow_{\Omega}=\phi_{i j} \in H^{1}(\Omega),\right. \\
\\
\left.\Phi=\left(\phi_{i j}\right)_{i, j=1,2} \in \mathbf{V}\right\} \text { with }\left\|\widetilde{\phi}_{i j}\right\|_{1, \widetilde{\Omega}} \leq C\left\|\phi_{i j}\right\|_{1, \Omega} \forall i, j=1,2, \text { for some } C>0 ; \\
\widetilde{W}=\left\{\widetilde{\chi}: \widetilde{\chi} \in H_{0}^{1}(\widetilde{\Omega}), \widetilde{\chi} \downarrow_{\widetilde{\Omega}-\Omega}=0\right\} \text { with }\|\widetilde{\chi}\|_{\widetilde{W}}=\|\widetilde{\chi}\|_{H_{0}^{1}(\widetilde{\Omega})}=\|\chi\|_{1, \Omega} .
\end{aligned}
$$

To every $\bar{\Omega}_{h}$ we associate Hilbert spaces $\mathbf{V}\left(\Omega_{h}\right)$ and $H_{0}^{1}\left(\Omega_{h}\right)$ defined by:

$\mathbf{V}\left(\Omega_{h}\right)=\left\{\Phi: \Phi=\left(\phi_{i j}\right)_{i, j=1,2}, \phi_{i j}=\phi_{j i} \in H^{1}\left(\Omega_{h}\right) \quad \forall i, j=1,2\right\}$ with $\|\Phi\|_{\mathbf{V}\left(\Omega_{h}\right)}^{2}=\|\Phi\|_{1, \Omega_{h}}^{2}=\sum_{i=1}^{2} \sum_{j=1}^{2}\left\|\phi_{i j}\right\|_{1, \Omega_{h}}^{2}$, $H_{0}^{1}\left(\Omega_{h}\right)=\left\{v: v \in H^{1}\left(\Omega_{h}\right),\left.v\right|_{\Gamma_{h}}=0\right\}$ with $\|v\|_{H_{0}^{1}\left(\Omega_{h}\right)}=\|v\|_{1, \Omega_{h}}$,

and define the auxiliary continuous, bilinear forms $\widetilde{A}_{h}(\cdot, \cdot)$ and $\widetilde{b}_{h}(\cdot, \cdot)$ by:

$$
\begin{gathered}
\widetilde{A}_{h}(\Psi, \Phi)=\int_{\Omega_{h}} A_{i j k l} \psi_{i j} \phi_{k l} \mathrm{~d} x=\widetilde{A}_{h}(\Phi, \Psi) \forall \Psi, \Phi \in \mathbf{V}\left(\Omega_{h}\right), \\
\widetilde{b}_{h}(\Phi, v)=\int_{\Omega_{h}} \phi_{i j, j} v, i \mathrm{~d} x \quad \forall \Phi \in \mathbf{V}\left(\Omega_{h}\right) \forall v \in H_{0}^{1}\left(\Omega_{h}\right)
\end{gathered}
$$

with $\left|\tilde{A}_{h}(\Phi, \Psi)\right| \leq \tilde{M}\|\Phi\|_{0, \Omega_{h}}\|\Psi\|_{0, \Omega_{h}}$ and $\left|\tilde{b}_{h}(\Phi, v)\right| \leq \tilde{m}\|\Phi\|_{1, \Omega_{h}}\|v\|_{1, \Omega_{h}}$ for some $\tilde{M}, \tilde{m}>0$.

Quadrature schemes: For $\hat{\phi} \in C^{0}(\hat{T})$, the following two quadrature schemes over the reference triangle $\hat{T}$ corresponding to $i=0$ and 1 will be considered:

$$
\int_{\hat{T}} \hat{\phi}(\hat{x}) \mathrm{d} \hat{x} \approx \sum_{n=1}^{N_{i}} \hat{w}_{n}^{i} \hat{\phi}\left(\hat{b}_{n}^{i}\right) \text { with weights } \hat{w}_{n}^{i}>0, \hat{b}_{n}^{i} \in \hat{T}, 1 \leq n \leq N_{i}, i=0,1 \text { such that }
$$


$\hat{E}_{0}(\hat{p})=\int_{\hat{T}} \hat{p}(\hat{x}) \mathrm{d} \hat{x}-\sum_{n=1}^{N_{0}} \hat{w}_{n}^{0} \hat{p}\left(\hat{b}_{n}^{0}\right)=0 \quad \forall \hat{p} \in P_{3}(\hat{T}) ; \hat{E}_{1}(\hat{p})=\int_{\hat{T}} \hat{p}(\hat{x}) \mathrm{d} \hat{x}-\sum_{n=1}^{N_{1}} \hat{w}_{n}^{1} \hat{p}\left(\hat{b}_{n}^{1}\right)=0 \quad \forall \hat{p} \in P_{6}(\hat{T})$.

Then, $\forall T \in \tau_{h}^{\mathrm{ISO}}, J\left(F_{T}\right)>0, J\left(F_{T}\right) \in P_{1}(\hat{T}), \forall \phi \in C^{0}(T)$,

$$
\int_{T} \phi(x) \mathrm{d} x=\int_{\hat{T}} \hat{\phi}(\hat{x}) J\left(F_{T}\right)(\hat{x}) \mathrm{d} \hat{x} \approx \sum_{n=1}^{N_{i}} w_{n, T}^{i} \phi\left(b_{n, T}^{i}\right) \text { with } \phi(x)=\phi \cdot F_{T}(\hat{x})=\hat{\phi}(\hat{x})
$$

where $w_{n, T}^{i}=\hat{w}_{n}^{i} J\left(F_{T}\right)\left(\hat{b}_{n}^{i}\right)>0, \quad b_{n, T}^{i}=F_{T}\left(\hat{b}_{n}^{i}\right) \in T$ satisfying the assumption:

(A8): Evaluation points $\hat{b}_{n}^{i} \in \hat{T}$ are vertices $\left\{\hat{a}_{i}\right\}_{i=1}^{3}$, midside nodes $\left\{\hat{a}_{i}\right\}_{i=4}^{6}$ and possibly also interior points $\hat{b}_{n}^{i} \in \operatorname{int}(\hat{T})$ such that $F_{T}\left(\hat{b}_{n}^{i}\right)=b_{n, T}^{i} \in T \cap \bar{\Omega} \forall T \in \tau_{h}^{\mathrm{ISO}}, 1 \leq n \leq N_{i}, \quad(i=0,1)$.

Then, to each $\tau_{h}^{\text {ISO }}$ of $\bar{\Omega}$, we associate the following finite dimensional subspaces:

$$
\begin{gathered}
X_{h}=\left\{\phi_{h}: \phi_{h} \in C^{0}\left(\bar{\Omega}_{h}\right), \phi_{h} \downarrow_{T}=\hat{\phi} \cdot F_{T}^{-1} \text { with } \hat{\phi} \in P_{2}(\hat{T}) \quad \forall T \in \tau_{h}^{\mathrm{ISO}}\right\} \subset H^{1}\left(\Omega_{h}\right) ; \\
\mathbf{V}_{h}=\left\{\Phi_{h}: \Phi_{h}=\left(\phi_{h i j}\right)_{i, j=1,2} \text { with } \phi_{h i j}=\phi_{h j i} \in X_{h} \forall i, j=1,2\right\} \subset \mathbf{V}\left(\Omega_{h}\right) ; \\
W_{h}=\left\{\chi_{h}: \chi_{h} \in X_{h},\left.\chi_{h}\right|_{\Gamma_{h}}=0\right\} \subset H_{0}^{1}\left(\Omega_{h}\right) \text { with }\left\|\chi_{h}\right\|_{W_{h}}=\left\|\chi_{h}\right\|_{1, \Omega_{h}},
\end{gathered}
$$

(in which the essential boundary condition $\left.\chi\right|_{\Gamma}=0$ has been replaced by $\left.\chi_{h}\right|_{\Gamma_{h}}=0$ ).

Corresponding to $\widetilde{A}_{h}(\cdot, \cdot)$ and $\widetilde{b}_{h}(\cdot, \cdot)$ in $(3.12)$ and (3.13) respectively, we define new continuous bilinear forms $A_{h}^{\mathrm{NI}}(\cdot, \cdot), b_{h}^{\mathrm{NI}}(\cdot, \cdot)$ with the help of numerical integration schemes $(3.14)-(3.15)$ satisfying $(\mathbf{A} 8)$ :

$$
\forall \Psi_{h}, \Phi_{h} \in \mathbf{V}_{h}, \quad A_{h}^{\mathrm{NI}}\left(\Phi_{h}, \Psi_{h}\right)=\sum_{T \in \tau_{h}^{\mathrm{ISO}}} \sum_{n=1}^{N_{1}} w_{n, T}^{1}\left(A_{i j k l} \phi_{h i j} \psi_{h k l}\right)\left(b_{n, T}^{1}\right)=A_{h}^{\mathrm{NI}}\left(\Psi_{h}, \Phi_{h}\right)
$$

with $\left|A_{h}^{\mathrm{NI}}\left(\Phi_{h}, \Psi_{h}\right)\right| \leq M_{0}\left\|\Psi_{h}\right\|_{0, \Omega_{h}}\left\|\Phi_{h}\right\|_{0, \Omega_{h}} \leq M_{0}\left\|\Psi_{h}\right\|_{1, \Omega_{h}}\left\|\Phi_{h}\right\|_{0, \Omega_{h}}$ for some $M_{0}>0$

$$
b_{h}^{\mathrm{NI}}\left(\Phi_{h}, v_{h}\right)=\sum_{T \in \tau_{h}^{\mathrm{ISO}}} \sum_{n=1}^{N_{0}} w_{n, T}^{0}\left(\phi_{h i j, j} v_{h, i}\right)\left(b_{n, T}^{0}\right) \quad \forall \Phi_{h} \in \mathbf{V}_{h}, \forall v_{h} \in W_{h}
$$

with $\left|b_{h}^{\mathrm{NI}}\left(\Phi_{h}, v_{h}\right)\right| \leq m_{0}\left\|\Phi_{h}\right\|_{1, \Omega_{h}}\left\|v_{h}\right\|_{1, \Omega_{h}}$ for some $m_{0}>0$. Now, we make the assumption:

(A9): $A_{h}^{\mathrm{NI}}(\cdot, \cdot) \quad$ in $(3.19) \quad\left(\right.$ resp. $\quad b_{h}^{\mathrm{NI}}(\cdot, \cdot)$ in $\left.(3.20)\right)$ is evaluated using quadrature scheme $(3.14)-(3.15)$ for $i=1$ (resp. $i=0$ ), which is exact for $P_{6}(\hat{T})$ resp. $\left.P_{3}(\hat{T})\right)$.

Now, to the eigenvalue problem $\left(\mathbf{Q}^{\mathbf{E}}\right)$, we associate the Isoparametric Mixed Finite Element Eigenvalue Problem $\left(\mathbf{Q}_{\mathbf{h}}^{\mathbf{E}}\right)$ :

$\forall h \in] 0, h_{0}$ [ with $h_{0}>0$, find $\lambda_{h} \in \mathbb{R}$ for which $\exists$ non-null $\left(\Psi_{h}, u_{h}\right) \in \mathbf{V}_{h} \times W_{h}$ such that

$$
\left(\mathbf{Q}_{\mathbf{h}}^{\mathbf{E}}\right): A_{h}^{\mathrm{NI}}\left(\Psi_{h}, \Phi_{h}\right)+b_{h}^{\mathrm{NI}}\left(\Phi_{h}, u_{h}\right)=0 \quad \forall \Phi_{h} \in \mathbf{V}_{h}, \quad-b_{h}^{\mathrm{NI}}\left(\Psi_{h}, v_{h}\right)=\lambda_{h}\left\langle u_{h}, v_{h}\right\rangle_{0, \Omega_{h}} \quad \forall v_{h} \in W_{h},
$$


and the associated Isoparametric Mixed Finite Element Source Problem $\left(\mathbf{Q}_{\mathbf{h}}\right)$, which corresponds to (Q) in (2.23) is defined by: For given $\tilde{f} \in L^{2}\left(\Omega_{h}\right)$, find $\left(\Psi_{h}, u_{h}\right) \in \mathbf{V}_{h} \times W_{h}$ such that

$$
\left(\mathbf{Q}_{\mathbf{h}}\right): A_{h}^{\mathrm{NI}}\left(\Psi_{h}, \Phi_{h}\right)+b_{h}^{\mathrm{NI}}\left(\Phi_{h}, u_{h}\right)=0 \quad \forall \Phi_{h} \in \mathbf{V}_{h}, \quad-b_{h}^{\mathrm{NI}}\left(\Psi_{h}, v_{h}\right)=\left\langle\tilde{f}, v_{h}\right\rangle_{0}, \Omega_{h} \quad \forall v_{h} \in W_{h},
$$

$$
\text { where }\left\langle\tilde{f}, v_{h}\right\rangle_{0, \Omega_{h}}=\int_{\Omega_{h}} \tilde{f} v_{h} \mathrm{~d} \Omega_{h} \quad \forall v_{h} \in W_{h} \text { with } \tilde{f} \in L^{2}(\widetilde{\Omega}), \tilde{f}=\tilde{f} \downarrow_{\Omega_{h}}
$$

(denoted by the same notation $\tilde{f}$ ), $\tilde{f}$ being the extension to $\widetilde{\Omega}$ of $f$ with $\tilde{f}=\Lambda \tilde{u}$ (see [10]). Now, based on Theorem 3.1 and Corollary 3.1, we define $\widetilde{X}_{h}, \widetilde{\mathbf{V}}_{h}$ and $\widetilde{W}_{h}$ :

$$
\begin{gathered}
\widetilde{X}_{h}=\left\{\widetilde{\phi}_{h}: \widetilde{\phi}_{h} \in H^{1}(\widetilde{\Omega}), \widetilde{\phi}_{h} \downarrow_{\Omega_{h}}=\phi_{h} \in X_{h}\right\} \text { with }\left\|\widetilde{\phi}_{h}\right\|_{1, \widetilde{\Omega}} \leq C\left\|\phi_{h}\right\|_{1, \Omega_{h}} \text { for some } C>0 ; \\
\widetilde{\mathbf{V}}_{h}=\left\{\widetilde{\Phi}_{h}: \widetilde{\Phi}_{h}=\left(\widetilde{\phi}_{h i j}\right)_{i, j=1,2} \text { with } \widetilde{\phi}_{h i j}=\widetilde{\phi}_{h j i} \in \widetilde{X}_{h} \forall i, j=1,2, \widetilde{\Phi}_{h} \downarrow_{\Omega_{h}}=\Phi_{h} \in \mathbf{V}_{h}\right\} \\
\text { with }\left\|\widetilde{\phi}_{h i j}\right\|_{1, \widetilde{\Omega}} \leq C\left\|\phi_{h i j}\right\|_{1, \Omega_{h}} \forall i, j=1,2,\left\|\widetilde{\Phi}_{h}\right\|_{1, \widetilde{\Omega}} \leq C\left\|\Phi_{h}\right\|_{1, \Omega_{h}} ; \\
\widetilde{W}_{h}=\left\{\widetilde{\chi}_{h}: \widetilde{\chi}_{h} \in H_{0}^{1}(\widetilde{\Omega}) \text { with } \widetilde{\chi} \downarrow_{\widetilde{\Omega} / \Omega_{h}}=0\right\} \text { with }\left\|\widetilde{\chi}_{h}\right\|_{1, \widetilde{\Omega}}=\left\|\chi_{h}\right\|_{1, \Omega_{h}} .
\end{gathered}
$$

Theorem 3.2 ( [10]). Let assumptions (A1-A9) hold. Then,

(i) $\exists \bar{\alpha}_{0}>$, independent of $h$, such that $A_{h}^{\mathrm{NI}}\left(\Phi_{h}, \Phi_{h}\right) \geq \bar{\alpha}_{0}\left\|\Phi_{h}\right\|_{0, \Omega_{h}}^{2} \forall \Phi_{h} \in \mathbf{V}_{h}$;

(ii) $\exists \bar{\beta}_{0}>0$, independent of $h$, such that $\sup _{\Phi_{h} \in \mathbf{V}_{h}-\{0\}} \frac{\left|b_{h}^{\mathrm{NI}}\left(\Phi_{h}, \chi_{h}\right)\right|}{\left\|\Phi_{h}\right\| \mathbf{V}_{h}} \geq \bar{\beta}_{0}\left\|\chi_{h}\right\|_{1, \Omega_{h}} \quad \forall \chi_{h} \in W_{h} \quad$ [10];

(iii) The isoparametric mixed finite element source problem $\left(\mathbf{Q}_{\mathbf{h}}\right)$ defined in (3.22) has a unique solution.

Remark 3.1. $\mathbf{V}_{h^{-}}$ellipticity of $A_{h}^{\mathrm{NI}}(\cdot, \cdot)$ in (3.28) will hold even if the quadrature scheme (3.14) with $\mathrm{i}=1$ be exact for $P_{4}(\hat{T})$ (instead of $P_{6}(\hat{T})$ in $(\mathbf{A 9})$ ) (see [10]). But this assumption (A9) will be necessary in Proposition 4.1 (see Rem. 5.4 for more details).

Hence, we can define $\overrightarrow{\mathbf{T}}_{\mathbf{h}}: \tilde{f} \in L^{2}\left(\Omega_{h}\right) \mapsto \overrightarrow{\mathbf{T}}_{\mathbf{h}} \tilde{f}=\left(S_{h} \tilde{f}, T_{h} \tilde{f}\right)=\left(\Psi_{h}, u_{h}\right) \in \mathbf{V}_{h} \times W_{h}$ such that

$$
A_{h}^{\mathrm{NI}}\left(S_{h} \tilde{f}, \Phi_{h}\right)+b_{h}^{\mathrm{NI}}\left(\Phi_{h}, T_{h} \tilde{f}\right)=0 \forall \Phi_{h} \in \mathbf{V}_{h} ;-b_{h}^{\mathrm{NI}}\left(S_{h} \tilde{f}, v_{h}\right)=\left\langle\tilde{f}, v_{h}\right\rangle_{0, \Omega_{h}} \forall v_{h} \in W_{h},
$$

where $S_{h} \in \mathcal{L}\left(L^{2}\left(\Omega_{h}\right) ; \mathbf{V}_{h}\right)$ and $T_{h} \in \mathcal{L}\left(L^{2}\left(\Omega_{h}\right) ; W_{h}\right)$ with $S_{h} \tilde{f}=\Psi_{h} \in \mathbf{V}_{h}, \quad T_{h} \tilde{f}=u_{h} \in W_{h}$ and $\left\|S_{h} \tilde{f}\right\|_{0, \Omega_{h}}+$ $\left\|T_{h} \tilde{f}\right\|_{1, \Omega_{h}} \leq C\|\tilde{f}\|_{0, \Omega_{h}}$ for some $C>0$, independent of h [8].

Theorem 3.3 ( [10]). Let assumptions (A1-A9) hold and $(\Psi, u) \in \mathbf{V} \times W$ with $u \in H^{3}(\Omega) \cap H_{0}^{2}(\Omega)$ (resp. $\left.\left(\Psi_{h}, u_{h}\right) \in \mathbf{V}_{h} \times W_{h}\right)$ be the unique solution of $(\mathbf{Q})\left(\right.$ resp. $\left.\left(\mathbf{Q}_{\mathbf{h}}\right)\right)$.

Let $\tilde{u} \in H^{3}(\widetilde{\Omega})$ be an extension to $\widetilde{\Omega}$ of $u \in H^{3}(\Omega) \cap H_{0}^{2}(\Omega)$ such that $\tilde{\psi}_{i j}=a_{i j k l} \tilde{u}_{, k l} \in H^{1}(\widetilde{\Omega}) \quad \forall i, j=1,2$ with $\tilde{\Psi}=\left(\tilde{\psi}_{i j}\right)_{1 \leq i, j \leq 2}$ and $(\tilde{\Psi}, \tilde{u}) \in \tilde{\mathbf{V}} \times \tilde{W}$ (resp. $\left.\left(\tilde{\Psi}_{h}, \tilde{u}_{h}\right) \in \tilde{\mathbf{V}}_{h} \times \tilde{W}_{h}\right)$ be an extension to $\widetilde{\Omega}$ of $(\Psi, u) \in$ $\mathbf{V} \times W$ (resp. $\left.\left(\Psi_{h}, u_{h}\right) \in \mathbf{V}_{h} \times W_{h}\right)$. Let $\tilde{f} \in L^{2}(\widetilde{\Omega})$ be an extension to $\widetilde{\Omega}$ of $f \in L^{2}(\Omega)$ such that $\tilde{f}=\Lambda \tilde{u}$. Then, $\exists C>0$, independent of $h$, such that

$$
\left\|\tilde{\Psi}-\Psi_{h}\right\|_{0, \Omega_{h}} \leq C h\left[\|\tilde{u}\|_{3, \tilde{\Omega}}+\|\tilde{\Psi}\|_{1, \tilde{\Omega}}\right], \quad\left\|\tilde{u}-u_{h}\right\|_{1, \Omega_{h}} \leq C h\left[\|\tilde{u}\|_{3, \tilde{\Omega}}+\|\tilde{\Psi}\|_{1, \tilde{\Omega}}\right] .
$$


Since $W_{h} \subset H_{0}^{1}\left(\Omega_{h}\right) \subset L^{2}\left(\Omega_{h}\right)$, we consider $T_{h} \downarrow_{W_{h}}=T_{h} \in \mathcal{L}\left(W_{h}\right)$ as the finite dimensional linear operator on $\left(W_{h} ;\langle\cdot, \cdot\rangle_{0, \Omega_{h}}\right)$ defined by: $T_{h}: \chi_{h} \in W_{h} \mapsto T_{h} \chi_{h}=w_{h} \in W_{h}$ such that $\forall \chi_{h} \in W_{h}$,

$$
A_{h}^{\mathrm{NI}}\left(S_{h} \chi_{h}, \Phi_{h}\right)+b_{h}^{\mathrm{NI}}\left(\Phi_{h}, T_{h} \chi_{h}\right)=0 \quad \forall \Phi_{h} \in \mathbf{V}_{h}, \quad-b_{h}^{\mathrm{NI}}\left(S_{h} \chi_{h}, v_{h}\right)=\left\langle\chi_{h}, v_{h}\right\rangle_{0, \Omega_{h}} \forall v_{h} \in W_{h} .
$$

Then, $\left(\mu_{h} ; u_{h}\right) \in \mathbb{R}^{+} \times W_{h}$ is an eigenpair of the symmetric, positive-definite operator $T_{h}$ on $W_{h} \Longleftrightarrow$ $\left(\lambda_{h} ;\left(\Psi_{h}, u_{h}\right)\right) \in \mathbb{R}^{+} \times\left(\mathbf{V}_{h} \times W_{h}\right)$ is an eigenpair of $\left(\mathbf{Q}_{\mathbf{h}}^{\mathbf{E}}\right)$ with $\lambda_{h}=1 / \mu_{h}$ and $\Psi_{h}=S_{h}\left(\lambda_{h} u_{h}\right)$, and we have:

Theorem 3.4 ( [8]). $\left(\mathbf{Q}_{\mathbf{h}}^{\mathbf{E}}\right)$ has strictly positive, possibly repeated, real eigenvalues: For $N_{h}=\operatorname{dim} W_{h}$, $0<\lambda_{1, h} \leq \lambda_{2, h} \leq \cdots \leq \lambda_{N_{h}, h}$ with $\lambda_{k, h}=1 / \mu_{k, h}, 1 \leq k \leq N_{h},\left(\mu_{1, h} \geq \mu_{2, h} \geq \cdots \geq \mu_{N_{h}, h}>0\right.$ being eigenvalues of $\left.T_{h}\right)$, and $\exists$ corresponding eigensolutions $\left(\Psi_{m, h}, u_{m, h}\right) \in \mathbf{V}_{h} \times W_{h}, 1 \leq m \leq N_{h}$, of $\left(\mathbf{Q}_{\mathbf{h}}^{\mathbf{E}}\right)$ i.e. $A_{h}^{\mathrm{NI}}\left(\Psi_{m, h}, \Phi_{h}\right)+b_{h}^{\mathrm{NI}}\left(\Phi_{h}, u_{m, h}\right)=0 \forall \Phi_{h} \in \mathbf{V}_{h},-b_{h}^{\mathrm{NI}}\left(\Psi_{m, h}, v_{h}\right)=\lambda_{m, h}\left\langle u_{m, h}, v_{h}\right\rangle_{0, \Omega_{h}} \forall v_{h} \in W_{h}$ such that $\left(u_{m, h}\right)_{m=1}^{N_{h}}$ is an orthonormal basis in $\left(W_{h},\langle\cdot, \cdot\rangle_{0, \Omega_{h}}\right)$ and $\left(\frac{\Psi_{m, h}}{\sqrt{\lambda_{m, h}}}\right)_{m=1}^{N_{h}}$ is an orthonormal system in $\left(\mathbf{V}_{h},[\cdot, \cdot]_{A_{h}^{\mathrm{NI}}(\cdot, \cdot)}\right)$; i.e. in $\mathbf{V}_{h}$ equipped with inner product $[\cdot, \cdot]_{A_{h}^{\mathrm{NI}}(\cdot, \cdot)}$.

By virtue of (3.28), applying Lax-Milgram lemma, we can define $\mathcal{I}_{h}: v_{h} \in W_{h} \mapsto \mathcal{I}_{h} v_{h}=\underline{\sigma}_{h} \in \mathbf{V}_{h}$ such that $A_{h}^{\mathrm{NI}}\left(\mathcal{I}_{h} v_{h}, \Phi_{h}\right)+b_{h}^{\mathrm{NI}}\left(\Phi_{h}, v_{h}\right)=0 \quad \forall \Phi_{h} \in \mathbf{V}_{h}$, and set

$$
\begin{aligned}
\mathcal{E}_{h}=\mathcal{I}_{h}\left(W_{h}\right) & =\left\{\underline{\sigma}_{h}: \underline{\sigma}_{h} \in \mathbf{V}_{h} \text { for which } \exists v_{h} \in W_{h}\right. \text { such that } \\
A_{h}^{\mathrm{NI}}\left(\underline{\sigma}_{h}, \Phi_{h}\right)+b_{h}^{\mathrm{NI}}\left(\Phi_{h}, v_{h}\right) & \left.=0 \forall \Phi_{h} \in \mathbf{V}_{h}\right\}=\operatorname{Span}\left\{\left(\frac{\Psi_{m, h}}{\sqrt{\lambda_{m, h}}}\right)_{m=1}^{N_{h}}\right\} .
\end{aligned}
$$

Then, $\mathcal{I}_{h}:\left(W_{h},\langle\cdot, \cdot\rangle_{0, \Omega_{h}}\right) \longrightarrow\left(\mathcal{E}_{h},[\cdot, \cdot]_{A_{h}^{\mathrm{NI}}(\cdot, \cdot)}\right)$ is linear and bijective. Then, $\left(\lambda_{m, h} ;\left(\Psi_{m, h}, u_{m, h}\right)\right) \in \mathbb{R}^{+} \times$ $\left(\mathbf{V}_{h} \times W_{h}\right)$ is an eigenpair of $\left(\mathbf{Q}_{\mathbf{h}}^{\mathbf{E}}\right) \Longrightarrow \Psi_{m, h}=\mathcal{I}_{h} u_{m, h}, 1 \leq m \leq N_{h}=\operatorname{dim} W_{h}$.

Define $N_{h^{-}}$dimensional space $\mathcal{M}_{h}$ of linked pairs $\left(\underline{\sigma}_{h}, v_{h}\right)=\left(\mathcal{I}_{h} v_{h}, v_{h}\right)$ by: $\mathcal{M}_{h}=\mathcal{E}_{h} \times W_{h}$. Then, $\left(\Psi_{m, h}, u_{m, h}\right) \in \mathcal{M}_{h}$ for $1 \leq m \leq N_{h}$.

Rayleigh quotient characterization of approximate eigenvalues.

As in the continuous case, $\forall$ linked pair $\left(\underline{\sigma}_{h}, v_{h}\right) \in \mathcal{M}_{h}$, we define the new Rayleigh quotient

$$
\Re_{h}(\cdot, \cdot) \text { by : } \Re_{h}\left(\underline{\sigma}_{h}, v_{h}\right)=\frac{A_{h}^{\mathrm{NI}}\left(\underline{\sigma}_{h}, \underline{\sigma}_{h}\right)}{\left\langle v_{h}, v_{h}\right\rangle_{0, \Omega_{h}}} \forall\left(\underline{\sigma}_{h}, v_{h}\right) \in \mathcal{M}_{h} .
$$

Define $\quad U_{p, h}=\operatorname{Span}\left\{\left(u_{m, h}\right)_{m=1}^{p}\right\} \subset W_{h}, \mathcal{M}_{p, h}=\operatorname{Span}\left\{\left(\Psi_{m, h}, u_{m, h}\right)_{m=1}^{p}\right\} \subset \mathcal{M}_{h}$,

where $\left(\lambda_{m, h},\left(\Psi_{m, h}, u_{m, h}\right)\right)_{m=1}^{p}$ are the first ' $p$ ' eigenpairs of $\left(\mathbf{Q}_{\mathbf{h}}^{\mathbf{E}}\right)$ with $0<\lambda_{1, h} \leq \lambda_{2, h} \leq \cdots \leq \lambda_{p, h}$ with $p \leq N_{h},\left\langle u_{m, h}, u_{n, h}\right\rangle_{0, \Omega_{h}}=\delta_{m n},\left[\frac{\Psi_{m, h}}{\sqrt{\lambda_{m, h}}}, \frac{\Psi_{n, h}}{\sqrt{\lambda_{n, h}}}\right]=\delta_{m n}$.

Theorem 3.5 (Min-Max Principle, [2,37]).

(i) Eigensolutions of $\left(\mathbf{Q}_{\mathbf{h}}^{\mathbf{E}}\right)$ are the stationary points of $\Re_{h}(\cdot, \cdot)$ on $\mathcal{M}_{h}$, the corresponding eigenvalues of $\left(\mathbf{Q}_{\mathbf{h}}^{\mathbf{E}}\right)$ being the values of $\Re_{h}(\cdot, \cdot)$ at these stationary points;

(ii) $\lambda_{p, h}=\min _{\substack{S_{p, h} \subset \mathcal{M}_{h} \\ \operatorname{dim} S_{p, h}=p}} \max _{\left(\sigma_{h}, v_{h}\right) \in S_{p, h}} \Re_{h}\left(\underline{\sigma}_{h}, v_{h}\right)=\max _{\left(\underline{\sigma}_{h}, v_{h}\right) \in \mathcal{M}_{p, h}} \Re_{h}\left(\underline{\sigma}_{h}, v_{h}\right)=\Re_{h}\left(\Psi_{p, h}, u_{p, h}\right) \forall p=1,2, \cdots, N_{h}$ $\left(\left(\lambda_{p, h},\left(\Psi_{p, h}, u_{p, h}\right)\right)\right.$ being an eigenpair of $\left.\left(\mathbf{Q}_{\mathbf{h}}^{\mathbf{E}}\right)\right)$. 
Since $T_{h} \in \mathcal{L}\left(W_{h}\right)$ is a symmetric, positive-definite, linear operator, we can define another

$$
\text { Rayleigh quotient } Q_{h}(\cdot) \text { by } Q_{h}\left(v_{h}\right)=\frac{\left\langle T_{h} v_{h}, v_{h}\right\rangle_{0, \Omega_{h}}}{\left\langle v_{h}, v_{h}\right\rangle_{0, \Omega_{h}}} \forall v_{h} \in W_{h} \text {. }
$$

Theorem 3.6 (Max-Min Principle, [2,37]). For $1 \leq p \leq N_{h}=\operatorname{dim} W_{h}$,

$$
\mu_{p, h}=\max _{\substack{S_{p, h}^{*} \subset W_{h} \\ \operatorname{dim} S_{p, h}^{*}=p}} \min _{v_{h} \in S_{p, h}^{*}} Q_{h}\left(v_{h}\right)=\min _{v_{h} \in U_{p, h} \subset W_{h}} Q_{h}\left(v_{h}\right)=Q_{h}\left(u_{p, h}\right),
$$

$\left(\mu_{p, h} ; u_{p, h}\right)$ being the $p$-th eigenpair of $T_{h}$ with $\mu_{p, h}=1 / \lambda_{p, h}$.

For $\chi_{p}^{*} \in H^{3}(\Omega) \cap H_{0}^{2}(\Omega)$ defined in $(2.26)$, let $\widetilde{\chi}_{p}^{*} \in H^{3}(\widetilde{\Omega})$ be its extension to $\widetilde{\Omega}$ and $\widetilde{\chi}_{p}^{*} \downarrow_{\Omega_{h}}$ be the restriction to $\Omega_{h}$ of $\tilde{\chi}_{p}^{*}$, which will be denoted by the same notation $\tilde{\chi}_{p}^{*}$, such that

$$
\left.\left\|\widetilde{\chi}_{p}^{*}\right\|_{3, \Omega_{h}} \leq C\left\|\tilde{\chi}_{p}^{*}\right\|_{3, \widetilde{\Omega}} \leq C\left\|\chi_{p}^{*}\right\|_{3, \Omega} \text { for some } C>0 \text { (see }(3.9)\right)
$$

Then, $\exists$ a unique $\overrightarrow{\mathbf{T}}_{\mathbf{h}} \widetilde{\chi}_{p}^{*}=\left(S_{h} \widetilde{\chi}_{p}^{*}, T_{h} \widetilde{\chi}_{p}^{*}\right) \in \mathbf{V}_{h} \times W_{h}$ defined by (3.30), i.e.

$$
A_{h}^{\mathrm{NI}}\left(S_{h} \tilde{\chi}_{p}^{*}, \Phi_{h}\right)+b_{h}^{\mathrm{NI}}\left(\Phi_{h}, T_{h} \tilde{\chi}_{p}^{*}\right)=0 \forall \Phi_{h} \in \mathbf{V}_{h} ;-b_{h}^{\mathrm{NI}}\left(S_{h} \tilde{\chi}_{p}^{*}, v_{h}\right)=\left\langle\widetilde{\chi}_{p}^{*}, v_{h}\right\rangle_{0, \Omega_{h}} \forall v_{h} \in W_{h}
$$

Since from $(2.26) \overrightarrow{\mathbf{T}} \chi_{p}^{*}=\left(\underline{\sigma}_{p}, \chi_{p}\right) \in \mathcal{M}_{p}$, it suggests to define a new linear operator $\vec{\Pi}_{\mathbf{h}}: \mathcal{M}_{p} \longrightarrow \mathcal{M}_{h} \subset \mathbf{V}_{h} \times W_{h}$ by: $\left(\underline{\sigma}_{p}, \chi_{p}\right) \in \mathcal{M}_{p} \mapsto \underline{\Pi}_{\mathbf{h}}\left(\underline{\sigma}_{p}, \chi_{p}\right)=\left(\underline{\Pi}_{1 h} \underline{\sigma}_{p}, \Pi_{2 h} \chi_{p}\right)=\overrightarrow{\mathbf{T}}_{h} \tilde{\chi}_{p}^{*}=\left(S_{h} \tilde{\chi}_{p}^{*}, T_{h} \tilde{\chi}_{p}^{*}\right)$ such that $\underline{\Pi}_{1 h} \underline{\sigma}_{p}=S_{h} \tilde{\chi}_{p}^{*}, \Pi_{2 h} \chi_{p}=T_{h} \tilde{\chi}_{p}^{*}$ i.e.

$$
A_{h}^{\mathrm{NI}}\left(\underline{\Pi}_{1 h} \underline{\sigma}_{p}, \Phi_{h}\right)+b_{h}^{\mathrm{NI}}\left(\Phi_{h}, \Pi_{2 h} \chi_{p}\right)=0 \forall \Phi_{h} \in \mathbf{V}_{h} ;-b_{h}^{\mathrm{NI}}\left(\underline{\Pi}_{1 h} \underline{\sigma}_{p}, v_{h}\right)=\left\langle\widetilde{\chi}_{p}^{*}, v_{h}\right\rangle_{0, \Omega_{h}} \forall v_{h} \in W_{h}
$$

Then we have: $\left(\underline{\Pi}_{\mathbf{h}} \cdot \overrightarrow{\mathbf{T}}\right) \chi_{p}^{*}=\left(\overrightarrow{\mathbf{T}}_{h} \cdot \rho_{h} \cdot E\right) \chi_{p}^{*}$ with $\rho_{h}\left(E \chi_{p}^{*}\right)=\rho_{h} \tilde{\chi}_{p}^{*}=\tilde{\chi}_{p}^{*} \downarrow_{\Omega_{h}}, \underline{\Pi}_{\mathbf{h}}$ being a linear operator, $E$ (resp. $\rho_{h}$ ) being the extension (resp. restriction) operator satisfying (3.38) (see also Cor. 3.1). Applying Theorem 3.3, we get the following result:

Corollary 3.2. Let assumptions $(\mathbf{A} \mathbf{1}-\mathbf{A 9})$ hold. Let $\widetilde{\chi}_{p}^{*} \in H^{3}(\widetilde{\Omega})$ be an extension to $\widetilde{\Omega}$ of $\chi_{p}^{*} \in H^{3}(\Omega) \cap H_{0}^{2}(\Omega)$ defined in (2.26) such that (3.38) holds and $\left(\underline{\sigma}_{p}, \chi_{p}\right) \in \mathcal{M}_{p}$ with $\chi_{p} \in H^{3}(\Omega) \cap H_{0}^{2}(\Omega)$ and $\left\|\chi_{p}\right\|_{0, \Omega}=1$ be defined by:

$$
A\left(\underline{\sigma}_{p}, \Phi\right)+b\left(\Phi, \chi_{p}\right)=0 \quad \forall \Phi \in \mathbf{V} ; \quad-b\left(\underline{\sigma}_{p}, v\right)=\left\langle\chi_{p}^{*}, v\right\rangle_{0, \Omega} \forall v \in W
$$

Let $\underline{\Pi}_{\mathbf{h}}\left(\underline{\sigma}_{p}, \chi_{p}\right)=\left(\underline{\Pi}_{1 h} \underline{\sigma}_{p}, \Pi_{2 h} \chi_{p}\right) \in \mathcal{M}_{h} \subset \mathbf{V}_{h} \times W_{h}$ be defined by (3.40).

Then, $\exists C>0$, independent of ' $h$ ', such that $\left\|\underline{\widetilde{\sigma}}_{p}-\underline{\Pi}_{1 h} \underline{\sigma}_{p}\right\|_{0, \Omega_{h}} \leq C h\left(\left\|\widetilde{\chi}_{p}\right\|_{3, \widetilde{\Omega}}+\left\|\underline{\widetilde{\sigma}}_{p}\right\|_{1, \widetilde{\Omega}}\right)$,

$$
\left\|\tilde{\chi}_{p}-\Pi_{2 h} \chi_{p}\right\|_{1, \Omega_{h}} \leq C h\left(\left\|\tilde{\chi}_{p}\right\|_{3, \widetilde{\Omega}}+\left\|\tilde{\sigma}_{p}\right\|_{1, \tilde{\Omega}}\right)
$$

where $\underline{\widetilde{\sigma}}_{p} \in \widetilde{\mathbf{V}}$ (resp. $\widetilde{\chi}_{p} \in H^{3}(\widetilde{\Omega})$ ) is the extension to $\widetilde{\Omega}$ of $\underline{\sigma}_{p} \in \mathbf{V}$ (resp. $\chi_{p} \in H^{3}(\Omega) \cap H_{0}^{2}(\Omega)$ ). 


\section{ERror estimates}

Here, we shall develop error estimates for the case of simple eigenvalues.

Theorem 4.1. Let assumptions (A1-A9) hold. Let $\left(\lambda_{p} ;\left(\Psi_{p}, u_{p}\right)\right) \in \mathbb{R}^{+} \times(\mathbf{V} \times W)$ with $u_{p} \in H^{3}(\Omega) \cap H_{0}^{2}(\Omega)$ (resp. $\left.\quad\left(\lambda_{p, h} ;\left(\Psi_{p, h}, u_{p, h}\right)\right) \in \mathbb{R}^{+} \times\left(\mathbf{V}_{h} \times W_{h}\right)\right)$ be an eigenpair of $\left(\mathbf{Q}^{\mathbf{E}}\right)$ (resp. $\left.\left(\mathbf{Q}_{\mathbf{h}}^{\mathbf{E}}\right)\right)$, $\lambda_{p}\left(\right.$ resp. $\left.\lambda_{p, h}\right)$ being a simple eigenvalue of $\left(\mathbf{Q}^{\mathbf{E}}\right)$ (resp. $\left.\left(\mathbf{Q}_{\mathbf{h}}^{\mathbf{E}}\right)\right)$ and $\left(\widetilde{\Psi}_{p}, \widetilde{u}_{p}\right) \in \widetilde{\mathbf{V}} \times H^{3}(\widetilde{\Omega})$ (resp. $\left.\left(\widetilde{\Psi}_{p, h}, \widetilde{u}_{p, h}\right) \in \widetilde{\mathbf{V}}_{\mathbf{h}} \times \widetilde{W}_{h}\right)$ be the extension to $\widetilde{\Omega}$ of the eigensolution $\left(\Psi_{p}, u_{p}\right)$ of $\left(\mathbf{Q}^{\mathbf{E}}\right)$ (resp. $\left(\Psi_{p, h}, u_{p, h}\right)$ of $\left.\left(\mathbf{Q}_{\mathbf{h}}^{\mathbf{E}}\right)\right), 1 \leq p \leq N_{h}=\operatorname{dim} W_{h}$, satisfying (3.7)-(3.9). Then, $\exists C>0$, independent of ' $h$ ' and ' $p$ ', such that

$$
\begin{gathered}
\left\|\widetilde{u}_{p}-u_{p, h}\right\|_{1, \Omega_{h}} \leq C\left[\left\|\widetilde{u}_{p}-\chi_{h}\right\|_{1, \Omega_{h}}+\left\|\widetilde{\Psi}_{p}-\Psi_{p, h}\right\|_{0, \Omega_{h}}+\sup _{\Phi_{h} \in \mathbf{V}_{h}-\{0\}} \frac{\left|b_{h}^{\mathrm{NI}}\left(\Phi_{h}, \chi_{h}\right)-{\widetilde{b_{h}}}_{(}\left(\Phi_{h}, \chi_{h}\right)\right|}{\left\|\Phi_{h}\right\|_{1, \Omega_{h}}}\right. \\
\quad+\sup _{\Phi_{h} \in \mathbf{V}_{h}-\{0\}} \frac{\left|A_{h}^{\mathrm{NI}}\left(\Psi_{p, h}, \Phi_{h}\right)-\widetilde{A_{h}}\left(\Psi_{p, h}, \Phi_{h}\right)\right|}{\left\|\Phi_{h}\right\|_{1, \Omega_{h}}}+\sup _{\Phi_{h} \in \mathbf{V}_{h}-\{0\}} \frac{\left|\tilde{A}_{h}\left(\widetilde{\Psi}_{p}, \Phi_{h}\right)-A\left(\Psi_{p}, \widetilde{\Phi}_{h}\right)\right|}{\left\|\Phi_{h}\right\|_{1, \Omega_{h}}} \\
\left.\quad+\sup _{\Phi_{h} \in \mathbf{V}_{h}-\{0\}} \frac{\left|\tilde{b}_{h}\left(\Phi_{h}, \widetilde{u}_{p}\right)-b\left(\widetilde{\Phi}_{h}, u_{p}\right)\right|}{\left\|\Phi_{h}\right\|_{1, \Omega_{h}}}\right] \quad \forall \chi_{h} \in W_{h},\left(\widetilde{\Phi}_{h} \in \widetilde{\mathbf{V}}_{h} \text { with } \widetilde{\Phi}_{h} \downarrow \Omega_{h}=\Phi_{h} \in \mathbf{V}_{h}\right) .
\end{gathered}
$$

Proof.

$$
\left\|\widetilde{u}_{p}-u_{p, h}\right\|_{1, \Omega_{h}} \leq\left\|\widetilde{u}_{p}-\chi_{h}\right\|_{1, \Omega_{h}}+\left\|\chi_{h}-u_{p, h}\right\|_{1, \Omega_{h}} \quad \forall \chi_{h} \in W_{h} .
$$

From (3.29), $\exists \bar{\beta}_{0}>0$, independent of ' $h$ ' and ' $p$ ', such that

$$
\left\|\chi_{h}-u_{p, h}\right\|_{1, \Omega_{h}} \leq \frac{1}{\bar{\beta}_{0}} \sup _{\Phi_{h} \in \mathbf{V}_{h}-\{0\}} \frac{\left|b_{h}^{\mathrm{NI}}\left(\Phi_{h}, \chi_{h}-u_{p, h}\right)\right|}{\left\|\Phi_{h}\right\|_{1, \Omega_{h}}} .
$$

But $b_{h}^{\mathrm{NI}}\left(\Phi_{h}, \chi_{h}-u_{p, h}\right)=\widetilde{b_{h}}\left(\Phi_{h}, \chi_{h}-\widetilde{u}_{p}\right)+\left[b_{h}^{\mathrm{NI}}\left(\Phi_{h}, \chi_{h}\right)-\widetilde{b_{h}}\left(\Phi_{h}, \chi_{h}\right)\right]+\left[\widetilde{b_{h}}\left(\Phi_{h}, \widetilde{u}_{p}\right)-b_{h}^{\mathrm{NI}}\left(\Phi_{h}, u_{p, h}\right)\right]$.

Now, using (2.20) (resp. (3.21)), we have

$$
\begin{aligned}
\left|b_{h}^{\mathrm{NI}}\left(\Phi_{h}, \chi_{h}-u_{p, h}\right)\right| \leq & \left|\tilde{b}_{h}\left(\Phi_{h}, \chi_{h}-\widetilde{u}_{p}\right)\right|+\left|\widetilde{A}_{h}\left(\widetilde{\Psi}_{p}-\Psi_{p, h}, \Phi_{h}\right)\right|+\left|b_{h}^{\mathrm{NI}}\left(\Phi_{h}, \chi_{h}\right)-\tilde{b}_{h}\left(\Phi_{h}, \chi_{h}\right)\right| \\
& +\left|\tilde{A}_{h}\left(\widetilde{\Psi}_{p}, \Phi_{h}\right)-A\left(\Psi_{p}, \widetilde{\Phi}_{h}\right)\right|+\left|A_{h}^{\mathrm{NI}}\left(\Psi_{p, h}, \Phi_{h}\right)-\tilde{A}_{h}\left(\Psi_{p, h}, \Phi_{h}\right)\right| \\
& +\left|\tilde{b}_{h}\left(\Phi_{h}, \widetilde{u}_{p}\right)-b\left(\widetilde{\Phi}_{h}, u_{p}\right)\right| \forall \chi_{h} \in W_{h}\left(\widetilde{\Phi}_{h} \in \widetilde{\mathbf{V}}_{h} \text { with } \widetilde{\Phi}_{h} \downarrow_{\Omega_{h}} \in \mathbf{V}_{h}\right) .
\end{aligned}
$$

Applying the continuity of $\tilde{A}_{h}(\cdot, \cdot)$ and $\widetilde{b_{h}}(\cdot, \cdot)$ in (4.4) and using it in (4.3) and (4.2), (4.1) follows.

Remark 4.1. In (4.1), the third and fourth terms on the right hand side are due to numerical integration and the fifth and sixth terms appear owing to the approximation of the boundary.

For finding estimates, we will need the following important results.

Lemma 4.1 ( [41]). Let $\Gamma$ be Lipschitz-continuous curved boundary of the convex domain $\Omega$, which is piecewise of $C^{k}$-class, $\left.k \geq 3 . \forall h \in\right] 0, h_{0}\left[\right.$ with $h_{0}>0$, let $\tau_{h}^{\mathrm{ISO}}$ be the quasi-uniform regular isoparametric triangulation of $\bar{\Omega}$ defined in (3.4) and $\widetilde{\Omega}$ be the domain satisfying (A2) and (A7). Let $\epsilon_{h}$ and $\omega_{h}$ be defined by (3.5) and (3.6) respectively. Then, $\exists C>0$, independent of $h$, such that

$$
\text { (a) } \quad\|\widetilde{v}\|_{0, \epsilon_{h}} \leq C h^{3 / 2}\|\widetilde{v}\|_{1, \widetilde{\Omega}} ; \quad\|v\|_{0, \omega_{h}} \leq C h^{3 / 2}\|\widetilde{v}\|_{1, \widetilde{\Omega}} \forall \widetilde{v} \in H^{1}(\widetilde{\Omega}) \text { with } \widetilde{v} \downarrow_{\Omega}=v .
$$


(b) Moreover, if $\tilde{u} \in H^{3}(\widetilde{\Omega})$ be the extension to $\widetilde{\Omega}$ of $u \in H^{3}(\Omega) \cap H_{0}^{2}(\Omega)$, we have

$$
\left\|\tilde{u}_{, i}\right\|_{0, \epsilon_{h}} \leq C h^{3}|\tilde{u}|_{2, \tilde{\Omega}} \text { and }\|u, i\|_{0, \omega_{h}} \leq C h^{3}|\tilde{u}|_{2, \widetilde{\Omega}}(i=1,2) .
$$

Inverse inequalities [16]: $\forall \phi_{h} \in X_{h}\left(\operatorname{resp} . \Phi_{h} \in \mathbf{V}_{h}\right), \exists C>0$,

$$
\left|\phi_{h}\right|_{1, \Omega_{h}} \leq(C / h)\left\|\phi_{h}\right\|_{0, \Omega_{h}} \quad\left(\text { resp. }\left|\Phi_{h}\right|_{1, \Omega_{h}} \leq(C / h)\left\|\Phi_{h}\right\|_{0, \Omega_{h}}\right) .
$$

Now, $\forall h \in] 0, h_{0}\left[\right.$ with $h_{0}>0$, we define $X_{h}$-interpolation operator $\mathcal{P}_{h}: H^{s}(\widetilde{\Omega}) \longrightarrow X_{h}$ :

$$
\text { For } \widetilde{\chi} \in H^{s}(\widetilde{\Omega}), s \geq 2, \mathcal{P}_{h} \widetilde{\chi} \in X_{h}, \mathcal{P}_{h} \widetilde{\chi}\left(a_{i, T}\right)=\widetilde{\chi}\left(a_{i, T}\right)=\chi\left(a_{i, T}\right), 1 \leq i \leq 6, \forall T \in \tau_{h}^{\mathrm{ISO}} .
$$

Then, $\left\|\widetilde{\chi}-\mathcal{P}_{h} \tilde{\chi}\right\|_{r, \Omega_{h}} \leq C h^{s-r}|\widetilde{\chi}|_{s, \Omega_{h}}(s \geq 2)$, and $\tilde{\chi} \in H^{3}(\widetilde{\Omega}), \widetilde{\chi} \downarrow_{\Gamma}=0 \quad\left(\operatorname{resp} . \tilde{\chi} \downarrow_{\Gamma_{h}}=0\right)$

$$
\Longrightarrow \mathcal{P}_{h} \tilde{\chi} \in W_{h} \text { with }\left\|\widetilde{\chi}-\mathcal{P}_{h} \widetilde{\chi}\right\|_{r, \Omega_{h}} \leq C h^{3-r}|\widetilde{\chi}|_{3, \Omega_{h}}(r=0,1) \quad[16] .
$$

We have the following results:

- Under (A7-A9). $\forall \Phi \in \mathbf{V}\left(\Omega_{h}\right) \exists$ a tensor-valued function $\Theta_{h} \in \mathbf{V}_{h}$ such that $\widetilde{b_{h}}\left(\Phi, \chi_{h}\right)=b_{h}^{\mathrm{NI}}\left(\Theta_{h}, \chi_{h}\right) \forall \chi_{h} \in$ $W_{h}$, and $\exists C>0$, independent of $\mathrm{h}$, such that

$$
\left\|\Phi-\Theta_{h}\right\|_{r, \Omega_{h}} \leq C h^{1-r}\|\Phi\|_{1, \Omega_{h}}(r=0,1) \quad[10] .
$$

- Let $\widetilde{f}$ be an extension to $\widetilde{\Omega}$ of $f \in L^{2}(\Omega)$ with $\widetilde{f}=\Lambda \widetilde{u}=\left(a_{i j k l} \widetilde{u}_{, k l}\right)_{, i j} \in L^{2}(\widetilde{\Omega})$. Let $\tilde{u} \in H^{3}(\widetilde{\Omega})$ be an extension to $\widetilde{\Omega}$ of the solution $u \in H^{3}(\Omega) \cap H_{0}^{2}(\Omega)$ of $\left(\mathbf{P}_{\mathbf{G}}\right)$ such that $\widetilde{\psi}_{i j}=a_{i j k l} \widetilde{u}_{, k l} \in H^{1}(\widetilde{\Omega}) \quad \forall i, j=1,2$ and $\widetilde{\Psi}=\left(\widetilde{\psi}_{i j}\right)_{1 \leq i, j \leq 2} \in \widetilde{\mathbf{V}}$ with $\widetilde{u} \downarrow_{\Omega}=u \in H^{3}(\Omega) \cap H_{0}^{2}(\Omega), \widetilde{\Psi} \downarrow_{\Omega}=\Psi \in \mathbf{V}, \quad \widetilde{\Psi} \downarrow_{\Omega_{h}} \in \mathbf{V}\left(\Omega_{h}\right)$. Then, for $\widetilde{\Psi}, \exists \Theta_{h} \in \mathbf{V}_{h}$ such that $b_{h}^{\mathrm{NI}}\left(\Theta_{h}, \chi_{h}\right)=-\left\langle\tilde{f}, \chi_{h}\right\rangle_{0, \Omega_{h}}$ and

$$
\left\|\widetilde{\Psi}-\Theta_{h}\right\|_{r, \Omega_{h}} \leq C h^{1-r}\|\widetilde{\Psi}\|_{1, \Omega_{h}}(r=0,1) \text { for some } \mathrm{C}>0 \quad[10] .
$$

Proposition 4.1. Suppose that (A5) holds i.e. coefficients $A_{i j k l} \in W^{2, \infty}(\widetilde{\Omega}) \forall i, j, k, l=1,2$. Let assumptions (A1-A9) hold. Then, $\exists C>0$, independent of $h$, such that $\forall \Phi_{h}, \underline{\sigma}_{h} \in \mathbf{V}_{h}$,

$$
\left|\tilde{A}_{h}\left(\Phi_{h}, \underline{\sigma}_{h}\right)-A_{h}^{\mathrm{NI}}\left(\Phi_{h}, \underline{\sigma}_{h}\right)\right| \leq C h^{2}\|A\|_{2, \infty, \tilde{\Omega}}\left\|\Phi_{h}\right\|_{0, \Omega_{h}}\left\|\underline{\sigma}_{h}\right\|_{0, \Omega_{h}}
$$

where $\|A\|_{2, \infty, \widetilde{\Omega}} \geq\|A\|_{2, \infty, \Omega_{h}}=\sum_{T \in \tau_{h}^{\mathrm{ISO}}} \sum_{i, j, k, l=1}^{2}\left\|A_{i j k l}\right\|_{2, \infty, T}$.

Proof. The proof is similar to that given in [10] for $A_{i j k l} \in W^{1, \infty}(\widetilde{\Omega})$.

Remark 4.2. (4.12) gives an estimate of the error due to numerical integration associated with the definition of $A_{h}^{\mathrm{NI}}(\cdot, \cdot)$ in (3.19) (see also Rem. 4.1).

Proposition 4.2. Suppose that assumptions (A1-A9) hold. Let $\left(\lambda_{p} ;\left(\Psi_{p}, u_{p}\right)\right) \in \mathbb{R}^{+} \times(\mathbf{V} \times W)$ be an eigenpair of $\left(\mathbf{Q}^{\mathbf{E}}\right)$ corresponding to the simple eigenvalue $\lambda_{p}$ with $u_{p} \in H^{3}(\Omega) \cap H_{0}^{2}(\Omega), \Psi_{p}=\left(\psi_{p i j}\right)_{1 \leq i, j \leq 2}, \quad \psi_{p i j}=$ $a_{i j k l} u_{p, k l} \in H^{1}(\Omega) \quad \forall i, j=1,2$. Let $\widetilde{u}_{p} \in H^{3}(\widetilde{\Omega})$ be the extension to $\widetilde{\Omega}$ of $u_{p} \in H^{3}(\Omega) \cap H_{0}^{2}(\Omega)$ such that $\widetilde{\psi}_{p i j}=a_{i j k l} \widetilde{u}_{p, k l} \in H^{1}(\widetilde{\Omega}) \forall i, j=1,2, \widetilde{\Psi}_{p}=\left(\widetilde{\psi}_{p i j}\right)_{1 \leq i, j \leq 2} \in \widetilde{\mathbf{V}}$, and let $\widetilde{\Phi}_{h} \in \widetilde{\mathbf{V}}_{h}$ be an extension to $\widetilde{\Omega}$ of $\Phi_{h} \in \mathbf{V}_{h}$ defined in (3.25). Then, the following estimates hold:

$$
\begin{gathered}
\text { I. }\left|A\left(\Psi_{p}, \Psi_{p}\right)-\tilde{A}_{h}\left(\widetilde{\Psi}_{p}, \widetilde{\Psi}_{p}\right)\right| \leq C h^{3}\left\|\widetilde{u}_{p}\right\|_{3, \widetilde{\Omega}}\left\|\widetilde{\Psi}_{p}\right\|_{1, \widetilde{\Omega}} ; \quad \mathbf{I I} .\left|A\left(\Psi_{p}, \widetilde{\Phi}_{h}\right)-\widetilde{A}_{h}\left(\widetilde{\Psi}_{p}, \Phi_{h}\right)\right| \leq C h^{3}\left\|\widetilde{u}_{p}\right\|_{3, \widetilde{\Omega}}\left\|\Phi_{h}\right\|_{1, \Omega_{h}} ; \\
\text { III. }\left|b\left(\tilde{\Phi}_{h}, u_{p}\right)-\widetilde{b}_{h}\left(\Phi_{h}, \widetilde{u}_{p}\right)\right| \leq C h^{3}\left\|\widetilde{u}_{p}\right\|_{2, \widetilde{\Omega}}\left\|\Phi_{h}\right\|_{1, \Omega_{h}} ; \quad \mathbf{I V} .\left|\widetilde{b}_{h}\left(\Phi_{h}, \chi_{h}\right)-b_{h}^{\mathrm{NI}}\left(\Phi_{h}, \chi_{h}\right)\right| \leq C h^{2}\left\|\Phi_{h}\right\|_{1, \Omega_{h}}\left\|\chi_{h}\right\|_{1, \Omega_{h}} .
\end{gathered}
$$


Following [37], we prepare some new results to be used in the sequel.

Proposition 4.3. Let $\left(\underline{\sigma}_{p}, \chi_{p}\right) \in \mathcal{M}_{p}$ be a linked pair with $\chi_{p} \in H^{3}(\Omega) \cap H_{0}^{2}(\Omega),\left\|\chi_{p}\right\|_{0, \Omega}=1$ and $\underline{\sigma}_{p} \in \mathbf{V}$ be defined by (3.41) and $\widetilde{\chi}_{p} \in H^{3}(\widetilde{\Omega})$ (resp. $\underline{\widetilde{\sigma}}_{p} \in \widetilde{\mathbf{V}}$ ) be extension to $\widetilde{\Omega}$ of $\chi_{p}$ (resp. $\underline{\sigma}_{p}$ ). Let $\underline{\underline{\Pi}}_{\mathbf{h}}\left(\underline{\sigma}_{p}, \chi_{p}\right)=$ $\left(\underline{\Pi}_{1 h} \underline{\sigma}_{p}, \Pi_{2 h} \chi_{p}\right) \in \mathcal{M}_{h} \subset \mathbf{V}_{h} \times W_{h}$ be defined by (3.40) such that the estimates (3.42) hold. Then, $\left.\exists h_{0} \in\right] 0,1[$ such that $\forall h \in] 0, h_{0}[$

$$
\begin{array}{r}
\left\langle\Pi_{2 h} \chi_{p}, \Pi_{2 h} \chi_{p}\right\rangle_{0, \Omega_{h}}^{-1}<\left(1+2\left|\widetilde{\alpha}_{p, h}\right|\right), \quad \text { where } \widetilde{\alpha}_{p, h}=\left\|\chi_{p}\right\|_{0, \omega_{h}}^{2}-\left\|\widetilde{\chi}_{p}\right\|_{0, \epsilon_{h}}^{2}+\alpha_{p, h} \text { with } \\
\alpha_{p, h}=\max _{\left(\underline{\sigma}_{p}, \chi_{p}\right) \in \mathcal{M}_{p},\left\|\chi_{p}\right\|_{0, \Omega}=1}\left\{\left|2\left\langle\widetilde{\chi}_{p}, \widetilde{\chi}_{p}-\Pi_{2 h} \chi_{p}\right\rangle_{0, \Omega_{h}}-\left\|\widetilde{\chi}_{p}-\Pi_{2 h} \chi_{p}\right\|_{0, \Omega_{h}}^{2}\right|\right\} .
\end{array}
$$

Proof.

$$
\left\langle\Pi_{2 h} \chi_{p}, \Pi_{2 h} \chi_{p}\right\rangle_{0, \Omega_{h}}=\left\|\chi_{p}\right\|_{0, \Omega}^{2}-\left\|\chi_{p}\right\|_{0, \omega_{h}}^{2}+\left\|\widetilde{\chi}_{p}\right\|_{0, \epsilon_{h}}^{2}-\left[2\left\langle\widetilde{\chi}_{p}, \widetilde{\chi}_{p}-\Pi_{2 h} \chi_{p}\right\rangle_{0, \Omega_{h}}-\left\|\widetilde{\chi}_{p}-\Pi_{2 h} \chi_{p}\right\|_{0, \Omega_{h}}^{2}\right] .
$$

Also, from (3.42) and (4.14),

$$
\begin{gathered}
\alpha_{p, h} \leq C h \max _{\left(\underline{\sigma}_{p}, \chi_{p}\right) \in \mathcal{M}_{p},\left\|\chi_{p}\right\|_{0, \Omega}=1}\left\{\left(\left\|\widetilde{\chi}_{p}\right\|_{3, \widetilde{\Omega}}+\left\|\underline{\widetilde{\sigma}}_{p}\right\|_{1, \widetilde{\Omega}}\right)\left[2\left\|\tilde{\chi}_{p}\right\|_{0, \widetilde{\Omega}}+h\left(\left\|\widetilde{\chi}_{p}\right\|_{3, \widetilde{\Omega}}+\left\|\underline{\widetilde{\sigma}}_{p}\right\|_{1, \widetilde{\Omega}}\right)\right]\right\} \longrightarrow 0 \\
\text { as } \left.h \longrightarrow 0 \text { and } \exists h_{0} \in\right] 0,1[\text { such that } \forall h \in] 0, h_{0}\left[, \quad \alpha_{p, h}<1 / 4 .\right.
\end{gathered}
$$

Again, from (4.5), we have : $\left\|\chi_{p}\right\|_{0, \omega_{h}} \leq C h^{3 / 2}\left\|\widetilde{\chi}_{p}\right\|_{1, \widetilde{\Omega}},\left\|\widetilde{\chi}_{p}\right\|_{0, \epsilon_{h}} \leq C h^{3 / 2}\left\|\widetilde{\chi}_{p}\right\|_{1, \widetilde{\Omega}} \forall \widetilde{\chi}_{p} \in H^{3}(\widetilde{\Omega})$

with $\chi_{p} \in H^{3}(\Omega) \cap H_{0}^{2}(\Omega),\left\|\chi_{p}\right\|_{0, \Omega}=1$ and $\left(\underline{\sigma}_{p}, \chi_{p}\right) \in \mathcal{M}_{p}$. Then, the right hand sides of these two inequalities in (4.17) tend to 0 as $h \longrightarrow 0$. Hence, $\left.\exists h_{0} \in\right] 0,1[$ such that

$$
\forall h \in] 0, h_{0}\left[, \quad\left\|\chi_{p}\right\|_{0, \omega_{h}}<1 / 2,\left\|\widetilde{\chi}_{p}\right\|_{0, \epsilon_{h}}<1 / \sqrt{2} .\right.
$$

Thus, $\left.\exists h_{0} \in\right] 0,1[$ such that $\forall h \in] 0, h_{0}[$,

$$
0 \leq\left\|\chi_{p}\right\|_{0, \omega_{h}}^{2}<1 / 4,0 \leq\left\|\tilde{\chi}_{p}\right\|_{0, \epsilon_{h}}^{2}<1 / 2,0 \leq \alpha_{p, h}<1 / 4 \Longrightarrow\left|\tilde{\alpha}_{p, h}\right|<1 / 2, \text { and }\left(1+2\left|\tilde{\alpha}_{p, h}\right|\right)<2 .
$$

Thus, from (4.15) and (4.19), $\forall h \in] 0, h_{0}\left[, h_{0} \in\right] 0,1[$, $\left\langle\Pi_{2 h} \chi_{p}, \Pi_{2 h} \chi_{p}\right\rangle_{0, \Omega_{h}} \geq 1-\left(\left\|\chi_{p}\right\|_{0, \omega_{h}}^{2}-\left\|\widetilde{\chi}_{p}\right\|_{0, \epsilon_{h}}^{2}+\alpha_{p, h}\right) \geq\left(1-\left|\widetilde{\alpha}_{p, h}\right|\right) \Longrightarrow\left\langle\Pi_{2 h} \chi_{p}, \Pi_{2 h} \chi_{p}\right\rangle_{0, \Omega_{h}}^{-1}<\left(1+2\left|\widetilde{\alpha}_{p, h}\right|\right)$. (In (4.16), (4.18) and (4.19), the same $\left.h_{0} \in\right] 0,1[$ has been used to denote different small positive numbers on $] 0,1\left[\right.$ and this convention of using the same $h_{0}$ to denote different small numbers on ] $0,1[$ at different steps will be followed also in the sequel).

Lemma 4.2. : $\forall h \in] 0, h_{0}\left[\right.$ with $\left.h_{0} \in\right] 0,1\left[\right.$ for which (4.19) holds, (i) linear operator $\underline{\Pi}_{\mathbf{h}}: \mathcal{M}_{p} \longrightarrow \underline{\Pi}_{\mathbf{h}} \mathcal{M}_{p} \subset$ $\mathcal{M}_{h}$ defined by (3.40) is injective, (ii) $\operatorname{dim}\left(\underline{\Pi}_{\mathbf{h}} \mathcal{M}_{p}\right)=\operatorname{dim} \mathcal{M}_{p}=p$.

Proof. For (i), we are to show that $\underline{\Pi}_{\mathbf{h}}\left(\underline{\sigma}_{p}, \chi_{p}\right)=\left(\underline{\Pi}_{1 h} \underline{\sigma}_{p}, \Pi_{2 h} \chi_{p}\right)=(\mathbf{0}, 0) \Longrightarrow\left(\underline{\sigma}_{p}, \chi_{p}\right)=(\mathbf{0}, 0)$. Assume the contrary, i.e. $\exists\left(\widehat{\sigma}_{p}, \widehat{\chi}_{p}\right) \neq(\mathbf{0}, 0)$ in $\mathcal{M}_{p}$ with $\widehat{\chi}_{p} \in H^{3}(\Omega) \cap H_{0}^{2}(\Omega)$ and $\left\|\widehat{\chi}_{p}\right\|_{0, \Omega}=1$, for which (4.19) holds 
$\forall h \in] 0, h_{0}\left[\right.$ with $h_{0}>0$, and $\underline{\underline{\Pi}}_{\mathbf{h}}\left(\underline{\widehat{\widehat{\sigma}}}_{p}, \widehat{\chi}_{p}\right)=\left(\underline{\Pi}_{1 h} \underline{\widehat{\underline{\sigma}}}_{p}, \Pi_{2 h} \widehat{\chi}_{p}\right)=(\mathbf{0}, 0)$ i.e. $\underline{\Pi}_{1 h} \widehat{\widehat{\sigma}}_{p}=\mathbf{0}, \Pi_{2 h} \widehat{\chi}_{p}=0$ such that $\widetilde{\widehat{\chi}}_{p} \in H^{3}(\widetilde{\Omega})$ is its extension to $\widetilde{\Omega}$.

$$
\begin{aligned}
\alpha_{p, h} & \geq\left\{\mid 2\left(\widetilde{\widehat{\chi}}_{p}, \widetilde{\widehat{\chi}}_{p}-\Pi_{2 h} \widehat{\chi}_{p}>_{0, \Omega_{h}}-\left\langle\widetilde{\widehat{\chi}}_{p}-\Pi_{2 h} \widehat{\chi}_{p}, \widetilde{\widehat{\chi}}_{p}-\Pi_{2 h} \widehat{\chi}_{p}\right\rangle_{0, \Omega_{h}} \mid\right\}\right. \text { (see (4.14)) } \\
& =1-\left\|\widehat{\chi}_{p}\right\|_{0, \omega_{h}}^{2}+\left\|\widetilde{\widehat{\chi}}_{p}\right\|_{0, \epsilon_{h}}^{2}
\end{aligned}
$$

with $\omega_{h}=\Omega-\left(\Omega \cap \Omega_{h}\right), \epsilon_{h}=\Omega_{h}-\left(\Omega \cap \Omega_{h}\right) \Longrightarrow \alpha_{p, h}+\left\|\widehat{\chi}_{p}\right\|_{0, \omega_{h}}^{2}-\left\|\widetilde{\widehat{\chi}}_{p}\right\|_{0, \epsilon_{h}}^{2} \geq 1$, which contradicts the hypothesis that (4.19) holds: $\forall h \in] 0, h_{0}\left[\right.$ with $\left.h_{0} \in\right] 0,1\left[, \alpha_{p, h}+\left\|\widehat{\chi}_{p}\right\|_{0, \omega_{h}}^{2}-\left\|\widetilde{\chi}_{p}\right\|_{0, \epsilon_{h}}^{2}<1 / 2\right.$. Hence, our assumption that $\exists\left(\widehat{\widehat{\underline{\sigma}}}_{p}, \widehat{\chi}_{p}\right) \neq(\mathbf{0}, 0)$ is wrong i.e. $\left(\underline{\widehat{\sigma}}_{p}, \widehat{\chi}_{p}\right)=(\mathbf{0}, 0) \Longrightarrow$ Linear operator $\underline{\underline{\Pi}}_{\mathbf{h}}$ is injective.

(ii) $\underline{\underline{\Pi}}_{\mathbf{h}}$ is linear and injective from p-dimensional space $\mathcal{M}_{p}$ onto $\underline{\vec{\Pi}}_{\mathbf{h}} \mathcal{M}_{p} \subset \mathcal{M}_{h} \Longrightarrow \operatorname{dim}\left(\underline{\vec{\Pi}}_{\mathbf{h}} \mathcal{M}_{p}\right)=\operatorname{dim} \mathcal{M}_{p}=p$.

Now, first of all, we will prove that $\lim _{h \rightarrow 0} \lambda_{p, h}=\lambda_{p}, \lambda_{p}$ (resp. $\lambda_{p, h}$ ) being a simple eigenvalue of $\left(\mathbf{Q}^{\mathbf{E}}\right)$ (resp. $\left.\left(\mathbf{Q}_{\mathbf{h}}^{\mathbf{E}}\right)\right)$, and using this, we will find the estimate for $\left\|\widetilde{u}_{p}-u_{p, h}\right\|_{0, \Omega_{h}}$ in order to find the "optimal" estimate for $\left|\lambda_{p}-\lambda_{p, h}\right|$ and finally, for $\left\|\widetilde{\Psi}_{p}-\Psi_{p, h}\right\|_{0, \Omega_{h}}$ and $\left\|\widetilde{u}_{p}-u_{p, h}\right\|_{1, \Omega_{h}}$ in this order (see also [14]). The proofs are highly technical in nature. For the sake of brevity, we state the outline of the proof and the final results (for details of proofs, see [8]).

Theorem 4.2. Let assumptions (A1-A9) and assumptions of Proposition 4.2 hold. Let $\underline{\Pi}_{\mathbf{h}}$ be the bijective operator defined by (3.40) such that Lemma 4.2 and estimates (3.42) hold $\forall h \in] 0, h_{0}\left[\right.$. Then, $\lim _{h \rightarrow 0} \lambda_{p, h}=\lambda_{p}$.

Proof. From Theorem 3.5,

$$
\begin{aligned}
& \lambda_{p, h}=\min _{\substack{S_{p, h} \subset \mathcal{M}_{h} \\
\operatorname{dim} S_{p, h}=p}} \max _{\left(\underline{\sigma}_{h}, v_{h}\right) \in S_{p, h}}\left[\frac{A_{h}^{\mathrm{NI}}\left(\underline{\sigma}_{h}, \underline{\sigma}_{h}\right)}{\left\langle v_{h}, v_{h}\right\rangle_{0, \Omega_{h}}}\right] \leq \max _{\left(\underline{\sigma}_{h}, v_{h}\right) \in \underline{\underline{\underline{H}}}_{\mathbf{h}} \mathcal{M}_{p}}\left[\frac{A_{h}^{\mathrm{NI}}\left(\underline{\sigma}_{h}, \underline{\sigma}_{h}\right)}{\left\langle v_{h}, v_{h}\right\rangle_{0, \Omega_{h}}}\right] \\
& \Longrightarrow \lambda_{p, h} \leq \max _{\substack{\left(\sigma_{p}, \chi_{p}\right) \in \mathcal{M}_{p} \\
\left\|\chi_{p}\right\|_{0, \Omega}=1}}\left[\frac{A_{h}^{\mathrm{NI}}\left(\underline{\Pi}_{1 h} \underline{\sigma}_{p}, \underline{\Pi}_{1 h} \underline{\sigma}_{p}\right)}{\left\langle\Pi_{2 h} \chi_{p}, \Pi_{2 h} \chi_{p}\right\rangle_{0, \Omega_{h}}}\right] \text {, since } \underline{\vec{\Pi}}_{\mathbf{h}}\left(\underline{\sigma}_{p}, \chi_{p}\right)=\left(\underline{\Pi}_{1 h} \underline{\sigma}_{p}, \Pi_{2 h} \chi_{p}\right) \in \underline{\vec{\Pi}}_{\mathbf{h}} \mathcal{M}_{p} .
\end{aligned}
$$

Then $\forall h \in] 0, h_{0}\left[\right.$ with some $\left.h_{0} \in\right] 0,1\left[\right.$, for $\left(\underline{\sigma}_{p}, \chi_{p}\right) \in \mathcal{M}_{p}$ with $\left\|\chi_{p}\right\|_{0, \Omega}=1$,

$$
\begin{aligned}
& \left\langle\Pi_{2 h} \chi_{p}, \Pi_{2 h} \chi_{p}>_{0, \Omega_{h}}^{-1}<1+2\left|\widetilde{\alpha}_{p, h}\right|<2 \quad(\text { see (4.13) and (4.19)) }\right. \\
& A_{h}^{\mathrm{NI}}\left(\underline{\Pi}_{1 h} \underline{\sigma}_{p}, \underline{\Pi}_{1 h} \underline{\sigma}_{p}\right) \leq A\left(\underline{\sigma}_{p}, \underline{\sigma}_{p}\right)+\left|\tilde{A}_{h}\left(\underline{\tilde{\sigma}}_{p}, \underline{\tilde{\sigma}}_{p}\right)-A\left(\underline{\sigma}_{p}, \underline{\sigma}_{p}\right)\right|+\left|\tilde{A}_{h}\left(\underline{\Pi}_{1 h} \underline{\sigma}_{p}, \underline{\Pi}_{1 h} \underline{\sigma}_{p}\right)-\tilde{\tilde{A}}_{h}\left(\underline{\widetilde{\sigma}}_{p}, \underline{\widetilde{\sigma}}_{p}\right)\right| \\
& +\left|A_{h}^{\mathrm{NI}}\left(\underline{\Pi}_{1 h} \underline{\sigma}_{p}, \underline{\Pi}_{1 h} \underline{\sigma}_{p}\right)-\tilde{A}_{h}\left(\underline{\Pi}_{1 h} \underline{\sigma}_{p}, \underline{\Pi}_{1 h} \underline{\sigma}_{p}\right)\right| \text { with } \underline{\tilde{\sigma}}_{p} \in \tilde{\mathbf{V}},\left(\underline{\sigma}_{p}, \chi_{p}\right) \in \mathcal{M}_{p} \text {, } \\
& \text { where - } A\left(\underline{\sigma}_{p}, \underline{\sigma}_{p}\right) \leq \max _{\substack{\left(\bar{\sigma}_{p}, \bar{\chi}_{p}\right) \in \mathcal{M}_{p} \\
\left\|\bar{\chi}_{p}\right\|_{0, \Omega}=1}}\left[\frac{A\left(\bar{\sigma}_{p}, \bar{\sigma}_{p}\right)}{\left\langle\bar{\chi}_{p}, \bar{\chi}_{p}\right\rangle_{0, \Omega}}\right]=\lambda_{p} \text { for linked pair }\left(\underline{\sigma}_{p}, \chi_{p}\right) \in \mathcal{M}_{p} \text {; }
\end{aligned}
$$


- $\left|\tilde{A}_{h}\left(\underline{\widetilde{\sigma}}_{p}, \underline{\widetilde{\sigma}}_{p}\right)-A\left(\underline{\sigma}_{p}, \underline{\sigma}_{p}\right)\right| \leq C h^{3}\left\|\underline{\widetilde{\sigma}}_{p}\right\|_{1, \tilde{\Omega}}\left\|\widetilde{\chi}_{p}\right\|_{3, \tilde{\Omega}} ; \quad$ (see (I), Prop. 4.2)

- $\left|\tilde{A}_{h}\left(\underline{\Pi}_{1 h} \underline{\sigma}_{p}, \underline{\Pi}_{1 h} \underline{\sigma}_{p}\right)-\tilde{A}_{h}\left(\underline{\widetilde{\sigma}}_{p}, \widetilde{\widetilde{\sigma}}_{p}\right)\right|=\left|\tilde{A}_{h}\left(\underline{\widetilde{\sigma}}_{p}-\underline{\Pi}_{1 h} \underline{\sigma}_{p}, \widetilde{\widetilde{\sigma}}_{p}-\underline{\Pi}_{1 h} \underline{\sigma}_{p}\right)-2 \tilde{A}_{h}\left(\underline{\widetilde{\sigma}}_{p}, \underline{\widetilde{\sigma}}_{p}-\underline{\Pi}_{1 h} \underline{\sigma}_{p}\right)\right|$

$\leq C h\left(\left\|\widetilde{\underline{\sigma}}_{p}\right\|_{1, \widetilde{\Omega}}+\left\|\widetilde{\chi}_{p}\right\|_{3, \widetilde{\Omega}}\right)\left[h\left(\left\|\widetilde{\widetilde{\sigma}}_{p}\right\|_{1, \widetilde{\Omega}}+\left\|\tilde{\chi}_{p}\right\|_{3, \widetilde{\Omega}}\right)+\left\|\underline{\widetilde{\sigma}}_{p}\right\|_{0, \tilde{\Omega}}\right]$

(using continuity of $\tilde{A}_{h}(\cdot, \cdot)$ and $(3.42)$ );

- $\left|A_{h}^{\mathrm{NI}}\left(\underline{\Pi}_{1 h} \underline{\sigma}_{p}, \underline{\Pi}_{1 h} \underline{\sigma}_{p}\right)-\tilde{A}_{h}\left(\underline{\Pi}_{1 h} \underline{\sigma}_{p}, \underline{\Pi}_{1 h} \underline{\sigma}_{p}\right)\right| \leq C h^{2}\|\widetilde{A}\|_{2, \infty, \tilde{\Omega}}\left[(1+h)\left\|\underline{\widetilde{\sigma}}_{p}\right\|_{1, \widetilde{\Omega}}+h\left\|\widetilde{\chi}_{p}\right\|_{3, \widetilde{\Omega}}\right]^{2}$,

from Proposition 4.1 and $\left\|\underline{\Pi}_{1 h} \underline{\sigma}_{p}\right\|_{0, \Omega_{h}} \leq\left\|\underline{\widetilde{\sigma}}_{p}\right\|_{0, \Omega_{h}}+\left\|\underline{\widetilde{\sigma}}_{p}-\underline{\Pi}_{1 h} \underline{\sigma}_{p}\right\|_{0, \Omega_{h}} \leq C\left[(1+h)\left\|\underline{\widetilde{\sigma}}_{p}\right\|_{1, \widetilde{\Omega}}+h\left\|\tilde{\chi}_{p}\right\|_{3, \widetilde{\Omega}}\right]$.

Hence, from (4.22)-(4.28),

$$
\begin{aligned}
\lambda_{p, h} \leq & \max _{\substack{\left(\underline{\underline{\sigma}}, \chi_{p} \in \mathcal{M}_{p} \\
\left\|\chi_{p}\right\|_{0, \Omega}=1\right.}}\left[\frac{A_{h}^{\mathrm{NI}}\left(\underline{\Pi}_{1 h} \underline{\sigma}_{p}, \underline{\Pi}_{1 h} \underline{\sigma}_{p}\right)}{\left\langle\Pi_{2 h} \chi_{p}, \Pi_{2 h} \chi_{p}\right\rangle_{0, \Omega_{h}}}\right] \leq \lambda_{p}+2 \max _{\substack{\left(\underline{\sigma}_{p}, \chi_{p}\right) \in \mathcal{M}_{p} \\
\left\|\chi_{p}\right\|_{0, \Omega}=1}}\left\{\left|\widetilde{\alpha}_{p, h}\right|\right\} \lambda_{p} \\
& +C h \max _{\substack{\left(\underline{\sigma}_{p}, \chi_{p}\right) \in \mathcal{M}_{p} \\
\left\|\chi_{p}\right\|_{0, \Omega}=1}}\left\{( 1 + 2 | \widetilde { \alpha } _ { p , h } | ) \left[h^{2}\left\|\underline{\widetilde{\sigma}}_{p}\right\|_{1, \widetilde{\Omega}}\left\|\widetilde{\chi}_{p}\right\|_{3, \widetilde{\Omega}}+\left(\left\|\widetilde{\widetilde{\sigma}}_{p}\right\|_{1, \widetilde{\Omega}}+\left\|\widetilde{\chi}_{p}\right\|_{3, \widetilde{\Omega}}\right)\right.\right. \\
& \left.\left.\times\left(h\left(\left\|\widetilde{\widetilde{\sigma}}_{p}\right\|_{1, \widetilde{\Omega}}+\left\|\widetilde{\chi}_{p}\right\|_{3, \widetilde{\Omega}}\right)+\left\|\underline{\widetilde{\sigma}}_{p}\right\|_{0, \widetilde{\Omega}}\right)+\|\widetilde{A}\|_{2, \infty, \widetilde{\Omega}}\left((1+h)\left\|\underline{\widetilde{\sigma}}_{p}\right\|_{1, \widetilde{\Omega}}+h\left\|\widetilde{\chi}_{p}\right\|_{3, \widetilde{\Omega}}\right)^{2}\right]\right\}
\end{aligned}
$$

where $\left|\widetilde{\alpha}_{p, h}\right| \leq\left(\left\|\chi_{p}\right\|_{0, \omega_{h}}^{2}+\left\|\widetilde{\chi}_{p}\right\|_{0, \epsilon_{h}}^{2}+\alpha_{p, h}\right) \longrightarrow 0$ as $h \longrightarrow 0$ by virtue of (4.16) and (4.17)

$$
\Longrightarrow \lim _{h \rightarrow 0} \max _{\substack{\left.\underline{\sigma}_{p}, \chi_{p}\right) \in \mathcal{M}_{p} \\\left\|\chi_{p}\right\|_{0, \Omega}=1}}\left|\widetilde{\alpha}_{p, h}\right|=0 \Longrightarrow \lim _{h \rightarrow 0} \lambda_{p, h} \leq \lambda_{p} .
$$

Now, we will show that $\lim _{h \rightarrow 0} \lambda_{p, h} \geq \lambda_{p}$. Let $\left(\mu_{m, h} ; u_{m, h}\right) \in \mathbb{R}^{+} \times W_{h}$ be the eigenpairs of $T_{h} \in \mathcal{L}\left(W_{h}\right)$ with $\widetilde{u}_{m, h} \in \widetilde{W}_{h} \subset H_{0}^{1}(\widetilde{\Omega}), \widetilde{u}_{m, h} \downarrow_{\Omega_{h}}=u_{m, h}$. For $U_{p, h}=\operatorname{Span}\left\{\left(u_{m, h}\right)_{m=1}^{p}\right\} \subset W_{h}$, let $\widetilde{U}_{p, h}=\operatorname{Span}\left\{\left(\widetilde{u}_{m, h}\right)_{m=1}^{p}\right\} \subset$ $\widetilde{W}_{h} \subset H_{0}^{1}(\widetilde{\Omega})$ be a p-dimensional subspace. Then, $v_{h} \in U_{p, h} \Longleftrightarrow \widetilde{v}_{h} \in \widetilde{U}_{p, h}$, and from Theorem 3.6, $\mu_{p, h}=\min _{v_{h} \in U_{p, h}} \frac{\left\langle T_{h} v_{h}, v_{h}\right\rangle_{0, \Omega_{h}}}{\left\langle v_{h}, v_{h}\right\rangle_{0, \Omega_{h}}}$. Under (A5), $T: \widetilde{v}_{h} \in L^{2}(\Omega) \mapsto T \widetilde{v}_{h} \in H^{3}(\Omega) \cap H_{0}^{2}(\Omega)$ with $\left\|T \widetilde{v}_{h}\right\|_{3, \Omega} \leq C\left\|\widetilde{v}_{h}\right\|_{0, \Omega}$ and $\widetilde{T \widetilde{v}_{h}} \in H^{3}(\widetilde{\Omega})$ such that $\left\|\widetilde{T \widetilde{v}_{h}}\right\|_{3, \widetilde{\Omega}} \leq C\left\|T \widetilde{v}_{h}\right\|_{3, \Omega}$ and $\widetilde{T \widetilde{v}_{h}} \downarrow_{\Omega_{h}} \in H^{3}\left(\Omega_{h}\right)$ will be denoted by $\widetilde{T \widetilde{v}_{h}}$ such that

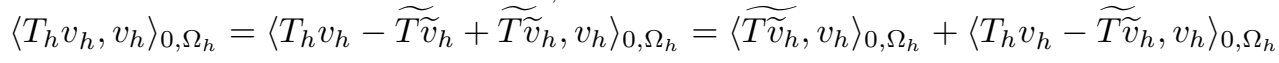

$$
\begin{aligned}
\Longrightarrow \mu_{p, h} & =\min _{v_{h} \in U_{p, h}, \widetilde{v}_{h} \in \widetilde{U}_{p, h}}\left[\frac{\left\langle\widetilde{T \widetilde{v}}_{h}, v_{h}\right\rangle_{0, \Omega_{h}}}{\left\langle v_{h}, v_{h}\right\rangle_{0, \Omega_{h}}}+\frac{\left\langle T_{h} v_{h}-{\widetilde{T \widetilde{v}_{h}}}_{h}, v_{h}\right\rangle_{0, \Omega_{h}}}{\left\langle v_{h}, v_{h}\right\rangle_{0, \Omega_{h}}}\right] \\
& \leq \min _{v_{h} \in U_{p, h}, \widetilde{v}_{h} \in \widetilde{U}_{p, h}}\left[\frac{\left\langle\widetilde{T \widetilde{v}}_{h}, v_{h}\right\rangle_{0, \Omega_{h}}}{\left\langle v_{h}, v_{h}\right\rangle_{0, \Omega_{h}}}\right]+\max _{v_{h} \in U_{p, h}, \widetilde{v}_{h} \in \widetilde{U}_{p, h}}\left[\frac{\left\langle T_{h} v_{h}-\widetilde{T \widetilde{v}_{h}}, v_{h}\right\rangle_{0, \Omega_{h}}}{\left\langle v_{h}, v_{h}\right\rangle_{0, \Omega_{h}}}\right]
\end{aligned}
$$

Since $\widetilde{v_{h}} \downarrow_{\omega_{h}=\Omega-\left(\Omega \cap \Omega_{h}\right)}=0,\left\langle\widetilde{T \widetilde{v}_{h}}, v_{h}\right\rangle_{0, \Omega_{h}}=\int_{\Omega_{h}}\left(\widetilde{T \widetilde{v}_{h}}\right) v_{h} \mathrm{~d} x=\left\langle T \widetilde{v}_{h}, \widetilde{v}_{h}\right\rangle_{0, \Omega}+\left\langle\widetilde{T \widetilde{v}_{h}}, v_{h}\right\rangle_{0, \epsilon_{h}}$

$$
\Longrightarrow \frac{\left\langle\widetilde{T \widetilde{v}_{h}}, v_{h}\right\rangle_{0, \Omega_{h}}}{\left\langle v_{h}, v_{h}\right\rangle_{0, \Omega_{h}}}=\frac{\left\langle T \widetilde{v}_{h}, \widetilde{v}_{h}\right\rangle_{0, \Omega}}{\left\langle v_{h}, v_{h}\right\rangle_{0, \Omega_{h}}}+\frac{\left\langle\widetilde{T \widetilde{v}_{h}}, v_{h}\right\rangle_{0, \epsilon_{h}}}{\left\langle v_{h}, v_{h}\right\rangle_{0, \Omega_{h}}} \forall v_{h} \in U_{p, h} \text { with } \widetilde{v}_{h} \in \widetilde{U}_{p, h},
$$

From (3.8), (4.5) and $\left\|\tilde{v}_{h}\right\|_{0, \Omega} \leq\left\|v_{h}\right\|_{0, \Omega_{h}}$,

$$
\left\|\widetilde{T \widetilde{v}_{h}}\right\|_{0, \epsilon_{h}} \leq C h^{3 / 2}\left\|\widetilde{T \widetilde{v}_{h}}\right\|_{1, \widetilde{\Omega}} \leq C h^{3 / 2}\left\|\widetilde{T \widetilde{v}_{h}}\right\|_{3, \widetilde{\Omega}} \leq C h^{3 / 2}\left\|T \widetilde{v}_{h}\right\|_{3, \Omega} \leq C h^{3 / 2}\left\|\widetilde{v}_{h}\right\|_{0, \Omega} \leq C h^{3 / 2}\left\|v_{h}\right\|_{0, \Omega_{h}}
$$




$$
\begin{aligned}
& \text { and }\left|\left\langle\widetilde{T \widetilde{v}_{h}}, v_{h}\right\rangle_{0, \epsilon_{h}}\right| \leq\left\|\widetilde{T \widetilde{v}_{h}}\right\|_{0, \epsilon_{h}}\left\|v_{h}\right\|_{0, \epsilon_{h}} \leq C h^{3 / 2}\left\|v_{h}\right\|_{0, \Omega_{h}}^{2}, \\
& \min _{v_{h} \in U_{p, h}, \widetilde{v}_{h} \in \widetilde{U}_{p, h}} \frac{\left\langle\widetilde{T \widetilde{v}_{h}}, v_{h}\right\rangle_{0, \Omega_{h}}}{\left\langle v_{h}, v_{h}\right\rangle_{0, \Omega_{h}}} \leq \min _{\widetilde{v}_{h} \in \widetilde{U}_{p, h} \subset L^{2}(\Omega)} \frac{\left\langle T \widetilde{v}_{h}, \widetilde{v}_{h}\right\rangle_{0, \Omega}}{\left\langle\widetilde{v}_{h}, \widetilde{v}_{h}\right\rangle_{0, \Omega}}+C h^{3 / 2} \leq \mu_{p}+C h^{3 / 2}
\end{aligned}
$$

(using $\left\|\tilde{v}_{h}\right\|_{0, \Omega} \leq\left\|v_{h}\right\|_{0, \Omega_{h}}$ and (4.33) in (4.32)).

Hence, from (4.31), $\mu_{p, h} \leq \mu_{p}+C h^{3 / 2}+\max _{v_{h} \in U_{p, h} \text { with } \widetilde{v}_{h} \in \widetilde{U}_{p, h}} \frac{\left|\left\langle T_{h} v_{h}-\widetilde{T \widetilde{v}_{h}}, v_{h}\right\rangle_{0, \Omega_{h}}\right|}{\left\langle v_{h}, v_{h}\right\rangle_{0, \Omega_{h}}}$.

But $\left|\left\langle T_{h} v_{h}-\widetilde{T \widetilde{v}_{h}}, v_{h}\right\rangle_{0, \Omega_{h}}\right| \leq\left\|T_{h} v_{h}-\widetilde{T \widetilde{v}_{h}}\right\|_{0, \Omega_{h}}\left\|v_{h}\right\|_{0, \Omega_{h}}$

and from Theorem 3.3, $\left\|T_{h} v_{h}-\widetilde{T \widetilde{v}_{h}}\right\|_{0, \Omega_{h}} \leq C h\left(\left\|\widetilde{T \widetilde{v}_{h}}\right\|_{3, \widetilde{\Omega}}+\left\|\widetilde{S \widetilde{v}_{h}}\right\|_{1, \widetilde{\Omega}}\right)$. Then, using (3.7)-(3.9),

$\left\|\widetilde{T \widetilde{v}_{h}}\right\|_{3, \widetilde{\Omega}} \leq C\left\|T \widetilde{v}_{h}\right\|_{3, \Omega} \leq C\left\|v_{h}\right\|_{0, \Omega_{h}}$ and $\left\|\widetilde{S \widetilde{v}_{h}}\right\|_{1, \widetilde{\Omega}} \leq C\left\|S \widetilde{v}_{h}\right\|_{1, \Omega} \leq C\left\|v_{h}\right\|_{0, \Omega_{h}}$

$\Longrightarrow\left|\left\langle T_{h} v_{h}-\widetilde{T \widetilde{v}_{h}}, v_{h}\right\rangle_{0, \Omega_{h}}\right| \leq C h\left\|v_{h}\right\|_{0, \Omega_{h}}^{2}$ and from (4.34), $\mu_{p, h} \leq \mu_{p}+C h^{3 / 2}+C h$

$\Longrightarrow \frac{1}{\lambda_{p, h}}-\frac{1}{\lambda_{p}} \leq C h(1+\sqrt{h}) \Longrightarrow \lim _{h \rightarrow 0} \lambda_{p, h} \geq \lambda_{p}$, which together with (4.30), gives the result.

Theorem 4.3. Under the assumption that Theorem 4.2 holds and $\lambda_{p}$ (resp. $\lambda_{p, h}$ ) is a simple eigenvalue of $\left(\mathbf{Q}^{\mathbf{E}}\right)\left(\right.$ resp. $\left.\left(\mathbf{Q}_{\mathbf{h}}^{\mathbf{E}}\right)\right), \exists C>0$, independent of ' $h$ ' and 'p', such that $\left.\forall h \in\right] 0, h_{0}\left[\right.$ with $\left.h_{0} \in\right] 0,1[$,

$$
\begin{aligned}
\left\|\widetilde{u}_{p}-u_{p, h}\right\|_{0, \Omega_{h}} \leq & C h\left[h^{2}\left\|\widetilde{u}_{p}\right\|_{1, \widetilde{\Omega}}^{2}+\left(\left\|\widetilde{u}_{p}\right\|_{3, \widetilde{\Omega}}+\left\|\widetilde{\Psi}_{p}\right\|_{1, \widetilde{\Omega}}\right) \times\left\{1+2 \frac{\lambda_{p}}{d_{p}}+(h+2)\left\|\widetilde{u}_{p}\right\|_{3, \widetilde{\Omega}}+h\left\|\widetilde{\Psi}_{p}\right\|_{1, \widetilde{\Omega}}\right\}\right] . \\
\left\|\widetilde{\Psi}_{p}-\Psi_{p, h}\right\|_{0, \Omega_{h} \leq} \leq & C h\left[\left(\left\|\widetilde{u}_{p}\right\|_{3, \widetilde{\Omega}}+\left\|\widetilde{\Psi}_{p}\right\|_{1, \widetilde{\Omega}}\right)\left(1+\frac{2 \lambda_{p} \sqrt{2}}{\sqrt{\lambda_{1} \bar{\alpha}_{0}}}\left\{1+2 \frac{\lambda_{p}}{d_{p}}+(h+2)\left\|\widetilde{u}_{p}\right\|_{3, \widetilde{\Omega}}+h\left\|\widetilde{\Psi}_{p}\right\|_{1, \widetilde{\Omega}}\right\}\right)\right. \\
& \left.+\frac{2 \sqrt{2} \lambda_{p}}{\sqrt{\lambda_{1} \bar{\alpha}_{0}}} h^{2}\left\|\widetilde{u}_{p}\right\|_{1, \widetilde{\Omega}}^{2}\right]+\frac{\sqrt{2}}{\sqrt{\lambda_{1} \bar{\alpha}_{0}}}\left|\lambda_{p}-\lambda_{p, h}\right| \text { with parameter } d_{p}>0 \text { defined in }(4.41) .
\end{aligned}
$$

Proof. Let $\underline{\underline{\Pi}}_{\mathbf{h}}: \mathcal{M}_{p} \longrightarrow \underline{\underline{\Pi}}_{\mathbf{h}} \mathcal{M}_{p} \subset \mathcal{M}_{h}$ be defined by (3.40) with $\underline{\sigma}_{p}=\Psi_{p}, \chi_{p}=u_{p}$ and $\widetilde{\chi}_{p}^{*}=\lambda_{p} \widetilde{u}_{p}$.

Then, choose $u_{p, h}$ such that $\left\langle\Pi_{2 h} u_{p}, u_{p, h}\right\rangle_{0, \Omega_{h}}>0$.

$$
\begin{aligned}
\left\|\widetilde{u}_{p}-u_{p, h}\right\|_{0, \Omega_{h}} \leq & \left\|\widetilde{u}_{p}-\Pi_{2 h} u_{p}\right\|_{0, \Omega_{h}}+\left\|\Pi_{2 h} u_{p}-\left\langle\Pi_{2 h} u_{p}, u_{p, h}\right\rangle_{0, \Omega_{h}} u_{p, h}\right\|_{0, \Omega_{h}} \\
& +\left\|\left\langle\Pi_{2 h} u_{p}, u_{p, h}\right\rangle_{0, \Omega_{h}} u_{p, h}-u_{p, h}\right\|_{0, \Omega_{h}} .
\end{aligned}
$$

We are to find estimates only for the second and third terms on the right hand side of (4.39), since (3.42) gives the estimate for the first term.

$\Pi_{2 h} u_{p} \in W_{h} \Longrightarrow \Pi_{2 h} u_{p}=\sum_{j=1}^{N_{h}}\left\langle\Pi_{2 h} u_{p}, u_{j, h}\right\rangle_{0, \Omega_{h}} u_{j, h}$ with $\left\langle u_{j, h}, u_{k, h}\right\rangle_{0, \Omega_{h}}=\delta_{j k}, 1 \leq j, k \leq N_{h}$.

From (3.21) and definition of $\underline{\Pi}_{\mathbf{h}}\left(\Psi_{p}, u_{p}\right)=\left(\underline{\Pi}_{1 h} \Psi_{p}, \Pi_{2 h} u_{p}\right)$ in (3.40), we have:

$$
\begin{aligned}
\lambda_{j, h}\left\langle\Pi_{2 h} u_{p}, u_{j, h}\right\rangle_{0, \Omega_{h}} & =-b_{h}^{\mathrm{NI}}\left(\Psi_{j, h}, \Pi_{2 h} u_{p}\right)=A_{h}^{\mathrm{NI}}\left(\underline{\Pi}_{1 h} \Psi_{p}, \Psi_{j, h}\right) \\
& =-b_{h}^{\mathrm{NI}}\left(\underline{\Pi}_{1 h} \Psi_{p}, u_{j, h}\right)=\lambda_{p}\left\langle\widetilde{u}_{p}, u_{j, h}\right\rangle_{0, \Omega_{h}} \text { with } \widetilde{\chi}_{p}^{*}=\lambda_{p} \widetilde{u}_{p} . \\
\Longrightarrow\left(\lambda_{j, h}-\lambda_{p}\right)\left\langle\Pi_{2 h} u_{p}, u_{j, h}\right\rangle_{0, \Omega_{h}} & =\lambda_{p}\left[\left\langle\widetilde{u}_{p}, u_{j, h}\right\rangle_{0, \Omega_{h}}-\left\langle\Pi_{2 h} u_{p}, u_{j, h}\right\rangle_{0, \Omega_{h}}\right] \\
\Longrightarrow\left\langle\Pi_{2 h} u_{p}, u_{j, h}\right\rangle_{0, \Omega_{h}} & =\frac{\lambda_{p}}{\left(\lambda_{j, h}-\lambda_{p}\right)}\left[\left\langle\widetilde{u}_{p}-\Pi_{2 h} u_{p}, u_{j, h}\right\rangle_{0, \Omega_{h}}\right](j \neq p) .
\end{aligned}
$$


Since we are considering the case of simple eigenvalues, set $2 d_{p}=\min \left\{\lambda_{p}-\lambda_{p-1}, \lambda_{p+1}-\lambda_{p}\right\}>0$.

From Theorem 4.2, $\lim _{h \rightarrow 0} \lambda_{j, h}=\lambda_{j}(j \neq p)$. Hence, $\left.\forall h \in\right] 0, h_{0}\left[\right.$ with some $\left.h_{0} \in\right] 0,1[$,

$$
\left|\lambda_{j, h}-\lambda_{p}\right| \geq d_{p} \Longrightarrow\left|\left\langle\Pi_{2 h} u_{p}, u_{j, h}\right\rangle_{0, \Omega_{h}}\right| \leq \frac{\lambda_{p}}{d_{p}}\left|\left\langle\widetilde{u}_{p}-\Pi_{2 h} u_{p}, u_{j, h}\right\rangle_{0, \Omega_{h}}\right| \forall j \neq p .
$$

But $\left(u_{j, h}\right)_{j=1}^{N_{h}}$ is orthonormal in $W_{h}$. Then,

$$
\begin{aligned}
& \left\|\Pi_{2 h} u_{p}-\left\langle\Pi_{2 h} u_{p}, u_{p, h}\right\rangle_{0, \Omega_{h}} u_{p, h}\right\|_{0, \Omega_{h}}=\left(\sum_{\substack{j=1 \\
j \neq p}}^{N_{h}}\left|\left\langle\Pi_{2 h} u_{p}, u_{j, h}\right\rangle_{0, \Omega_{h}}\right|^{2}\right)^{1 / 2} \\
& \left.\quad \leq\left(\frac{\lambda_{p}}{d_{p}}\right)\left(\sum_{\substack{j=1 \\
j \neq p}}^{N_{h}}\left|\left\langle\widetilde{u}_{p}-\Pi_{2 h} u_{p}, u_{j, h}\right\rangle_{0, \Omega_{h}}\right|^{2}\right)^{1 / 2} \leq\left(\frac{\lambda_{p}}{d_{p}}\right)\left\|\widetilde{u}_{p}-\Pi_{2 h} u_{p}\right\|_{0, \Omega_{h}} \forall h \in\right] 0, h_{0}\left[\text { with } h_{0}>0 .\right.
\end{aligned}
$$

Finally,

$$
\begin{aligned}
& \left\|\left\langle\Pi_{2 h} u_{p}, u_{p, h}\right\rangle_{0, \Omega_{h}} u_{p, h}-u_{p, h}\right\|_{0, \Omega_{h}}=\left|\left\|\left\langle\Pi_{2 h} u_{p}, u_{p, h}\right\rangle_{0, \Omega_{h}} u_{p, h}\right\|_{0, \Omega_{h}}-1\right| \\
& \leq\left|\left\|\left\langle\Pi_{2 h} u_{p}, u_{p, h}\right\rangle_{0, \Omega_{h}} u_{p, h}\right\|_{0, \Omega_{h}}-\left\|\Pi_{2 h} u_{p}\right\|_{0, \Omega_{h}}\right|+\left|\left\|\Pi_{2 h} u_{p}\right\|_{0, \Omega_{h}}-1\right| \\
& \leq\left\|\left\langle\Pi_{2 h} u_{p}, u_{p, h}\right\rangle_{0, \Omega_{h}} u_{p, h}-\Pi_{2 h} u_{p}\right\|_{0, \Omega_{h}}+\left|\left\|\Pi_{2 h} u_{p}\right\|_{0, \Omega_{h}}^{2}-1\right| \text {. } \\
& \text { i.e. }\left\|\left\langle\Pi_{2 h} u_{p}, u_{p, h}\right\rangle_{0, \Omega_{h}} u_{p, h}-u_{p, h}\right\|_{0, \Omega_{h}} \leq \frac{\lambda_{p}}{d_{p}}\left\|\widetilde{u}_{p}-\Pi_{2 h} u_{p}\right\|_{0, \Omega_{h}}+\left|\left\|\Pi_{2 h} u_{p}\right\|_{0, \Omega_{h}}^{2}-1\right|(\operatorname{using}(4.42)) \text {. } \\
& \leq\left(\left\|\Pi_{2 h} u_{p}\right\|_{0, \Omega_{h}}+\left\|\widetilde{u}_{p}\right\|_{0, \Omega_{h}}\right)\left|\left\|\Pi_{2 h} u_{p}\right\|_{0, \Omega_{h}}-\left\|\widetilde{u}_{p}\right\|_{0, \Omega_{h}}\right|+\left\|u_{p}\right\|_{0, \omega_{h}}^{2}+\left\|\widetilde{u}_{p}\right\|_{0, \epsilon_{h}}^{2} \\
& \leq\left(\left\|\Pi_{2 h} u_{p}-\widetilde{u}_{p}\right\|_{0, \Omega_{h}}+2\left\|\widetilde{u}_{p}\right\|_{0, \Omega_{h}}\right)\left\|\Pi_{2 h} u_{p}-\widetilde{u}_{p}\right\|_{0, \Omega_{h}}+C h^{3}\left\|\widetilde{u}_{p}\right\|_{1, \widetilde{\Omega}}^{2}
\end{aligned}
$$

From (4.43)-(4.44),

$$
\begin{aligned}
\|\left\langle\Pi_{2 h} u_{p}, u_{p, h}\right\rangle_{0, \Omega_{h}} u_{p, h} & -u_{p, h} \|_{0, \Omega_{h}} \\
& \leq\left\|\widetilde{u}_{p}-\Pi_{2 h} u_{p}\right\|_{0, \Omega_{h}}\left[\frac{\lambda_{p}}{d_{p}}+\left\|\widetilde{u}_{p}-\Pi_{2 h} u_{p}\right\|_{0, \Omega_{h}}+2\left\|\widetilde{u}_{p}\right\|_{0, \Omega_{h}}\right]+C h^{3}\left\|\widetilde{u}_{p}\right\|_{1, \widetilde{\Omega}}^{2}
\end{aligned}
$$

Finally, from (4.39), (4.42) and (4.45), we get

$$
\left\|\widetilde{u}_{p}-u_{p, h}\right\|_{0, \Omega_{h}} \leq\left\|\widetilde{u}_{p}-\Pi_{2 h} u_{p}\right\|_{0, \Omega_{h}}\left[1+2 \frac{\lambda_{p}}{d_{p}}+\left\|\widetilde{u}_{p}-\Pi_{2 h} u_{p}\right\|_{0, \Omega_{h}}+2\left\|\widetilde{u}_{p}\right\|_{1, \widetilde{\Omega}}\right]+C h^{3}\left\|\widetilde{u}_{p}\right\|_{1, \widetilde{\Omega}}^{2} .
$$

Then, using (3.42) with $\chi_{p}=u_{p}, \underline{\sigma}_{p}=\Psi_{p}$ in (4.46), we get the result (4.36).

Now, we proceed to prove (4.37). $\left\|\widetilde{\Psi}_{p}-\Psi_{p, h}\right\|_{0, \Omega_{h}} \leq\left\|\widetilde{\Psi}_{p}-\underline{\Pi}_{1 h} \Psi_{p}\right\|_{0, \Omega_{h}}+\left\|\underline{\Pi}_{1 h} \Psi_{p}-\Psi_{p, h}\right\|_{0, \Omega_{h}}$. 
From (3.28), $\left\|\underline{\Pi}_{1 h} \Psi_{p}-\Psi_{p, h}\right\|_{0, \Omega_{h}} \leq \frac{1}{\sqrt{\bar{\alpha}_{0}}}\left(A_{h}^{\mathrm{NI}}\left(\underline{\Pi}_{1 h} \Psi_{p}-\Psi_{p, h}, \underline{\Pi}_{1 h} \Psi_{p}-\Psi_{p, h}\right)\right)^{1 / 2}$

$$
=\left.\frac{1}{\sqrt{\bar{\alpha}_{0}}}\left\|\underline{\Pi}_{1 h} \Psi_{p}-\Psi_{p, h}\right\|\right|_{A_{h}^{\mathrm{NI}(\cdot, \cdot)}} \text {. }
$$

Setting $\widehat{\Psi}_{j, h}=\frac{\Psi_{j, h}}{\sqrt{\lambda_{j, h}}}$, we get $\left.\quad\left\|\underline{\Pi}_{1 h} \Psi_{p}-\Psi_{p, h}\right\|\right|_{A_{h}^{\mathrm{NI}}(\cdot, \cdot)} \leq\left\|\left|\underline{\Pi}_{1 h} \Psi_{p}-\left[\underline{\Pi}_{1 h} \Psi_{p}, \widehat{\Psi}_{p, h}\right]_{A_{h}^{\mathrm{NI}}(\cdot, \cdot)} \widehat{\Psi}_{p, h} \|\right|_{A_{h}^{\mathrm{NI}}(\cdot, \cdot)}\right.$

$$
+\left|\left[\underline{\Pi}_{1 h} \Psi_{p}, \widehat{\Psi}_{p, h}\right]_{A_{h}^{\mathrm{NI}}(\cdot, \cdot)}-\sqrt{\lambda_{p, h}}\right|, \text { since }\left[\widehat{\Psi}_{j, h}, \widehat{\Psi}_{p, h}\right]_{A_{h}^{\mathrm{NI}}(\cdot, \cdot)}=\delta_{j p} \text {. }
$$

But $\underline{\Pi}_{1 h} \Psi_{p} \in \mathcal{E}_{h} \Longrightarrow \underline{\Pi}_{1 h} \Psi_{p}=\sum_{j=1}^{N_{h}}\left[\underline{\Pi}_{1 h} \Psi_{p}, \widehat{\Psi}_{j, h}\right]_{A_{h}^{\mathrm{NI}}(\cdot, \cdot)} \widehat{\Psi}_{j, h}$ with

$$
\begin{aligned}
& {\left[\underline{\Pi}_{1 h} \Psi_{p}, \widehat{\Psi}_{j, h}\right]_{A_{h}^{\mathrm{NI}}(\cdot, \cdot)}=\frac{1}{\sqrt{\lambda_{j, h}}} A_{h}^{\mathrm{NI}}\left(\underline{\Pi}_{1 h} \Psi_{p}, \Psi_{j, h}\right)=\frac{-1}{\sqrt{\lambda_{j, h}}} b_{h}^{\mathrm{NI}}\left(\underline{\Pi}_{1 h} \Psi_{p}, u_{j, h}\right)=\frac{\lambda_{p}}{\sqrt{\lambda_{j, h}}}\left\langle\widetilde{u}_{p}, u_{j, h}\right\rangle_{0, \Omega_{h}}} \\
& \text { with } \lambda_{p} \widetilde{u}_{p}=\widetilde{\chi}_{p}^{*} \quad(\text { using }(3.21) \text { and (3.40)) } \\
& \Longrightarrow\left[\underline{\Pi}_{1 h} \Psi_{p}, \widehat{\Psi}_{j, h}\right]_{A_{h}^{\mathrm{NI}(\cdot, \cdot)}}=\frac{\lambda_{p}}{\sqrt{\lambda_{j, h}}}\left[\left\langle\widetilde{u}_{p}-u_{p, h}, u_{j, h}\right\rangle_{0, \Omega_{h}}\right] 1 \leq j \neq p \leq N_{h} .
\end{aligned}
$$

From Theorem 4.2, $\left.\lim _{h \rightarrow 0} \lambda_{j, h}=\lambda_{j}, 1 \leq j \leq N_{h} \Longrightarrow \exists h_{0} \in\right] 0,1[$ such that

$$
\lambda_{j, h} \geq \frac{\lambda_{1}}{2} \forall j=1,2, \cdots, N_{h} \Longrightarrow \sqrt{\lambda_{j, h}} \geq \sqrt{\frac{\lambda_{1}}{2}} .
$$

Hence, from (4.51) and (4.52), $\left.\forall j \neq p, \quad 1 \leq j \leq N_{h}, \forall h \in\right] 0, h_{0}\left[\right.$ with $\left.h_{0} \in\right] 0,1[$,

$$
\begin{gathered}
\left|\left[\underline{\Pi}_{1 h} \Psi_{p}, \widehat{\Psi}_{j, h}\right]_{A_{h}^{\mathrm{NI}}(\cdot, \cdot)}\right| \leq \lambda_{p} \sqrt{\frac{2}{\lambda_{1}}}\left|\left\langle\widetilde{u}_{p}-u_{p, h}, u_{j, h}\right\rangle_{0, \Omega_{h}}\right| \\
\text { and }\left\|\left|\underline{\Pi}_{1 h} \Psi_{p}-\left[\underline{\Pi}_{1 h} \Psi_{p}, \widehat{\Psi}_{p, h}\right]_{A_{h}^{\mathrm{NI}}(\cdot, \cdot)} \widehat{\Psi}_{p, h}\right|\right\|_{A_{h}^{\mathrm{NI}}(\cdot, \cdot)}=\left(\sum_{\substack{j=1 \\
j \neq p}}^{N_{h}}\left|\left\|\left[\underline{\Pi}_{1 h} \Psi_{p}, \widehat{\Psi}_{j, h}\right]_{A_{h}^{\mathrm{NI}}(\cdot, \cdot)} \widehat{\Psi}_{j, h} \mid\right\|_{A_{h}^{\mathrm{NI}}(\cdot, \cdot)}^{2}\right)^{1 / 2}\right. \\
\leq \frac{\sqrt{2}}{\sqrt{\lambda_{1}}}\left(\lambda_{p}\right)\left(\sum_{\substack{j=1 \\
j \neq p}}^{N_{h}}\left|\left\langle\widetilde{u}_{p}-u_{p, h}, u_{j, h}\right\rangle_{0, \Omega_{h}}\right|^{2}\right)^{1 / 2} \leq \frac{\sqrt{2}}{\sqrt{\lambda_{1}}}\left(\lambda_{p}\right)\left\|\widetilde{u}_{p}-u_{p, h}\right\|_{0, \Omega_{h}} \text { (by Bessel's inequality). }
\end{gathered}
$$

Putting $j=p$ in (4.50), $\left[\underline{\Pi}_{1 h} \Psi_{p}, \widehat{\Psi}_{p, h}\right]_{A_{h}^{\mathrm{NI}}(\cdot, \cdot)}=\frac{\lambda_{p}}{\sqrt{\lambda_{p, h}}}\left\langle\widetilde{u}_{p}, u_{p, h}>_{0, \Omega_{h}}\right.$. 
Therefore, $\left|\left[\underline{\Pi}_{1 h} \Psi_{p}, \widehat{\Psi}_{p, h}\right]_{A_{h}^{\mathrm{NI}(\cdot, \cdot)}}-\sqrt{\lambda_{p, h}}\right|=\frac{1}{\sqrt{\lambda_{p, h}}}\left|\lambda_{p}\left\langle\widetilde{u}_{p}, u_{p, h}\right\rangle_{0, \Omega_{h}}-\lambda_{p, h}\right|$

$$
\begin{aligned}
& =\frac{1}{\sqrt{\lambda_{p, h}}}\left|\lambda_{p}\left\langle\widetilde{u}_{p}-u_{p, h}, u_{p, h}\right\rangle_{0, \Omega_{h}}+\left(\lambda_{p}-\lambda_{p, h}\right)\right| \\
& \Longrightarrow \quad\left|\left[\underline{\Pi}_{1 h} \Psi_{p}, \widehat{\Psi}_{p, h}\right]_{A_{h}^{\mathrm{NI}(\cdot, \cdot)}}-\sqrt{\lambda_{p, h}}\right| \leq \frac{1}{\sqrt{\lambda_{p, h}}}\left[\lambda_{p}\left\|\widetilde{u}_{p}-u_{p, h}\right\|_{0, \Omega_{h}}+\left|\lambda_{p}-\lambda_{p, h}\right|\right] \\
& \leq \sqrt{\frac{2}{\lambda_{1}}}\left[\lambda_{p}\left\|\widetilde{u}_{p}-u_{p, h}\right\|_{0, \Omega_{h}}+\left|\lambda_{p}-\lambda_{p, h}\right|\right](\text { by }(4.52)) .
\end{aligned}
$$

Finally, from $(4.47),(4.48),(4.49),(4.53)$ and $(4.54)$, we get: $\forall h \in] 0, h_{0}\left[\right.$ with $\left.h_{0} \in\right] 0,1[$,

$$
\left\|\widetilde{\Psi}_{p}-\Psi_{p, h}\right\|_{0, \Omega_{h}} \leq\left\|\widetilde{\Psi}_{p}-\underline{\Pi}_{1 h} \Psi_{p}\right\|_{0, \Omega_{h}}+\frac{1}{\sqrt{\bar{\alpha}_{0}}} \sqrt{\frac{2}{\lambda_{1}}}\left[2 \lambda_{p}\left\|\widetilde{u}_{p}-u_{p, h}\right\|_{0, \Omega_{h}}+\left|\lambda_{p}-\lambda_{p, h}\right|\right] .
$$

Using the estimates (3.42) and (4.36) for $\left\|\widetilde{\Psi}_{p}-\underline{\Pi}_{1 h} \Psi_{p}\right\|_{0, \Omega_{h}}$ and $\left\|\widetilde{u}_{p}-u_{p, h}\right\|_{0, \Omega_{h}}$ respectively in (4.55), we get the result (4.37).

Now, we proceed to find the estimate for the term $\left|\lambda_{p}-\lambda_{p, h}\right|$ occurring in (4.37).

Theorem 4.4. Under the assumptions that Theorems 4.2 and 4.3 hold and $\lambda_{p}$ (resp. $\lambda_{p, h}$ ) is a simple eigenvalue of $\left(\mathbf{Q}^{\mathbf{E}}\right)$ (resp. $\left.\left.\left(\mathbf{Q}_{\mathbf{h}}^{\mathbf{E}}\right)\right), 1 \leq p \leq N_{h}=\operatorname{dim} W_{h}, \forall h \in\right] 0, h_{0}\left[\right.$ with $\left.h_{0} \in\right] 0,1[, \exists C>0$, independent of ' $h$ ' and ' $p$ ' such that

$$
\begin{aligned}
& \left|\lambda_{p}-\lambda_{p, h}\right| \leq C h^{2}\left\{( \| \widetilde { u } _ { p } \| _ { 3 , \widetilde { \Omega } } + \| \widetilde { \Psi } _ { p } \| _ { 1 , \widetilde { \Omega } } ) \left[\lambda _ { p } \left(h^{2}\left\|\widetilde{u}_{p}\right\|_{1, \widetilde{\Omega}}^{2}+\left(\left\|\widetilde{u}_{p}\right\|_{3, \widetilde{\Omega}}+\left\|\widetilde{\Psi}_{p}\right\|_{1, \widetilde{\Omega}}\right)\left(1+\frac{2 \lambda_{p}}{d_{p}}+(h+2)\left\|\widetilde{u}_{p}\right\|_{3, \widetilde{\Omega}}\right.\right.\right.\right. \\
& \left.\left.\left.\left.\quad+h\left\|\widetilde{\Psi}_{p}\right\|_{1, \widetilde{\Omega}}\right)\right)+(h+2)\left\|\widetilde{u}_{p}\right\|_{3, \widetilde{\Omega}}+\left\|\widetilde{\Psi}_{p}\right\|_{1, \widetilde{\Omega}}\right]+\left(\lambda_{p} h\left\|\widetilde{u}_{p}\right\|_{3, \widetilde{\Omega}}+(h+2)\left\|\widetilde{\Psi}_{p}\right\|_{1, \widetilde{\Omega}}\right)\left\|\widetilde{u}_{p}\right\|_{3, \widetilde{\Omega}}\right\}=O\left(h^{2}\right)
\end{aligned}
$$

Proof. Let $\underline{\Pi}_{\mathbf{h}}: \mathcal{M}_{p} \longrightarrow \underline{\Pi}_{\mathbf{h}} \mathcal{M}_{p} \subset \mathcal{M}_{h}$ with $\underline{\Pi}_{\mathbf{h}}\left(\Psi_{p}, u_{p}\right)=\left(\underline{\Pi}_{1 h} \Psi_{p}, \Pi_{2 h} u_{p}\right)$ be defined by (3.40) such that the estimates $(3.42)$ hold, $\left(\lambda_{p} ;\left(\Psi_{p}, u_{p}\right)\right) \in \mathbb{R}^{+} \times(\mathbf{V} \times W)\left(\operatorname{resp} .\left(\lambda_{p, h} ;\left(\Psi_{p, h}, u_{p, h}\right)\right) \in \mathbb{R}^{+} \times\left(\mathbf{V}_{h} \times W_{h}\right)\right)$ with $u_{p} \in H^{3}(\Omega) \cap H_{0}^{2}(\Omega),\left\|u_{p}\right\|_{0, \Omega}=1$ being an eigenpair of $\left(\mathbf{Q}^{\mathbf{E}}\right)\left(\operatorname{resp} .\left(\mathbf{Q}_{\mathbf{h}}^{\mathbf{E}}\right)\right)$. We have from (3.40) and (3.21), $b_{h}^{\mathrm{NI}}\left(\Psi_{p, h}, \Pi_{2 h} u_{p}\right)=-\lambda_{p}\left\langle\widetilde{u}_{p}, u_{p, h}\right\rangle_{0, \Omega_{h}}$, and from $(3.21)$,

$$
\begin{aligned}
\lambda_{p}-\lambda_{p, h}= & \lambda_{p}-\frac{\lambda_{p}\left\langle\widetilde{u}_{p}, u_{p, h}\right\rangle_{0, \Omega_{h}}}{\left\langle u_{p, h}, \Pi_{2 h} u_{p}\right\rangle_{0, \Omega_{h}}}=\frac{\lambda_{p}}{\left\langle u_{p, h}, \Pi_{2 h} u_{p}\right\rangle_{0, \Omega_{h}}}\left[-\left\langle\widetilde{u}_{p}-\Pi_{2 h} u_{p}, u_{p, h}\right\rangle_{0, \Omega_{h}}\right] \\
& \text { with } \tilde{u}_{p} \in H^{3}(\widetilde{\Omega}), \tilde{u}_{p} \downarrow_{\Omega}=u_{p} \in H^{3}(\Omega) \cap H_{0}^{2}(\Omega) .
\end{aligned}
$$

$$
\text { But }-\left\langle\widetilde{u}_{p}-\Pi_{2 h} u_{p}, u_{p, h}>_{0, \Omega_{h}}=\left\langle\widetilde{u}_{p}-\Pi_{2 h} u_{p}, \widetilde{u}_{p}-u_{p, h}\right\rangle_{0, \Omega_{h}}-\left\langle\widetilde{u}_{p}-\Pi_{2 h} u_{p}, \widetilde{u}_{p}\right\rangle_{0, \Omega_{h}}\right.
$$

Using (3.40) with $\widetilde{\chi}_{p}^{*}=\widetilde{u}_{p}$ and (2.20), we have:

$$
\begin{aligned}
-\left\langle\widetilde{u}_{p}\right. & \left.-\Pi_{2 h} u_{p}, \widetilde{u}_{p}\right\rangle_{0, \Omega_{h}}=-\left\langle u_{p}, u_{p}\right\rangle_{0, \Omega}-\left\langle\widetilde{u}_{p}, \widetilde{u}_{p}\right\rangle_{0, \epsilon_{h}}+\left\langle u_{p}, u_{p}\right\rangle_{0, \omega_{h}}-\lambda_{p}^{-1} b_{h}^{\mathrm{NI}}\left(\underline{\Pi}_{1 h} \Psi_{p}, \Pi_{2 h} u_{p}\right) \\
& =\lambda_{p}^{-1}\left[\widetilde{b}_{h}\left(\widetilde{\Psi}_{p}, \widetilde{u}_{p}\right)-b_{h}^{\mathrm{NI}}\left(\underline{\Pi}_{1 h} \Psi_{p}, \Pi_{2 h} u_{p}\right)\right]+\left[\left\|u_{p}\right\|_{0, \omega_{h}}^{2}-\left\|\widetilde{u}_{p}\right\|_{0, \epsilon_{h}}^{2}\right]+\lambda_{p}^{-1}\left[b\left(\Psi_{p}, u_{p}\right)-{\widetilde{b_{h}}}_{(}\left(\widetilde{\Psi}_{p}, \widetilde{u}_{p}\right)\right] .
\end{aligned}
$$


From (4.57)-(4.59),

$$
\begin{aligned}
\lambda_{p}-\lambda_{p, h}=\frac{1}{\left\langle u_{p, h}, \Pi_{2 h} u_{p}\right\rangle_{0, \Omega_{h}}}\{ & \lambda_{p}\left\langle\widetilde{u}_{p}-\Pi_{2 h} u_{p}, \widetilde{u}_{p}-u_{p, h}\right\rangle_{0, \Omega_{h}}+\lambda_{p}\left(\left\|u_{p}\right\|_{0, \omega_{h}}^{2}-\left\|\widetilde{u}_{p}\right\|_{0, \epsilon_{h}}^{2}\right) \\
& \left.+\left[b\left(\Psi_{p}, u_{p}\right)-\widetilde{b}_{h}\left(\widetilde{\Psi}_{p}, \widetilde{u}_{p}\right)\right]+\left[\widetilde{b}_{h}\left(\widetilde{\Psi}_{p}, \widetilde{u}_{p}\right)-b_{h}^{\mathrm{NI}}\left(\underline{\Pi}_{1 h} \Psi_{p}, \Pi_{2 h} u_{p}\right)\right]\right\} .
\end{aligned}
$$

$$
\begin{aligned}
\text { But } \widetilde{b_{h}}\left(\widetilde{\Psi}_{p}, \widetilde{u}_{p}\right)-b_{h}^{\mathrm{NI}}\left(\underline{\Pi}_{1 h} \Psi_{p}, \Pi_{2 h} u_{p}\right)= & \left.\widetilde{b_{h}}\left(\widetilde{\Psi}_{p}-\underline{\Pi}_{1 h} \Psi_{p}, \widetilde{u}_{p}-\chi_{h}\right)+\left[\widetilde{b_{h}}\left(\widetilde{\Psi}_{p}, \chi_{h}\right)-{\widetilde{b_{h}}}_{\left(\Pi_{1 h}\right.} \Psi_{p}, \chi_{h}\right)\right] \\
& +\left[\widetilde{b_{h}}\left(\underline{\Pi}_{1 h} \Psi_{p}, \widetilde{u}_{p}\right)-b_{h}^{\mathrm{NI}}\left(\underline{\Pi}_{1 h} \Psi_{p}, \Pi_{2 h} u_{p}\right)\right] \forall \chi_{h} \in W_{h} .
\end{aligned}
$$

Rewriting one by one the expressions in square brackets in (4.61) using (2.20) and (3.21):

$$
\begin{aligned}
{\left[\widetilde{b_{h}}\left(\widetilde{\Psi}_{p}, \chi_{h}\right)-\widetilde{b_{h}}\left(\underline{\Pi}_{1 h} \Psi_{p}, \chi_{h}\right)\right]=} & \left\{\widetilde{b_{h}}\left(\widetilde{\Psi}_{p}, \chi_{h}-\widetilde{u}_{p}\right)+\widetilde{b_{h}}\left(\widetilde{\Psi}_{p}, \widetilde{u}_{p}\right)-b_{h}^{\mathrm{NI}}\left(\underline{\Pi}_{1 h} \Psi_{p}, \chi_{h}\right)\right. \\
& \left.+\left[b_{h}^{\mathrm{NI}}\left(\underline{\Pi}_{1 h} \Psi_{p}, \chi_{h}\right)-\widetilde{b_{h}}\left(\underline{\Pi}_{1 h} \Psi_{p}, \chi_{h}\right)\right]\right\} \\
= & \left\{\widetilde{b_{h}}\left(\widetilde{\Psi}_{p}, \chi_{h}-\widetilde{u}_{p}\right)+\lambda_{p}\left(\widetilde{u}_{p}, \chi_{h}-\widetilde{u}_{p}\right\rangle_{0, \Omega_{h}}+\left[\widetilde{b_{h}}\left(\widetilde{\Psi}_{p}, \widetilde{u}_{p}\right)-b\left(\Psi_{p}, u_{p}\right)\right]\right. \\
& \left.+\lambda_{p}\left[\left\|\widetilde{u}_{p}\right\|_{0, \epsilon_{h}}^{2}-\left\|u_{p}\right\|_{0, \omega_{h}}^{2}\right]+\left[b_{h}^{\mathrm{NI}}\left(\underline{\Pi}_{1 h} \Psi_{p}, \chi_{h}\right)-{\widetilde{b_{h}}}\left(\underline{\Pi}_{1 h} \Psi_{p}, \chi_{h}\right)\right]\right\} .
\end{aligned}
$$

Let $\underline{\Pi}_{1 h} \Psi_{p} \in \widetilde{\mathbf{V}}_{h} \subset \widetilde{\mathbf{V}}$ be an extension to $\widetilde{\Omega}$ of $\underline{\Pi}_{1 h} \Psi_{p} \in \mathbf{V}_{h}$ defined in (3.7)-(3.9) with the help of Corollary 3.1. Then, using (3.21) and (2.20), we have

$$
\begin{aligned}
& \widetilde{b_{h}}\left(\underline{\Pi}_{1 h} \Psi_{p}, \widetilde{u}_{p}\right)-b_{h}^{\mathrm{NI}}\left(\underline{\Pi}_{1 h} \Psi_{p}, \Pi_{2 h} u_{p}\right)=\tilde{A}_{h}\left(\widetilde{\Psi}_{p}-\underline{\Pi}_{1 h} \Psi_{p}, \widetilde{\Psi}_{p}-\underline{\Pi}_{1 h} \Psi_{p}\right)+\left[-\widetilde{A_{h}}\left(\widetilde{\Psi}_{p}, \widetilde{\Psi}_{p}\right)+\widetilde{A_{h}}\left(\underline{\Pi}_{1 h} \Psi_{p}, \widetilde{\Psi}_{p}\right)\right] \\
& +\left[\tilde{A}_{h}\left(\widetilde{\Psi}_{p}, \underline{\Pi}_{1 h} \Psi_{p}\right)-A\left(\Psi_{p}, \widetilde{\Pi}_{1 h} \Psi_{p}\right)\right]+\left[A_{h}^{\mathrm{NI}}\left(\underline{\Pi}_{1 h} \Psi_{p}, \underline{\Pi}_{1 h} \Psi_{p}\right)-\widetilde{A_{h}}\left(\underline{\Pi}_{1 h} \Psi_{p}, \underline{\Pi}_{1 h} \Psi_{p}\right)\right] \\
& \left.+\left[{\widetilde{b_{h}}}_{\left(\underline{\Pi}_{1 h}\right.} \Psi_{p}, \widetilde{u}_{p}\right)-b\left(\widetilde{\Pi}_{1 h} \Psi_{p}, u_{p}\right)\right] \text { in which } \\
& \left.\left[-\tilde{A}_{h}\left(\widetilde{\Psi}_{p}, \widetilde{\Psi}_{p}\right)+\tilde{A}_{h}\left(\underline{\Pi}_{1 h} \Psi_{p}, \widetilde{\Psi}_{p}\right)\right]=\widetilde{b_{h}}\left(\widetilde{\Psi}_{p}-\underline{\Pi}_{1 h} \Psi_{p}, \widetilde{u}_{p}-\chi_{h}\right)+\left[\widetilde{b_{h}}\left(\widetilde{\Psi}_{p}, \chi_{h}\right)-{\widetilde{b_{h}}}_{\left(\Pi_{1 h}\right.} \Psi_{p}, \chi_{h}\right)\right] \\
& +\left[b\left(\Psi_{p}, u_{p}\right)-\widetilde{b_{h}}\left(\widetilde{\Psi}_{p}, \widetilde{u}_{p}\right)\right]+\left[\widetilde{b_{h}}\left(\underline{\Pi}_{1 h} \Psi_{p}, \widetilde{u}_{p}\right)-b\left(\widetilde{\Pi}_{1 h} \Psi_{p}, u_{p}\right)\right] \\
& +\left[A\left(\Psi_{p}, \Psi_{p}\right)-\tilde{A}_{h}\left(\widetilde{\Psi}_{p}, \widetilde{\Psi}_{p}\right)\right]+\left[\tilde{A}_{h}\left(\underline{\Pi}_{1 h} \Psi_{p}, \widetilde{\Psi}_{p}\right)-A\left(\widetilde{\Pi}_{1 h} \Psi_{p}, \Psi_{p}\right)\right] \quad \forall \chi_{h} \in W_{h}
\end{aligned}
$$


with the term $\left[\widetilde{b_{h}}\left(\widetilde{\Psi}_{p}, \chi_{h}\right)-\widetilde{b_{h}}\left(\underline{\Pi}_{1 h} \Psi_{p}, \chi_{h}\right)\right]$ in (4.64) being the same one considered earlier in (4.62). Hence, substituting (4.62)-(4.64) in (4.61), using it in (4.60) and applying triangular inequality, we have

$$
\begin{aligned}
& \left|\lambda_{p}-\lambda_{p, h}\right| \leq \frac{1}{\left|\left\langle u_{p, h}, \Pi_{2 h} u_{p}\right\rangle_{0, \Omega_{h}}\right|}\left\{\lambda_{p}\left|\left\langle\widetilde{u}_{p}-\Pi_{2 h} u_{p}, \widetilde{u}_{p}-u_{p, h}\right\rangle_{0, \Omega_{h}}\right|+\lambda_{p}\left(\left\|u_{p}\right\|_{0, \omega_{h}}^{2}+\left\|\widetilde{u}_{p}\right\|_{0, \epsilon_{h}}^{2}\right)\right. \\
& +2\left(\left|\tilde{A}_{h}\left(\widetilde{\Psi}_{p}, \underline{\Pi}_{1 h} \Psi_{p}\right)-A\left(\Psi_{p}, \widetilde{\Pi}_{1 h} \Psi_{p}\right)\right|+\left|\widetilde{b_{h}}\left(\widetilde{\Psi}_{p}-\underline{\Pi}_{1 h} \Psi_{p}, \widetilde{u}_{p}-\chi_{h}\right)\right|+\left|\widetilde{b}_{h}\left(\underline{\Pi}_{1 h} \Psi_{p}, \widetilde{u}_{p}\right)-b\left(\widetilde{\Pi}_{1 h} \Psi_{p}, u_{p}\right)\right|\right. \\
& \left.+\left|b_{h}{ }^{\mathrm{NI}}\left(\underline{\Pi}_{1 h} \Psi_{p}, \chi_{h}\right)-\widetilde{b_{h}}\left(\underline{\Pi}_{1 h} \Psi_{p}, \chi_{h}\right)\right|+\left|\widetilde{b_{h}}\left(\widetilde{\Psi}_{p}, \chi_{h}-\widetilde{u}_{p}\right)\right|+\lambda_{p}\left|\left\langle\widetilde{u}_{p}, \chi_{h}-\widetilde{u}_{p}\right\rangle_{0, \Omega_{h}}\right|\right) \\
& +\left|A_{h}^{\mathrm{NI}}\left(\underline{\Pi}_{1 h} \Psi_{p}, \underline{\Pi}_{1 h} \Psi_{p}\right)-\tilde{A}_{h}\left(\underline{\Pi}_{1 h} \Psi_{p}, \underline{\Pi}_{1 h} \Psi_{p}\right)\right|+\left|A\left(\Psi_{p}, \Psi_{p}\right)-\tilde{A}_{h}\left(\widetilde{\Psi}_{p}, \widetilde{\Psi}_{p}\right)\right| \\
& \left.+\left|\widetilde{A}\left(\widetilde{\Psi}_{p}-\underline{\Pi}_{1 h} \Psi_{p}, \widetilde{\Psi}_{p}-\underline{\Pi}_{1 h} \Psi_{p}\right)\right|\right\} \forall \chi_{h} \in W_{h} \text { (see [8] for details). }
\end{aligned}
$$

First of all, we will prove that $\left\langle u_{p, h}, \Pi_{2 h} u_{p}\right\rangle_{0, \Omega_{h}} \rightarrow 1$ as $h \rightarrow 0$. In fact,

$$
\left|\left\langle u_{p}, u_{p}\right\rangle_{0, \Omega}-\left\langle u_{p, h}, \Pi_{2 h} u_{p}\right\rangle_{0, \Omega_{h}}\right| \leq\left|\int_{\Omega \cap \Omega_{h}} u_{p}^{2}-u_{p, h} \Pi_{2 h} u_{p} \mathrm{~d} x\right|+\int_{\omega_{h}} u_{p}^{2} \mathrm{~d} x+\left|\int_{\epsilon_{h}}\left(u_{p, h}\right)\left(\Pi_{2 h} u_{p}\right) \mathrm{d} x\right| .
$$

But using (4.5), $\int_{\omega_{h}} u_{p}^{2} \mathrm{~d} x=\left\|u_{p}\right\|_{0, \omega_{h}}^{2} \leq C h^{3}\left\|\widetilde{u}_{p}\right\|_{1, \widetilde{\Omega}}^{2} \rightarrow 0$ as $h \rightarrow 0 \Longrightarrow \lim _{h \rightarrow 0} \int_{\omega_{h}} u_{p}^{2} \mathrm{~d} x=0$.

$\left|\int_{\epsilon_{h}}\left(u_{p, h}\right)\left(\Pi_{2 h} u_{p}\right) \mathrm{d} x\right| \leq\left\|u_{p, h}\right\|_{0, \epsilon_{h}}\left\|\Pi_{2 h} u_{p}\right\|_{0, \epsilon_{h}} \rightarrow 0$ as $h \rightarrow 0$. (using (4.5) and (4.36))

$$
\text { and } \begin{aligned}
\left|\int_{\Omega \cap \Omega_{h}}\left(u_{p}^{2}-u_{p, h} \Pi_{2 h} u_{p}\right) \mathrm{d} x\right| & \leq\left|\int_{\Omega \cap \Omega_{h}}\left(u_{p}^{2}-u_{p} . u_{p, h}\right) \mathrm{d} x\right|+\left|\int_{\Omega \cap \Omega_{h}} u_{p} u_{p, h}-u_{p, h} \Pi_{2 h} u_{p} \mathrm{~d} x\right| \\
& \leq\left\|u_{p}\right\|_{0, \Omega \cap \Omega_{h}}\left\|u_{p}-u_{p, h}\right\|_{0, \Omega \cap \Omega_{h}}+\left\|u_{p, h}\right\|_{0, \Omega \cap \Omega_{h}}\left\|u_{p}-\Pi_{2 h} u_{p}\right\|_{0, \Omega \cap \Omega_{h}} \\
& \leq\left[\left\|u_{p}\right\|_{0, \Omega}\left\|\widetilde{u}_{p}-u_{p, h}\right\|_{0, \Omega_{h}}+\left\|u_{p, h}\right\|_{0, \Omega_{h}}\left\|\widetilde{u}_{p}-\Pi_{2 h} u_{p}\right\|_{0, \Omega_{h}}\right] \rightarrow 0 \text { as } h \rightarrow 0,
\end{aligned}
$$

(from (4.36) and (3.42)). Hence, from (4.66),

$$
\begin{aligned}
\lim _{h \rightarrow 0}\left|\left\langle u_{p}, u_{p}\right\rangle_{0, \Omega}-\left\langle u_{p, h}, \Pi_{2 h} u_{p}\right\rangle_{0, \Omega_{h}}\right|=0 & \Longrightarrow \lim _{h \rightarrow 0}\left\langle u_{p, h}, \Pi_{2 h} u_{p}\right\rangle_{0, \Omega_{h}}=1 \\
& \left.\Longrightarrow \exists h_{0} \in\right] 0,1[\text { such that } \forall h \in] 0, h_{0}\left[\left|\left\langle u_{p, h}, \Pi_{2 h} u_{p}\right\rangle_{0, \Omega_{h}}\right|>1 / 2\right. \\
& \Longrightarrow \frac{1}{\left|\left\langle u_{p, h}, \Pi_{2 h} u_{p}\right\rangle_{0, \Omega_{h}}\right|}<2 .
\end{aligned}
$$


Using (3.42) and (4.36), we have

- $\lambda_{p}\left|\left\langle\widetilde{u}_{p}-\Pi_{2 h} u_{p}, \widetilde{u}_{p}-u_{p, h}\right\rangle_{0, \Omega_{h}}\right| \leq \lambda_{p}\left\|\widetilde{u}_{p}-\Pi_{2 h} u_{p}\right\|_{0, \Omega_{h}}\left\|\widetilde{u}_{p}-u_{p, h}\right\|_{0, \Omega_{h}}$

$$
\begin{aligned}
\leq C \lambda_{p} h^{2}\{ & \left(\left\|\widetilde{u}_{p}\right\|_{3, \widetilde{\Omega}}+\left\|\widetilde{\Psi}_{p}\right\|_{1, \widetilde{\Omega}}\right)\left[h^{2}\left\|\widetilde{u}_{p}\right\|_{1, \widetilde{\Omega}}^{2}+\left(\left\|\widetilde{u}_{p}\right\|_{3, \widetilde{\Omega}}\right.\right. \\
& \left.\left.\left.+\left\|\widetilde{\Psi}_{p}\right\|_{1, \widetilde{\Omega}}\right)\left(1+2 \frac{\lambda_{p}}{d_{p}}+(2+h)\left\|\widetilde{u}_{p}\right\|_{3, \widetilde{\Omega}}+h\left\|\widetilde{\Psi}_{p}\right\|_{1, \widetilde{\Omega}}\right)\right]\right\} ;
\end{aligned}
$$

$\bullet \lambda_{p}\left(\left\|\widetilde{u}_{p}\right\|_{0, \epsilon_{h}}^{2}+\left\|u_{p}\right\|_{0, \omega_{h}}^{2}\right) \leq C \lambda_{p} h^{3}\left\|\widetilde{u}_{p}\right\|_{1, \widetilde{\Omega}}^{2}(\operatorname{using}(4.5))$;

$$
\text { - }\left|A\left(\Psi_{p}, \widetilde{\underline{\Pi}_{1 h} \Psi_{p}}\right)-\tilde{A}_{h}\left(\widetilde{\Psi}_{p}, \underline{\Pi}_{1 h} \Psi_{p}\right)\right| \leq C h^{3}\left\|\widetilde{u}_{p}\right\|_{3, \widetilde{\Omega}}\left\|\underline{\Pi}_{1 h} \Psi_{p}\right\|_{1, \Omega_{h}} \quad \text { (see (II) in Prop. 4.2). }
$$

Estimate for $\left\|\underline{\Pi}_{1 h} \Psi_{p}\right\|_{1, \Omega_{h}}$ is given now: $\left\|\underline{\Pi}_{1 h} \Psi_{p}\right\|_{1, \Omega_{h}} \leq\left\|\underline{\Pi}_{1 h} \Psi_{p}-\widetilde{\Psi}_{p}\right\|_{1, \Omega_{h}}+\left\|\widetilde{\Psi}_{p}\right\|_{1, \Omega_{h}}$

But from (4.11), for $\widetilde{\Psi}_{p} \in \widetilde{\mathbf{V}}, \exists\left(\Theta_{h}\right)_{p} \in \mathbf{V}_{h}$ such that $\left\|\widetilde{\Psi}_{p}-\left(\Theta_{h}\right)_{p}\right\|_{r, \Omega_{h}} \leq C h^{1-r}\left\|\widetilde{\Psi}_{p}\right\|_{1, \widetilde{\Omega}}(r=0,1)$ with $\left\|\widetilde{\Psi}_{p}-\underline{\Pi}_{1 h} \Psi_{p}\right\|_{1, \Omega_{h}} \leq\left\|\underline{\Pi}_{1 h} \Psi_{p}-\left(\Theta_{h}\right)_{p}\right\|_{1, \Omega_{h}}+\left\|\widetilde{\Psi}_{p}-\left(\Theta_{h}\right)_{p}\right\|_{1, \Omega_{h}} \leq C / h\left\|\underline{\Pi}_{1 h} \Psi_{p}-\left(\Theta_{h}\right)_{p}\right\|_{0, \Omega_{h}}+C\left\|\widetilde{\Psi}_{p}\right\|_{1, \widetilde{\Omega}}$

$$
\begin{aligned}
& \text { and }\left\|\underline{\Pi}_{1 h} \Psi_{p}-\left(\Theta_{h}\right)_{p}\right\|_{0, \Omega_{h}} \leq\left\|\underline{\Pi}_{1 h} \Psi_{p}-\widetilde{\Psi}_{p}\right\|_{0, \Omega_{h}}+\left\|\widetilde{\Psi}_{p}-\left(\Theta_{h}\right)_{p}\right\|_{0, \Omega_{h}} \text {. Then, using (3.40), } \\
& \left\|\underline{\Pi}_{1 h} \Psi_{p}-\left(\Theta_{h}\right)_{p}\right\|_{0, \Omega_{h}} \leq C h\left(\left\|\widetilde{u}_{p}\right\|_{3, \widetilde{\Omega}}+\left\|\widetilde{\Psi}_{p}\right\|_{1, \widetilde{\Omega}}\right), \\
& \Longrightarrow\left\|\underline{\Pi}_{1 h} \Psi_{p}-\widetilde{\Psi}_{p}\right\|_{1, \Omega_{h}} \leq C\left(\left\|\widetilde{u}_{p}\right\|_{3, \widetilde{\Omega}}+\left\|\widetilde{\Psi}_{p}\right\|_{1, \widetilde{\Omega}}\right),\left\|\underline{\Pi}_{1 h} \Psi_{p}\right\|_{1, \Omega_{h}} \leq C\left(\left\|\widetilde{u}_{p}\right\|_{3, \widetilde{\Omega}}+\left\|\widetilde{\Psi}_{p}\right\|_{1, \widetilde{\Omega}}\right) .
\end{aligned}
$$

Finally from (4.70) and (4.71), we have

$$
\text { - }\left|A\left(\Psi_{p}, \widetilde{\Pi_{1 h} \Psi_{p}}\right)-\tilde{A}_{h}\left(\widetilde{\Psi}_{p}, \underline{\Pi}_{1 h} \Psi_{p}\right)\right| \leq C h^{3}\left\|\widetilde{u}_{p}\right\|_{3, \tilde{\Omega}}\left(\left\|\widetilde{u}_{p}\right\|_{3, \tilde{\Omega}}+\left\|\Psi_{p}\right\|_{1, \tilde{\Omega}}\right) .
$$

Using the continuity of $\tilde{A}_{h}(\cdot, \cdot), \widetilde{b}_{h}(\cdot, \cdot)$, Proposition 4.2, estimates (3.42), (4.5), (4.9), (4.12), (4.37) and (4.71), we have: For $\chi_{h}=\mathcal{P}_{h} \widetilde{u}_{p} \in W_{h}$

- $\left|\widetilde{b}_{h}\left(\widetilde{\Psi}_{p}-\underline{\Pi}_{1 h} \Psi_{p}, \widetilde{u}_{p}-\mathcal{P}_{h} \widetilde{u}_{p}\right)\right| \leq C h^{2}\left\|\widetilde{u}_{p}\right\|_{3, \widetilde{\Omega}}\left(\left\|\widetilde{u}_{p}\right\|_{3, \widetilde{\Omega}}+\left\|\widetilde{\Psi}_{p}\right\|_{1, \widetilde{\Omega}}\right) ;$

- $\left|\widetilde{b}_{h}\left(\underline{\Pi}_{1 h} \Psi_{p}, \widetilde{u}_{p}\right)-b\left(\widetilde{\Pi}_{1 h} \Psi_{p}, u_{p}\right)\right| \leq C h^{3}\left(\left\|\widetilde{u}_{p}\right\|_{3, \widetilde{\Omega}}+\left\|\widetilde{\Psi}_{p}\right\|_{1, \widetilde{\Omega}}\right)\left\|\widetilde{u}_{p}\right\|_{2, \tilde{\Omega}} ;$

- $\left|b_{h}^{\mathrm{NI}}\left(\underline{\Pi}_{1 h} \Psi_{p}, \mathcal{P}_{h} \widetilde{u}_{p}\right)-\widetilde{b}_{h}\left(\underline{\Pi}_{1 h} \Psi_{p}, \mathcal{P}_{h} \widetilde{u}_{p}\right)\right| \leq C h^{2}\left(\left\|\widetilde{u}_{p}\right\|_{3, \widetilde{\Omega}}+\left\|\widetilde{\Psi}_{p}\right\|_{1, \widetilde{\Omega}}\right)\left\|\widetilde{u}_{p}\right\|_{3, \widetilde{\Omega}} ;$

- $\left|\widetilde{b}_{h}\left(\widetilde{\Psi}_{p}, \mathcal{P}_{h} \widetilde{u}_{p}-\widetilde{u}_{p}\right)\right| \leq C h^{2}\left\|\widetilde{\Psi}_{p}\right\|_{1, \widetilde{\Omega}}\left\|\widetilde{u}_{p}\right\|_{3, \tilde{\Omega}} ;$

- $\lambda_{p}\left|\left\langle\widetilde{u}_{p}, \mathcal{P}_{h} \widetilde{u}_{p}-\widetilde{u}_{p}\right\rangle_{0, \Omega_{h}}\right| \leq C \lambda_{p} h^{3}\left\|\widetilde{u}_{p}\right\|_{3, \widetilde{\Omega}}^{2} ;$

- $\left|A\left(\Psi_{p}, \Psi_{p}\right)-\widetilde{A}_{h}\left(\widetilde{\Psi}_{p}, \widetilde{\Psi}_{p}\right)\right| \leq C h^{3}\left\|\widetilde{u}_{p}\right\|_{3, \widetilde{\Omega}}\left\|\widetilde{\Psi}_{p}\right\|_{1, \tilde{\Omega}}$

- $\left|A_{h}^{\mathrm{NI}}\left(\underline{\Pi}_{1 h} \Psi_{p}, \underline{\Pi}_{1 h} \Psi_{p}\right)-\widetilde{A}_{h}\left(\underline{\Pi}_{1 h} \Psi_{p}, \underline{\Pi}_{1 h} \Psi_{p}\right)\right| \leq C h^{2}\left(\left\|\widetilde{u}_{p}\right\|_{3, \widetilde{\Omega}}+\left\|\Psi_{p}\right\|_{1, \widetilde{\Omega}}\right)^{2}$;

- $\left|\widetilde{A}_{h}\left(\widetilde{\Psi}_{p}-\underline{\Pi}_{1 h} \Psi_{p}, \widetilde{\Psi}_{p}-\underline{\Pi}_{1 h} \Psi_{p}\right)\right| \leq C h^{2}\left(\left\|\widetilde{u}_{p}\right\|_{3, \widetilde{\Omega}}+\left\|\widetilde{\Psi}_{p}\right\|_{1, \widetilde{\Omega}}\right)^{2}$.

Then, from (4.65) and the estimates in (4.67)-(4.80), we get the required result: $\left|\lambda_{p}-\lambda_{p, h}\right|=O\left(h^{2}\right)$.

Theorem 4.5. Under the assumptions that Theorems 4.2, 4.3 and 4.4 hold, and $\lambda_{p}$ (resp. $\lambda_{p, h}$ ) is a simple eigenvalue of $\left(\mathbf{Q}^{\mathbf{E}}\right)$ (resp. $\left.\left(\mathbf{Q}_{\mathbf{h}}^{\mathbf{E}}\right)\right), 1 \leq p \leq N_{h}=\operatorname{dim} W_{h}$,

$$
\left\|\widetilde{\Psi}_{p}-\Psi_{p, h}\right\|_{0, \Omega_{h}}=O(h),\left\|\widetilde{u}_{p}-u_{p, h}\right\|_{1, \Omega_{h}}=O(h) .
$$


Proof. From (4.37) and (4.56), we have

$$
\begin{aligned}
\left\|\widetilde{\Psi}_{p}-\Psi_{p, h}\right\|_{0, \Omega_{h}} \leq & C h\left(\left[\left(\left\|\widetilde{u}_{p}\right\|_{3, \widetilde{\Omega}}+\left\|\widetilde{\Psi}_{p}\right\|_{1, \widetilde{\Omega}}\right)\left(1+\frac{2 \lambda_{p} \sqrt{2}}{\sqrt{\lambda_{1} \bar{\alpha}_{0}}}\left\{1+2 \frac{\lambda_{p}}{d_{p}}+(h+2)\left\|\widetilde{u}_{p}\right\|_{3, \widetilde{\Omega}}+h\left\|\widetilde{\Psi}_{p}\right\|_{1, \widetilde{\Omega}}\right\}\right)\right.\right. \\
& \left.\left.+\frac{2 \sqrt{2} \lambda_{p}}{\sqrt{\lambda_{1} \bar{\alpha}_{0}}} h^{2}\left\|\widetilde{u}_{p}\right\|_{1, \widetilde{\Omega}}^{2}\right]+\frac{\sqrt{2}}{\sqrt{\lambda_{1} \bar{\alpha}_{0}}} h\{\cdots\}\right)=O(h)
\end{aligned}
$$

where $\{\cdots\}$ denotes the expression within the curly brackets on the right-hand side of $(4.56)$. Putting $\chi_{h}=$ $\mathcal{P}_{h} \widetilde{u}_{p}$ in (4.1), we now find the estimates for the terms on the right-hand side of (4.1).

$$
\text { Indeed, from (4.9), }\left\|\widetilde{u}_{p}-\mathcal{P}_{h} \widetilde{u}_{p}\right\|_{1, \Omega_{h}} \leq C h^{2}\left\|\widetilde{u}_{p}\right\|_{3, \widetilde{\Omega}}
$$

Estimate for $\left\|\widetilde{\Psi}_{p}-\Psi_{p, h}\right\|_{0, \Omega_{h}}$ is obtained from (4.82). From Proposition 4.2, we have

$$
\begin{aligned}
\bullet \sup _{\Phi_{h} \in \mathbf{V}_{h}-\{0\}} \frac{\left|b_{h}^{\mathrm{NI}}\left(\Phi_{h}, \mathcal{P}_{h} \widetilde{u}_{p}\right)-\widetilde{b_{h}}\left(\Phi_{h}, \mathcal{P}_{h} \widetilde{u}_{p}\right)\right|}{\left\|\Phi_{h}\right\|_{1, \Omega_{h}}} & \leq \sup _{\Phi_{h} \in \mathbf{V}_{h}-\{0\}} \frac{C h^{2}\left\|\Phi_{h}\right\|_{1, \Omega_{h}}\left\|\mathcal{P}_{h} \widetilde{u}_{p}\right\|_{1, \Omega_{h}}}{\left\|\Phi_{h}\right\|_{1, \Omega_{h}}} \leq C h^{2}\left\|\widetilde{u}_{p}\right\|_{3, \widetilde{\Omega}} \\
\bullet \sup _{\Phi_{h} \in \mathbf{V}_{h}-\{0\}} \frac{\left|\tilde{A}_{h}\left(\widetilde{\Psi}_{p}, \Phi_{h}\right)-A\left(\Psi_{p}, \widetilde{\Phi}_{h}\right)\right|}{\left\|\Phi_{h}\right\|_{1, \Omega_{h}}} & \leq C h^{3}\left\|\widetilde{u}_{p}\right\|_{3, \widetilde{\Omega}} \\
\bullet \sup _{\Phi_{h} \in \mathbf{V}_{h}-\{0\}} \frac{\left|A_{h}^{\mathrm{NI}}\left(\Psi_{p, h}, \Phi_{h}\right)-\widetilde{A}_{h}\left(\Psi_{p, h}, \Phi_{h}\right)\right|}{\left\|\Phi_{h}\right\|_{1, \Omega_{h}}} & \leq \sup _{\Phi_{h} \in \mathbf{V}_{h}-\{0\}} \frac{C h^{2}\|A\|_{2, \infty, \widetilde{\Omega}}\left\|\Psi_{p, h}\right\|_{0, \Omega_{h}}\left\|\Phi_{h}\right\|_{0, \Omega_{h}}}{\left\|\Phi_{h}\right\|_{1, \Omega_{h}}} \text { (From } \\
& \leq C h^{2}\left(\left\|\widetilde{\Psi}_{p}-\Psi_{p, h}\right\|_{0, \Omega_{h}}+\left\|\widetilde{\Psi}_{p}\right\|_{1, \widetilde{\Omega}}\right)
\end{aligned}
$$

$$
\text { - } \sup _{\Phi_{h} \in \mathbf{V}_{h}-\{0\}} \frac{\left|\widetilde{b_{h}}\left(\Phi_{h}, \widetilde{u}_{p}\right)-b\left(\widetilde{\Phi}_{h}, u_{p}\right)\right|}{\left\|\Phi_{h}\right\|_{1, \Omega_{h}}} \leq \sup _{\Phi_{h} \in \mathbf{V}_{h}-\{0\}} \frac{C h^{3}\left\|\Phi_{h}\right\|_{1, \Omega_{h}}\left\|\widetilde{u}_{p}\right\|_{2, \widetilde{\Omega}}}{\left\|\Phi_{h}\right\|_{1, \Omega_{h}}} \leq C h^{3}\left\|\widetilde{u}_{p}\right\|_{2, \widetilde{\Omega}} \text {. }
$$

Substituting (4.82)-(4.87) in (4.1) with $\chi_{h}=\mathcal{P}_{h} \widetilde{u}_{p} \in W_{h}$, we have

$$
\begin{aligned}
\left\|\widetilde{u}_{p}-u_{p, h}\right\|_{1, \Omega_{h}} \leq & C h\left[h\left\|\widetilde{u}_{p}\right\|_{3, \widetilde{\Omega}}+h^{2}\left\|\widetilde{u}_{p}\right\|_{3, \widetilde{\Omega}}+(1+h)\left(\left[( \| \widetilde { u } _ { p } \| _ { 3 , \widetilde { \Omega } } + \| \widetilde { \Psi } _ { p } \| _ { 1 , \widetilde { \Omega } } ) \left(1+\frac{2 \lambda_{p} \sqrt{2}}{\sqrt{\lambda_{1} \bar{\alpha}_{0}}}\{1\right.\right.\right.\right. \\
& \left.\left.\left.\left.\left.+2 \frac{\lambda_{p}}{d_{p}}(h+2)\left\|\widetilde{u}_{p}\right\|_{3, \widetilde{\Omega}}+h\left\|\widetilde{\Psi}_{p}\right\|_{1, \widetilde{\Omega}}\right\}\right)+\frac{2 \sqrt{2} \lambda_{p}}{\sqrt{\lambda_{1} \bar{\alpha}_{0}}} h^{2}\left\|\widetilde{u}_{p}\right\|_{1, \widetilde{\Omega}}^{2}\right]+\frac{\sqrt{2}}{\sqrt{\lambda_{1} \bar{\alpha}_{0}}} h\{\ldots\}\right)+h\left\|\widetilde{\Psi}_{p}\right\|_{1, \widetilde{\Omega}}\right]
\end{aligned}
$$

where $\{\ldots\}$ denotes the expression within the curly brackets on the right-hand side of (4.56), from which the result follows.

Remark 5.3. As in the case of usual elliptic eigenvalue problems, the exponent of ' $h$ ' in (4.56) is optimal in the sense that it is twice the order of convergence for the corresponding source/steady state problem, i.e. for $\left\|\widetilde{u}-u_{h}\right\|_{1, \Omega_{h}}=O(h),\left\|\widetilde{\Psi}-\Psi_{h}\right\|_{0, \Omega_{h}}=O(h)$ of the corresponding source / steady state problem [10], $\left|\lambda_{p}-\lambda_{p, h}\right|=O\left(h^{2}\right)$.

Remark 5.4. In the case of eigenvalue problems, the estimates for simple eigenvalues and corresponding eigenelements: $\left|\lambda_{p}-\lambda_{p, h}\right|=O\left(h^{2}\right),\left\|\widetilde{u}_{p}-u_{p, h}\right\|_{1, \Omega_{h}}=O(h)$ and $\left\|\widetilde{\Psi}_{p}-\Psi_{p, h}\right\|_{0, \Omega_{h}}=O(h)$ have been obtained in (4.56) and (4.81) respectively under the assumptions that

(i) coefficients $A_{i j k l}$ have additional regularity (i.e. $A_{i j k l} \in W^{2, \infty}(\widetilde{\Omega}) \forall i, j, k, l=1,2$ ), and

(ii) the quadrature scheme (3.14) with $\mathrm{i}=1$ having higher algebraic degree of accuracy (i.e. exact for $P_{6}(\hat{T})$ ), 
has been used in the definition $(3.19)$ of $A_{h}^{\mathrm{NI}}(\cdot, \cdot)$, since the error estimates of the same order i.e. $\| \widetilde{u}-$ $u_{h}\left\|_{1, \Omega_{h}}=O(h),\right\| \widetilde{\Psi}-\Psi_{h} \|_{0, \Omega_{h}}=O(h)$ have been obtained in [10] for the corresponding source problem for (i') $A_{i j k l} \in W^{1, \infty}(\widetilde{\Omega}) \quad \forall i, j, k, l=1,2$ and (i'i') the quadrature scheme (3.14) with $\mathrm{i}=1$ exact for $P_{4}(\hat{T})$, which has been used in the definition $(3.19)$ of $A_{h}^{\mathrm{NI}}(\cdot, \cdot)$ (see Th. 5.2 of [10] ).

But these estimates $\left\|\widetilde{u}_{p}-u_{p, h} \downarrow_{1, \Omega_{h}}=O(h),\right\| \widetilde{\Psi}_{p}-\Psi_{p, h} \|_{0, \Omega_{h}}=O(h)$ in (4.81) for the eigenvalue problem (resp. $\left\|\widetilde{u}-u_{h}\right\|_{1, \Omega_{h}}=O(h),\left\|\Psi-\Psi_{h}\right\|_{0, \Omega_{h}}=O(h)$ for the corresponding source problem in [10]) cannot be improved upon by assuming still more regularity i.e. $A_{i j k l} \in W^{m, \infty}(\widetilde{\Omega})$ with $m>2$ (resp. $W^{m, \infty}(\widetilde{\Omega})$ with $m>1)$ and using quadrature scheme $(3.14)$ with $\mathrm{i}=1$ exact for $P_{m}(\hat{T})$ with $m>6\left(\operatorname{resp} . P_{m}(\hat{T})\right.$ with $\left.m>4\right)$ in the definition (3.19) of $A_{h}^{\mathrm{NI}}(\cdot, \cdot)$.

\section{NUMERICAL EXAMPLES}

In this section, we would consider numerical examples on eigenvalue problems defined in (2.1), the coefficients $a_{i j k l}$ for which satisfy (A1-A2). The convex domains $\Omega$ with curved boundary considered are approximated by a polygonal boundary $\Gamma_{h}^{\mathrm{pol}}$ and a curved boundary $\Gamma_{h}$ constructed with the help of an isoparametric mapping. The fundamental and a few higher frequencies and mode shapes of a class of orthotropic plates with clamped boundary conditions are computed and the results obtained are compared with the existing results.

For the plate bending operator $\Lambda$, the eigenvalue problem (2.1) is obtained from the equation of motion for the small transverse displacement $U U\left(x_{1}, x_{2} ; t\right)$ of the vibrating elastic plate under consideration:

$$
\left.\left.\Lambda U+\rho \frac{\partial^{2} U}{\partial t^{2}}=0 \quad \forall\left(\left(x_{1}, x_{2}\right) ; t\right) \in \Omega \times\right] 0, T\right]
$$

with $\left.\left.\left.U\right|_{\Gamma}=0,\left.\frac{\partial U}{\partial n}\right|_{\Gamma}=0 \quad \forall t \in\right] 0, T\right], \rho$ being the mass density of the elastic plate per unit area measure of $\bar{\Omega}$, when free natural vibrations are assumed and the motion is defined by:

$$
U\left(x_{1}, x_{2} ; t\right)=u\left(x_{1}, x_{2}\right) \cos \omega t
$$

$\omega$ being the circular frequency expressed in radians/unit time, i.e. a substitution of (5.2) into (5.1) will yield (2.1) with $\lambda=\rho \omega^{2}$.

In the practical applications (examples considered below), dimensionless coordinates are introduced and instead of $\lambda=\rho \omega^{2}$, some new parameter of convenience which will depend on $\rho, \omega$, characteristic plate size parameter, flexural rigidity of the plate etc will be introduced and will still be denoted by the same notation $\lambda$ by giving its new definition without deduction,for which we refer to [26].

- For constant coefficients $A_{i j k l}$ (or equivalently $a_{i j k l}$ ), which will be considered in the examples, introducing suitable canonical bases $\left\{\Phi_{h}^{i}\right\}_{i=1}^{3 N_{1}}$ in $\mathbf{V}_{h}$ and $\left\{\chi_{h}^{i}\right\}_{i=1}^{N_{0}}$ in $W_{h}$, the isoparametric mixed finite element eigenvalue problem $\left(\mathbf{Q}_{\mathbf{h}}^{\mathbf{E}}\right)$ can be reduced to the following problem in matrix form (see [31] for details):

Find $\left(\lambda_{h} ;(\underline{\alpha} ; \underline{\beta})\right)=\left(\lambda_{h} ;\left(\underline{\alpha}^{1}, \underline{\alpha}^{2}, \underline{\alpha}^{3}, \underline{\beta}\right)\right) \in \mathbb{R}^{+} \times \mathbb{R}^{3 N_{1}+N_{0}}$ such that

$$
\begin{aligned}
c_{11}[A] \underline{\alpha}^{1}+c_{12}[A] \underline{\alpha}^{2}+c_{13}[A] \underline{\alpha}^{3}+\left[B_{1}\right] \underline{\beta} & =\mathbf{0} \\
c_{12}[A] \underline{\alpha}^{1}+c_{22}[A] \underline{\alpha}^{2}+c_{23}[A] \underline{\alpha}^{3}+\left[B_{2}\right] \underline{\beta} & =\mathbf{0} \\
c_{13}[A] \underline{\alpha}^{1}+c_{23}[A] \underline{\alpha}^{2}+c_{33}[A] \underline{\alpha}^{3}+\left[B_{3}\right] \underline{\beta} & =\mathbf{0} \\
{\left[B_{1}\right]^{t} \underline{\alpha}^{1}+\left[B_{2}\right]^{t} \underline{\alpha}^{2}+\left[B_{3}\right]^{t} \underline{\alpha}^{3} } & =\lambda_{h}[M] \underline{\beta}
\end{aligned}
$$


where $[A]_{N_{1} \times N_{1}}$ is a symmetric, positive-definite matrix of order $N_{1}[31] ; c_{i j} \in \mathbb{R}$ with $c_{12}=0, \quad c_{23}=0$ for the class of Orthotropic Plates considered in the examples,

$\underline{\alpha}^{i} \in \mathbb{R}^{N_{1}}, \underline{\beta} \in \mathbb{R}^{N_{0}} \quad$ such $\quad$ that $\quad \Psi_{h}=\sum_{j=1}^{3 N_{1}} \alpha_{j} \Phi_{h}^{j}, u_{h}=\sum_{j=1}^{N_{0}} \beta_{j} \chi_{h}^{j} \quad$ with $\quad \underline{\alpha}^{1}=\left(\alpha_{j}\right)_{j=1}^{N_{1}}, \quad \underline{\alpha}^{2}=\left(\alpha_{j}\right)_{j=N_{1}+1}^{2 N_{1}}$, $\underline{\alpha}^{3}=\left(\alpha_{j}\right)_{j=2 N_{1}+1}^{3 N_{1}}, \quad \underline{\beta}=\left(\beta_{i}\right)_{i=1}^{N_{0}}, \quad\left[B_{i}\right]_{N_{1} \times N_{0}}$ is a rectangular matrix of size $N_{1} \times N_{0}$ with its transpose denoted by $\left[B_{i}\right]^{t} ;[M]_{N_{0} \times N_{0}}$ is the symmetric, positive-definite global mass matrix of order $N_{0} \times N_{0}$ got after assembling the element mass matrices $\left[M_{T}\right]$.

Then, $\underline{\alpha}^{1}, \underline{\alpha}^{2}, \underline{\alpha}^{3}$ can be eliminated from the first, second and third equations in (5.3) and substituting the expressions for $\underline{\alpha}^{i}$ in the fourth equation in (5.3), we get

$$
[K]_{N_{0} \times N_{0}} \quad \underline{\beta}=\lambda_{h}[M]_{N_{0} \times N_{0}} \underline{\beta}
$$

where $[K]$ is the symmetric, positive-definite, global stiffness matrix of order $N_{0} \times N_{0}$.

Solving (5.4) for $\left(\lambda_{h} ; \underline{\beta}\right)$, we can find $\underline{\alpha}^{i}$ using the expression used in the elimination of $\underline{\alpha}^{i}, \quad 1 \leq i \leq 3$.

- (5.4) has been solved by Subspace Iteration Method, although Lanczos method can also be efficiently used.

Example: Clamped Orthotropic Elliptic Plate Problem. The coefficients $a_{i j k l}$ for the orthotropic case are:

$$
\begin{aligned}
& a_{i i i i}=D_{i i} ; a_{1122}=a_{2211}=D_{12}=\nu_{1} D_{22}=\nu_{2} D_{11} ; \\
& a_{1212}=a_{2121}=a_{2112}=a_{1221}=D_{t}, a_{i j k l}=0 \text { otherwise }
\end{aligned}
$$

$$
\text { where } \begin{aligned}
D_{i i} & =\frac{E_{i} t^{3}}{12\left(1-\nu_{1} \nu_{2}\right)}(i=1,2) \\
D_{t} & =\frac{G h^{3}}{12}>0, \quad H=\nu_{2} D_{1}+2 D_{t} \\
G & =\frac{E_{1} E_{2}}{E_{1}+\left(1+2 \nu_{1}\right) E_{2}}>0, E_{1} \nu_{2}=E_{2} \nu_{1},
\end{aligned}
$$

$E_{i}$ and $\nu_{i}$ are Young's moduli and Poisson's coefficients respectively, $t\left(x_{1}, x_{2}\right)$ being the thickness function.

We consider the following cases where the Poisson's coefficient $\nu_{1}=1 / 3$ and the flexural rigidities are given by:

Case I: $\quad D_{12} / D_{22}=1 / 3, D_{11} / D_{22}=1$

Case II: $D_{12} / D_{22}=1 / 3, D_{11} / D_{22}=1 / 3$

Case III: $D_{12} / D_{22}=1, \quad D_{11} / D_{22}=1$

Case IV: $D_{12} / D_{22}=1, \quad D_{11} / D_{22}=1 / 3$.

The eigenvalue problem (2.1) with $\lambda^{2}=\omega \mathbf{a}^{2} \sqrt{\rho \mathbf{t} / \mathbf{D}_{22}}$ corresponding to the natural vibrations of the clamped orthotropic elliptic plate with $b / a=0.5$, ' $a$ ' being the semi-major axis and ' $b$ ' being the semi-minor axis is considered. The first few eigenvalues and the corresponding eigenvectors for both polygonal and curved approximations have been computed and only the eigenvalues, which are compared with those given in [19], are shown in Tables I-IV below. 
TABle 1. Case I: $D_{12} / D_{22}=1 / 3, D_{11} / D_{22}=1, \nu=1 / 3, b / a=0.5$.

\begin{tabular}{|c|c|c|c|c|c|}
\hline Eigenvalues & $\begin{array}{c}\text { Polygonal } \\
\text { Approximation }\end{array}$ & $\begin{array}{c}\text { Isoparametric } \\
\text { Approximation }\end{array}$ & [19] & $\begin{array}{c}\text { Nature of the } \\
\text { Mode }\end{array}$ & $\begin{array}{c}\text { Nodal } \\
\text { Pattern }\end{array}$ \\
\hline$\lambda_{1, \mathrm{~h}}$ & 27.90 & 27.41 & 27.38 & $\begin{array}{c}\text { Doubly } \\
\text { Symmetric }\end{array}$ & \\
\hline$\lambda_{2, \mathrm{~h}}$ & 40.45 & 39.68 & 39.49 & $\begin{array}{c}\text { Symmetric } \\
\text { Antisymmetric }\end{array}$ & \\
\hline$\lambda_{3, \mathrm{~h}}$ & 57.73 & 56.55 & 55.97 & $\begin{array}{c}\text { Second Doubly } \\
\text { Symmetric }\end{array}$ & \\
\hline$\lambda_{4, \mathrm{~h}}$ & 71.32 & 70.07 & 69.87 & $\begin{array}{c}\text { Antisymmetric } \\
\text { Symmetric }\end{array}$ & \\
\hline$\lambda_{5, \mathrm{~h}}$ & 80.00 & 78.25 & 76.99 & $\begin{array}{c}\text { Second Symmetric } \\
\text { Antisymmetric }\end{array}$ & \\
\hline
\end{tabular}

TABle 2. Case II: $D_{12} / D_{22}=1 / 3, D_{11} / D_{22}=1 / 3, \nu=1 / 3, b / a=0.5$.

\begin{tabular}{|c|c|c|c|c|c|}
\hline Eigenvalues & $\begin{array}{c}\text { Polygonal } \\
\text { Approximation }\end{array}$ & $\begin{array}{c}\text { Isoparametric } \\
\text { Approximation }\end{array}$ & /19/ & $\begin{array}{c}\text { Nature of the } \\
\text { Mode }\end{array}$ & $\begin{array}{c}\text { Nodal } \\
\text { Pattern }\end{array}$ \\
\hline$\lambda_{1, \mathrm{~h}}$ & 27.24 & 26.76 & 26.73 & $\begin{array}{c}\text { Doubly } \\
\text { Symmetric }\end{array}$ & \\
\hline$\lambda_{2, \mathrm{~h}}$ & 37.33 & 36.64 & 36.43 & $\begin{array}{c}\text { Symmetric } \\
\text { Antisymmetric }\end{array}$ & \\
\hline$\lambda_{3, \mathrm{~h}}$ & 49.78 & 48.82 & 48.23 & $\begin{array}{c}\text { Second Doubly } \\
\text { Symmetric }\end{array}$ & \\
\hline$\lambda_{4, \mathrm{~h}}$ & 64.91 & 63.55 & 62.30 & $\begin{array}{c}\text { Second Symmetric } \\
\text { Antisymmetric }\end{array}$ & \\
\hline$\lambda_{5, \mathrm{~h}}$ & 70.81 & 69.59 & 69.38 & $\begin{array}{c}\text { Antisymmetric } \\
\text { Symmetric }\end{array}$ & \\
\hline
\end{tabular}

TABle 3 . Case III: $D_{12} / D_{22}=1, D_{11} / D_{22}=1, \nu=1 / 3, b / a=0.5$.

\begin{tabular}{|c|c|c|c|c|c|}
\hline Eigenvalues & $\begin{array}{c}\text { Polygonal } \\
\text { Approximation }\end{array}$ & $\begin{array}{c}\text { Isoparametric } \\
\text { Approximation }\end{array}$ & [19] & $\begin{array}{c}\text { Nature of the } \\
\text { Mode }\end{array}$ & $\begin{array}{c}\text { Nodal } \\
\text { Pattern }\end{array}$ \\
\hline$\lambda_{1, \mathrm{~h}}$ & 30.46 & 29.93 & 29.88 & $\begin{array}{c}\text { Doubly } \\
\text { Symmetric }\end{array}$ & \\
\hline$\lambda_{2, \mathrm{~h}}$ & 47.45 & 46.56 & 46.34 & $\begin{array}{c}\text { Symmetric } \\
\text { Antisymmetric }\end{array}$ & \\
\hline$\lambda_{3, \mathrm{~h}}$ & 68.04 & 66.73 & 66.09 & $\begin{array}{c}\text { Second Doubly } \\
\text { Symmetric }\end{array}$ & \\
\hline$\lambda_{4, \mathrm{~h}}$ & 76.10 & 74.77 & 74.56 & $\begin{array}{c}\text { Antisymmetric } \\
\text { Symmetric }\end{array}$ & \\
\hline$\lambda_{5, \mathrm{~h}}$ & 93.11 & 91.19 & 89.73 & $\begin{array}{c}\text { Second Symmetric } \\
\text { Antisymmetric }\end{array}$ & \\
\hline
\end{tabular}


TABLE 4. Case IV: $D_{12} / D_{22}=1, D_{11} / D_{22}=1 / 3, \nu=1 / 3, b / a=0.5$.

\begin{tabular}{|c|c|c|c|c|c|}
\hline Eigenvalues & $\begin{array}{c}\text { Polygonal } \\
\text { Approximation }\end{array}$ & $\begin{array}{l}\text { Isoparametric } \\
\text { Approximation }\end{array}$ & {$[19]$} & $\begin{array}{l}\text { Nature of the } \\
\text { Mode }\end{array}$ & $\begin{array}{c}\text { Nodal } \\
\text { Pattern }\end{array}$ \\
\hline$\lambda_{1, \mathrm{~h}}$ & 29.93 & 29.41 & 29.38 & $\begin{array}{c}\text { Doubly } \\
\text { Symmetric }\end{array}$ & \\
\hline$\lambda_{2, \mathrm{~h}}$ & 44.91 & 44.09 & 43.87 & $\begin{array}{c}\text { Symmetric } \\
\text { Antisymmetric }\end{array}$ & \\
\hline$\lambda_{3, \mathrm{~h}}$ & 61.43 & 60.29 & 59.68 & $\begin{array}{l}\text { Second Doubly } \\
\text { Symmetric }\end{array}$ & \\
\hline$\lambda_{4, \mathrm{~h}}$ & 75.74 & 74.44 & 74.24 & $\begin{array}{c}\text { Antisymmetric } \\
\text { Symmetric }\end{array}$ & \\
\hline$\lambda_{5, \mathrm{~h}}$ & 80.37 & 78.74 & 77.29 & $\begin{array}{l}\text { Second Symmetric } \\
\text { Antisymmetric }\end{array}$ & \\
\hline
\end{tabular}

Acknowledgements. The first author expresses his heartiest thanks and deep sense of gratitude to Professor Olivier Pironneau, whose excellent hospitality and full academic support contributed to the speedy completion of a major part of the present work during his stay in the Laboratory of Numerical Analysis, C.N.R.S., Université Pierre et Marie Curie, Paris VI, and for providing financial support for this visit, he expresses special thanks to the French Ministry of External Affairs.

\section{REFERENCES}

[1] R.A. Adams, Sobolev Spaces. Academic Press, New York (1975).

[2] I. Babuška and J.E. Osborn, Eigenvalue Problems, in Handbook of Numerical Analysis. Vol. II, P.G. Ciarlet and J.-L. Lions, Eds., in Finite Element Methods (Part 1), North-Holland Publishing Company, Amsterdam, New York, Oxford (1991) 641-787.

[3] S. Balasundaram and P.K. Bhattacharyya, On existence of solution of the Dirichlet problem of fourth-order partial differential equations with variable coefficients. Quart. Appl. Math. 39 (1983) 311-317.

[4] S. Balasundaram and P.K. Bhattacharyya, A mixed finite element method for fourth-order elliptic equations with variable coefficients. Comput. Math. Appl. 10 (1984) 245-256.

[5] U. Banerjee, A note on the effect of numerical quadrature in finite element eigenvalue approximation. Numer. Math. 61 (1992) $145-152$.

[6] U. Banerjee and J.E. Osborn, Estimates of the effect of numerical integration in finite element eigenvalue approximation. Numer. Math. 56 (1990) 735-762.

[7] M. Bernadou, Méthodes numériques pour les problèmes elliptiques, in Méthodes de Mathématiques Appliquées, Vol. 1, Chap. XI, C.E.A., France (1981).

[8] P.K. Bhattacharyya and N. Nataraj, Isoparametric Mixed Finite Element Approximation of Eigenvalues and Eigenvectors of 4th-Order Eigenvalue Problems with Variable Coefficients. Research Report No. R 98021, Laboratoire d' Analyse Numérique, Université de Paris VI (1998).

[9] P.K. Bhattacharyya and N. Nataraj, On Combined Effect of Boundary Approximation and Numerical Integration on Mixed Finite Element Solution of 4th order Elliptic Problems with Variable Coefficients. ESAIM: M2AN 133 (1999) 807-836.

[10] P.K. Bhattacharyya and N. Nataraj, Error Estimates for Isoparametric Mixed Finite Element Solution of 4th-Order Elliptic Problems with Variable Coefficients. In preparation.

[11] F. Brezzi, On the existence, uniqueness and approximation of saddle-point problems arising from Lagrangian multipliers. RAIRO Anal. Numér. 8 (1974) 129-151. 
[12] F. Brezzi and M. Fortin, Mixed and Hybrid Finite Element Methods. Springer-Verlag, Berlin, Heidelberg, New York (1991).

[13] F. Brezzi and P.A. Raviart, Mixed finite element methods for 4th-order elliptic equations. Topics Numer. Anal. III, J. Miller, Ed., Academic Press, New York (1978) 33-56.

[14] C. Canuto, Eigenvalue approximation by mixed methods. RAIRO Anal. Numér. 12 (1978) 27-50.

[15] F. Chatelin, Spectral Approximation of Linear Operators. Academic Press, New York (1983).

[16] P.G. Ciarlet, The Finite Element Method for Elliptic Problems. North-Holland Publishing Company, Amsterdam, New York, Oxford (1978).

[17] P.G. Ciarlet and P.A. Raviart, The combined effect of curved boundaries and numerical integration in isoparametric finite element methods, in Math. Found. Finite El. Method Appl. Part. Differ. Equations, A.K. Aziz and I. Babuška, Eds., Sympos. Univ. Maryland, Baltimore (1972) 409-474.

[18] M. Dauge, Elliptic Boundary Value Problems on Corner Domains. Springer-Verlag, Berlin, Heidelberg, New York (1988).

[19] N.J. DeCapua and B.C. Sun, Transverse vibration of a class of orthotropic plates. J. Appl. Mech. (1972) 613-615.

[20] S. Gopalsamy and P.K. Bhattacharyya, On existence and uniqueness of solution of boundary value problems of fourth-order elliptic partial differential equations with variable coefficients. J. Math. Anal. Appl. 136 (1988) 589-608.

[21] P. Grisvard, Elliptic Problems in Nonsmooth Domains. Pitman, Boston (1985).

[22] K. Ishihara, A mixed finite element method for the biharmonic eigenvalue problem of plate bending. Publ. Res. Inst. Math. Sci. Kyoto Univ. 14 (1978) 399-414.

[23] K. Ishihara, The buckling of plates by the mixed finite element method. Mem. Numer. Math. 5 (1978) 73-82.

[24] V.A. Kondratev, Boundary value problems for elliptic equations in domains with conical or angular points. Trudy Moskov. Mat. Obshch. 16 (1967) 209-292.

[25] M.P. Lebaud, Error estimate in an isoparametric finite element Eigenvalue Problem. Math. Comp. 63 (1994) 19-40.

[26] A.W. Leissa, Vibration of Plates. NASA SP-160 (1969).

[27] S.G. Leknitskii, Anisotropic Plates. Gordon and Breach Science Publishers, New York (1968).

[28] J.-L. Lions, Problèmes aux limites dans les équations aux dérivées partielles. Presses Univ. Montréal, Montreal (1965).

[29] B. Mercier, J. Osborn, J. Rappaz and P.A. Raviart, Eigenvalue approximation by mixed and hybrid methods. Math. Comp. 36 (1981) 427-453.

[30] T. Miyoshi, A finite element method for the solution of fourth-order partial differential equations. Kumamoto J. Sci. (Math.) 9 (1973) 87-116.

[31] N. Nataraj, On Mixed Finite Element Analysis Of Fourth Order Elliptic Source/Eigenvalue Problems in Convex Domains. Ph.D. Thesis, Dept. of Mathematics, Indian Institute of Technology, Delhi (1998).

[32] L.A. Oganesian and L.A. Rukhovec, Variational difference methods for the solution of elliptic problems. Izv. Akad. Nauk Armyan. SSR, Yerevan (1979). In Russian.

[33] A.B. Ouaritini, Méthodes d'éléments finis mixtes pour des problèmes de coques minces. Thèse de Docteur de $3^{e}$ cycle, Université de Pau et des Pays de L'Adour, France (1984).

[34] O. Pironneau, Méthodes des éléments finis pour les fluides. Masson, Paris (1988).

[35] P.A. Raviart and J.M. Thomas, Introduction à l'analyse numérique des équations aux dérivées partielles. Masson, Paris (1983).

[36] J.E. Roberts and J.M. Thomas, Mixed and Hybrid Methods, in Handbook of Numerical Analysis. Vol. II, in Finite Element Methods (Part 1), P.G. Ciarlet and J.-L. Lions, Eds., North-Holland Publishing Company, Amsterdam, New York, Oxford, (1991) 523-633.

[37] G. Strang and G.J. Fix, An Analysis of the Finite Element Method. Prentice-Hall, New York (1973).

[38] S. Timoshenko and S. Woinowsky-Kreiger, Theory of Plates and Shells. McGraw-Hill Book Company, New York (1959).

[39] M. Vanmaele and A. Ženíšek, External finite-element approximations of eigenvalue problems. RAIRO Modél. Math. Anal. Numér. 27 (1993) 565-589.

[40] M. Vanmaele and A. Ženíšek, The combined effect of numerical integration and approximation of boundary in the finite element method for eigenvalue problems. Numer. Math. (1995).

[41] A. Zeníšek, Nonlinear Elliptic and Evolution Problems and their Finite Element Approximations. Academic Press, New York (1990).

To access this journal online:

www.edpsciences.org 